\title{
Constraints on the off-shell Higgs boson signal strength in the high-mass $Z Z$ and $W W$ final states with the ATLAS detector
}

\author{
ATLAS Collaboration ${ }^{\star}$ \\ CERN, 1211 Geneva 23, Switzerland
}

Received: 4 March 2015 / Accepted: 29 June 2015 / Published online: 17 July 2015

(C) CERN for the benefit of the ATLAS collaboration 2015. This article is published with open access at Springerlink.com

\begin{abstract}
Measurements of the $Z Z$ and $W W$ final states in the mass range above the $2 m_{Z}$ and $2 m_{W}$ thresholds provide a unique opportunity to measure the off-shell coupling strength of the Higgs boson. This paper presents constraints on the off-shell Higgs boson event yields normalised to the Standard Model prediction (signal strength) in the $Z Z \rightarrow 4 \ell$, $Z Z \rightarrow 2 \ell 2 \nu$ and $W W \rightarrow e v \mu \nu$ final states. The result is based on $p p$ collision data collected by the ATLAS experiment at the LHC, corresponding to an integrated luminosity of $20.3 \mathrm{fb}^{-1}$ at a collision energy of $\sqrt{s}=8 \mathrm{TeV}$. Using the $C L_{s}$ method, the observed $95 \%$ confidence level (CL) upper limit on the off-shell signal strength is in the range 5.1-8.6, with an expected range of 6.7-11.0. In each case the range is determined by varying the unknown $g g \rightarrow Z Z$ and $g g \rightarrow W W$ background K-factor from higher-order quantum chromodynamics corrections between half and twice the value of the known signal K-factor. Assuming the relevant Higgs boson couplings are independent of the energy scale of the Higgs boson production, a combination with the on-shell measurements yields an observed (expected) $95 \%$ CL upper limit on $\Gamma_{H} / \Gamma_{H}^{\mathrm{SM}}$ in the range 4.5-7.5 (6.5-11.2) using the same variations of the background $\mathrm{K}$-factor. Assuming that the unknown $g g \rightarrow V V$ background $\mathrm{K}$-factor is equal to the signal $\mathrm{K}$-factor, this translates into an observed (expected) $95 \%$ CL upper limit on the Higgs boson total width of 22.7 (33.0) $\mathrm{MeV}$.
\end{abstract}

\section{Introduction}

The observation of a new particle in the search for the Standard Model (SM) Higgs boson at the LHC, reported by the ATLAS [1] and CMS [2] Collaborations, is a milestone in the quest to understand electroweak symmetry breaking. Precision measurements of the properties of the new boson are of critical importance. Among its key properties are the couplings to each of the SM fermions and bosons, for which ATLAS and CMS presented results in Refs. [3,4],

^e-mail: atlas.publications@ @ern.ch and spin/CP properties, studied by ATLAS and CMS in Refs. [5,6].

The studies in Refs. [7-10] have shown that the high-mass off-peak regions beyond $2 m_{V}(V=Z, W)$, well above the measured resonance mass of about $125 \mathrm{GeV}$ [4,11], in the $H \rightarrow Z Z$ and $H \rightarrow W W$ channels are sensitive to Higgs boson production through off-shell and background interference effects. This presents a novel way of characterising the properties of the Higgs boson in terms of the off-shell event yields, normalised to the SM prediction (referred to as signal strength $\mu$ ), and the associated off-shell Higgs boson couplings. Such studies provide sensitivity to new physics that alters the interactions between the Higgs boson and other fundamental particles in the high-mass region [12-18]. This approach was used by the CMS Collaboration [19] to set an indirect limit on the Higgs boson total width. The analysis presented in this paper is complementary to direct searches for Higgs boson to invisible decays [20,21] and to constraints coming from the Higgs boson coupling tests [3,4].

This paper presents an analysis of the off-shell signal strength in the $Z Z \rightarrow 4 \ell, Z Z \rightarrow 2 \ell 2 v$ and $W W \rightarrow e v \mu \nu$ final states $(\ell=e, \mu)$. It is structured as follows: Sect. 2 discusses the key theoretical considerations and the simulation of the main signal and background processes. Sections 3-5 give details for the analysis in the $Z Z \rightarrow 4 \ell, Z Z \rightarrow 2 \ell 2 \nu$ and $W W \rightarrow e v \mu \nu$ final states, respectively. The dominant systematic uncertainties are discussed in Sect. 6. Finally the results of the individual analyses and their combination are presented in Sect. 7.

The ATLAS detector is described in Ref. [22]. The present analysis is performed on $p p$ collision data corresponding to an integrated luminosity of $20.3 \mathrm{fb}^{-1}$ at a collision energy of $\sqrt{s}=8 \mathrm{TeV}$.

\section{Theoretical predictions and simulated samples}

The cross-section $\sigma_{\text {off-shell }}^{g g \rightarrow H^{*}} \rightarrow V V$ for the off-shell Higgs boson production through gluon fusion with subsequent decay into 


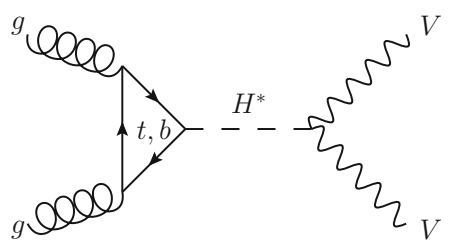

(a)

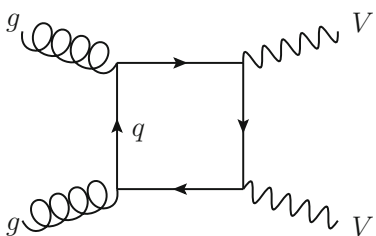

(b)

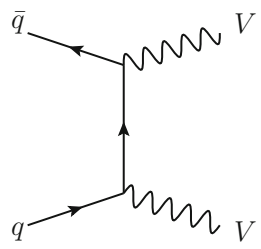

(c)

Fig. 1 The leading-order Feynman diagrams for a the $g g \rightarrow H^{*} \rightarrow V V$ signal, b the continuum $g g \rightarrow V V$ background and $\mathbf{c}$ the $q \bar{q} \rightarrow V V$ background

vector-boson pairs, ${ }^{1}$ as illustrated by the Feynman diagram in Fig. 1a, is proportional to the product of the Higgs boson couplings squared for production and decay. However, unlike the on-shell Higgs boson production, $\sigma_{\text {off-shell }}^{g g \rightarrow H^{*}} \rightarrow V V$ is independent of the total Higgs boson decay width $\Gamma_{H}[7,8]$. Using the framework for Higgs boson coupling deviations as described in Ref. [23], the off-shell signal strength in the high-mass region selected by the analysis described in this paper at an energy scale $\hat{s}, \mu_{\text {off-shell }}(\hat{s})$, can be expressed as:

$\mu_{\text {off-shell }}(\hat{s}) \equiv \frac{\sigma_{\text {off-shell }}^{g g \rightarrow H^{*} \rightarrow V V}(\hat{s})}{\sigma_{\text {off-shell, SM }}^{g g \rightarrow H^{*} \rightarrow V V}(\hat{s})}=\kappa_{g, \text { off-shell }}^{2}(\hat{s}) \cdot \kappa_{V, \text { off-shell }}^{2}(\hat{s})$,

where $\kappa_{g \text {,off-shell }}(\hat{s})$ and $\kappa_{V \text {,off-shell }}(\hat{s})$ are the off-shell coupling scale factors associated with the $g g \rightarrow H^{*}$ production and the $H^{*} \rightarrow V V$ decay. Due to the statistically limited sensitivity of the current analysis, the off-shell signal strength and coupling scale factors are assumed in the following to be independent of $\hat{s}$ in the high-mass region selected by the analysis. The off-shell Higgs boson signal cannot be treated independently from the $g g \rightarrow V V$ background, as sizeable negative interference effects appear [7]. The interference term is proportional to $\sqrt{\mu_{\text {off-shell }}}=\kappa_{g, \text { off-shell }} \cdot \kappa_{V \text {,off-shell }}$.

In contrast, the cross-section for on-shell Higgs boson production allows a measurement of the signal strength:

$\mu_{\text {on-shell }} \equiv \frac{\sigma_{\text {on-shell }}^{g g \rightarrow H \rightarrow V V}}{\sigma_{\text {on-shell, SM }}^{g g \rightarrow H \rightarrow V V}}=\frac{\kappa_{g, \text { on-shell }}^{2} \cdot \kappa_{V, \text { on-shell }}^{2}}{\Gamma_{H} / \Gamma_{H}^{\mathrm{SM}}}$,

which depends on the total width $\Gamma_{H}$. Assuming identical on-shell and off-shell Higgs boson coupling scale factors, the ratio of $\mu_{\text {off-shell }}$ to $\mu_{\text {on-shell provides a measurement of }}$ the total width of the Higgs boson. This assumption is particularly relevant to the running of the effective coupling $\kappa_{g}(\hat{s})$ for the loop-induced $g g \rightarrow H$ production process, as it is

\footnotetext{
${ }^{1}$ In the following the notation $g g \rightarrow\left(H^{*} \rightarrow\right) V V$ is used for the full signal + background process for $V V=Z Z$ and $W W$ production, including the Higgs boson signal (S) $g g \rightarrow H^{*} \rightarrow V V$ process, the continuum background (B) $g g \rightarrow V V$ process and their interference. For vector-boson fusion (VBF) production, the analogous notation VBF $\left(H^{*} \rightarrow\right) V V$ is used for the full signal plus background process, with VBF $H^{*} \rightarrow V V$ representing the Higgs boson signal and VBF $V V$ denoting the background.
}

sensitive to new physics that enters at higher mass scales and could be probed in the high-mass $m_{V V}$ signal region of this analysis. More details are given in Refs. [12-16]. With the current sensitivity of the analysis, only an upper limit on the total width $\Gamma_{H}$ can be determined, for which the weaker assumption

$\kappa_{g, \text { on-shell }}^{2} \cdot \kappa_{V, \text { on-shell }}^{2} \leq \kappa_{g, \text { off-shell }}^{2} \cdot \kappa_{V, \text { off-shell }}^{2}$,

that the on-shell couplings are no larger than the off-shell couplings, is sufficient. It is also assumed that any new physics which modifies the off-shell signal strength $\mu_{\text {off-shell }}$ and the off-shell couplings $\kappa_{i}$,off-shell does not modify the predictions for the backgrounds. Further, neither are there sizeable kinematic modifications to the off-shell signal nor new, sizeable signals in the search region of this analysis unrelated to an enhanced off-shell signal strength [18,24].

While higher-order quantum chromodynamics (QCD) and electroweak (EW) corrections are known for the off-shell signal process $g g \rightarrow H^{*} \rightarrow Z Z$ [25], which are also applicable to $g g \rightarrow H^{*} \rightarrow W W$, no higher-order QCD calculations are available for the $g g \rightarrow V V$ background process, which is evaluated at leading order (LO). Therefore the results are given as a function of the unknown $\mathrm{K}$-factor for the $g g \rightarrow V V$ background. QCD corrections for the off-shell signal processes have only been calculated inclusively in the jet multiplicity. The experimental analyses are therefore performed inclusively in jet observables, and the event selections are designed to minimise the dependence on the boost of the $V V$ system, which is sensitive to the jet multiplicity.

The dominant processes contributing to the high-mass signal region in the $Z Z \rightarrow 4 \ell, Z Z \rightarrow 2 \ell 2 v$ and $W W \rightarrow e v \mu \nu$ final states are: the $g g \rightarrow H^{*} \rightarrow V V$ off-shell signal, the $g g \rightarrow V V$ continuum background, the interference between them, $V V$ production in association with two jets through $\mathrm{VBF}$ and $V H$-like production modes $p p \rightarrow V V+2 j(s-$, $t$ - and $u$-channel) and the $q \bar{q} \rightarrow V V$ background. The LO Feynman diagrams for the $g g \rightarrow H^{*} \rightarrow V V$ signal, the continuum $g g \rightarrow V V$ background and the dominant irreducible $q \bar{q} \rightarrow V V$ background are depicted in Fig. 1 . The $W W \rightarrow e v \mu \nu$ channel also receives sizeable background contributions from $t \bar{t}$ and single-top production. In the following a Higgs boson mass of $m_{H}=125.5 \mathrm{GeV}$, close to the ATLAS-measured Higgs boson mass value of 


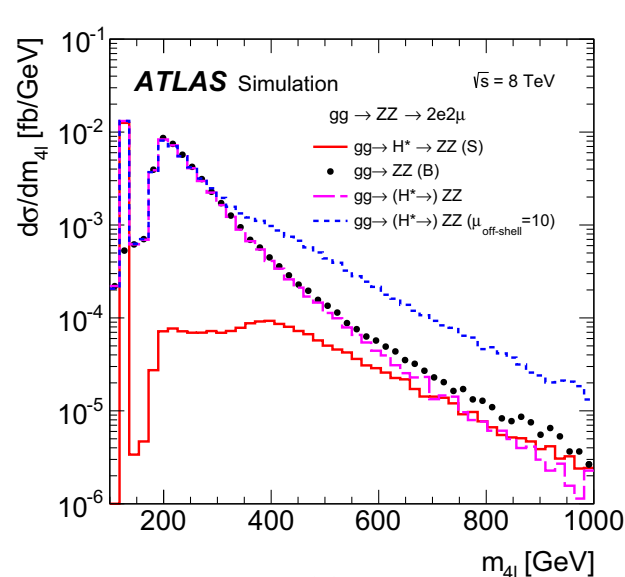

(a)

Fig. 2 a Differential cross-sections as a function of the fourlepton invariant mass $m_{4 \ell}$ in the range of $100 \mathrm{GeV}<m_{4 \ell}<$ $1000 \mathrm{GeV}$ for the $g g \rightarrow\left(H^{*} \rightarrow\right) Z Z \rightarrow 2 e 2 \mu$ channel at the parton level, for the $g g \rightarrow H^{*} \rightarrow Z Z$ signal (solid line), $g g \rightarrow Z Z$ continuum background (dots), gg $\rightarrow\left(H^{*} \rightarrow\right) Z Z$ with SM Higgs boson couplings (long-dashed line, including sig-

$125.36 \mathrm{GeV}$ [11], is assumed for the off-shell signal processes. This small difference has a negligible impact on the predicted off-shell production yields.

Figure 2 illustrates the size and kinematic properties of the gluon-induced signal and background processes by showing the four-lepton invariant mass $\left(m_{4 \ell}\right)$ distribution for the $g g \rightarrow\left(H^{*} \rightarrow\right) Z Z \rightarrow 2 e 2 \mu$ processes after applying the event selections in the $Z Z \rightarrow 4 \ell$ channel (see Sect. 3) on generator-level quantities. The process $g g \rightarrow\left(H^{*} \rightarrow\right) Z Z \rightarrow 2 e 2 \mu$ is shown for the SM $\mu_{\text {off-shell }}=1$ case and for an increased off-shell signal with $\mu_{\text {off-shell }}=10$. For low masses $m_{Z Z}<2 m_{Z}$ the offshell signal is negligible, while it becomes comparable to the continuum $g g \rightarrow Z Z$ background for masses above the $2 m_{t}$ threshold. The interference between the $g g \rightarrow$ $H^{*} \rightarrow Z Z$ signal and the $g g \rightarrow Z Z$ background is negative over the whole mass range. A very similar relation between the $g g \rightarrow H^{*} \rightarrow V V$ signal and the $g g \rightarrow$ $V V$ background is also seen for the $g g \rightarrow\left(H^{*} \rightarrow\right) Z Z$ $\rightarrow 2 \ell 2 v$ and $g g \rightarrow\left(H^{*} \rightarrow\right) W W \rightarrow e v \mu v$ processes.

The detector simulation for most generated Monte Carlo (MC) event samples is performed using Geant4 [26,27]. Some background MC samples in the $W W \rightarrow e v \mu \nu$ analysis for processes with large cross-sections are simulated with the fast detector simulation package Atlfast-II [27].

\subsection{Simulation of $g g \rightarrow\left(H^{*} \rightarrow\right) V V$}

To generate the $g g \rightarrow H^{*} \rightarrow V V$ and $g g \rightarrow V V$ processes, including the interference, the LO MC generators gg2VV $[7$, 28] and MCFM [9,10] together with PYTHIA8 [29] and SHERPA+OpenLoops [30-33] are used. The QCD renor-

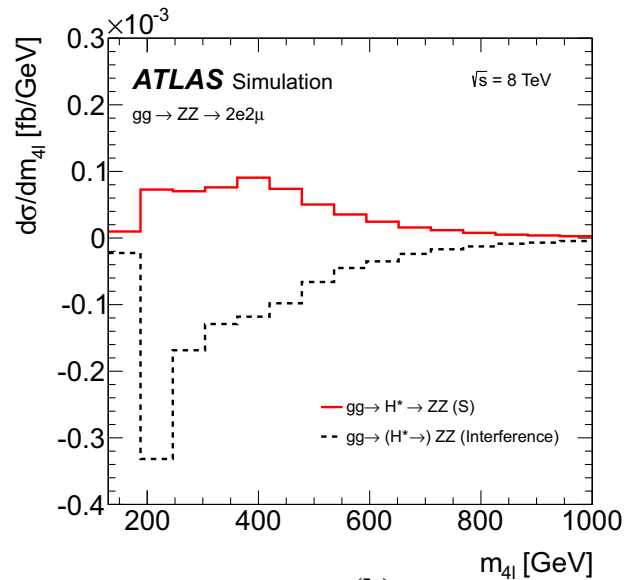

(b)

nal plus background plus interference) and $g g \rightarrow\left(H^{*} \rightarrow\right) Z Z$ with $\mu_{\text {off-shell }}=10$ (dashed line). $\mathbf{b}$ Differential cross-section as a function of $m_{4 \ell}$ in the range of $130 \mathrm{GeV}<m_{4 \ell}<1000 \mathrm{GeV}$ for the $\mathrm{SM} g g \rightarrow H^{*} \rightarrow Z Z \rightarrow 2 e 2 \mu$ signal (solid line) and its interference with the $g g \rightarrow Z Z \rightarrow 2 e 2 \mu$ continuum background (dashed line)

malisation and factorisation scales are set to $m_{V V} / 2$ [9]. The CT10 next-to-next-to-leading-order (NNLO) PDF set [34] is used, as the LO $g g \rightarrow V V$ process is part of the NNLO calculation for $p p \rightarrow V V$. The default parton showering and hadronisation option for the events processed with the full detector simulation is PYTHIA8 with the "power shower" parton shower option [29].

For the $g g \rightarrow H^{*} \rightarrow V V$ signal, a NNLO/LO K-factor ${ }^{2}$ including the next-to-leading-order (NLO) electroweak corrections, $\mathrm{K}^{H^{*}}\left(m_{V V}\right)=\sigma_{g g \rightarrow H^{*} \rightarrow V V}^{\mathrm{NNLO}} / \sigma_{g g \rightarrow H^{*} \rightarrow V V}^{\mathrm{LO}}$, is applied. The K-factor and associated uncertainties are calculated in Ref. [25] as a function of the Higgs boson virtuality $m_{V V}$ for $m_{H} \sim 125.5 \mathrm{GeV}$, using the MSTW2008 PDF set [35]. Additional corrections are used to re-weight the predictions to the CT10 NNLO PDF set used in the simulation.

For the $g g \rightarrow V V$ background and the interference with the $g g \rightarrow H^{*} \rightarrow V V$ signal, no higher-order QCD calculations are available. However, these corrections are studied for the $W W$ final state in Ref. [36] in the soft-collinear approximation, which is considered suitable for high-mass Higgs boson production. In this approximation, the signal K-factor is found to provide a reliable estimate for the higher-order QCD corrections to the signal-background interference term.

The K-factor for the $g g \rightarrow V V$ background process, $\mathrm{K}(g g \rightarrow V V)$, remains unknown. Therefore, the results in this note are given as a function of the unknown $\mathrm{K}$ factor ratio between the $g g \rightarrow V V$ background and the $g g \rightarrow H^{*} \rightarrow V V$ signal, defined as

\footnotetext{
${ }^{2}$ The shorter $g g \rightarrow X$ notation is used also in the context of higherorder QCD calculations where $q g$ and $q q$ initial states contribute to the full $p p \rightarrow X$ process.
} 
$\mathrm{R}_{H^{*}}^{B}=\frac{\mathrm{K}(g g \rightarrow V V)}{\mathrm{K}\left(g g \rightarrow H^{*} \rightarrow V V\right)}=\frac{\mathrm{K}^{\mathrm{B}}\left(m_{V V}\right)}{\mathrm{K}_{g g}^{H^{*}}\left(m_{V V}\right)}$,

where $\mathrm{K}^{\mathrm{B}}\left(m_{V V}\right)$ is the unknown mass-dependent $\mathrm{K}$-factor for the $g g \rightarrow V V$ background, and $\mathrm{K}_{g g}^{H^{*}}\left(m_{V V}\right)$ is the gluoninitiated $\mathrm{K}$-factor [25] for the signal ${ }^{3}$ as motivated by the soft-collinear approximation in Ref. [36]. Because the Kfactor $\mathrm{K}_{g g}^{H^{*}}\left(m_{V V}\right)$ changes by less than $10 \%$ as a function of $m_{V V}$ in the relevant region of phase space, no mass dependence on $\mathbf{R}_{H^{*}}^{B}$ is assumed. The range 0.5-2 is chosen for the variation of the $\mathrm{K}$-factor ratio $\mathrm{R}_{H^{*}}^{B}$ in order to include the full correction from the signal $\mathrm{K}$-factor $\mathrm{K}_{g g}^{H^{*}}\left(m_{V V}\right) \sim 2$ in the variation range. With respect to the $\mathrm{LO} g g \rightarrow V V$ process, this corresponds to an absolute variation in the approximate range 1-4. Using the $\mathrm{K}$-factors discussed above, the crosssection for the $g g \rightarrow\left(H^{*} \rightarrow\right) V V$ process with any off-shell Higgs boson signal strength $\mu_{\text {off-shell }}$ can be parameterised as:

$$
\begin{aligned}
& \sigma_{g g \rightarrow}\left(H^{*} \rightarrow\right) V V \\
&=\left.\mathrm{K}^{H^{*}}\left(m_{V V}\right) \cdot \mu_{\text {off-shell }}, m_{V V}\right) \\
&+\sqrt{\mathrm{K}_{g g}^{H^{*}}\left(m_{V V}\right) \cdot \mathrm{K}^{\mathrm{B}}\left(m_{V V}\right) \cdot \sigma_{g g \rightarrow H^{*} \rightarrow V V}}\left(m_{V V}\right) \\
&+\mathrm{K}^{\mathrm{B}}\left(m_{V V}\right) \cdot \sigma_{g g \rightarrow V} \rightarrow V, \text { cont } \\
&
\end{aligned}
$$

More details are given in Appendix A.1.

In addition, higher-order QCD corrections to the transverse momentum ${ }^{4} p_{\mathrm{T}}$ and the rapidity $y$ of the $V V$ system are studied using SHERPA+OpenLoops, which includes matrix-element calculations for the first hard jet emission. A difference of order $20 \%$ in the ratio of the $p_{\mathrm{T}}$ of the $V V$ system in the relevant kinematic region is observed when comparing the LO generators with parton shower to SHERPA+OpenLoops, while the difference in the rapidity $y$ of the $V V$ system is small. This difference in the $p_{\mathrm{T}}$ of the $V V$ system can modify the kinematic observables used in the analyses, leading to variations in both the kinematic shapes and acceptance which are not covered by the $m_{V V}$ dependent systematic uncertainties derived in Ref. [25]. To account for these effects, the LO generators are re-weighted

\footnotetext{
$\overline{{ }^{3} \text { Numerically, }} \mathrm{K}_{g g}^{H^{*}}\left(m_{V V}\right)$ differs from $\mathrm{K}^{H^{*}}\left(m_{V V}\right)$ by $\sim 2 \%$ as the higher-order QCD contribution from $q g$ and $q q$ production is small. However, $\mathrm{K}_{g g}^{H^{*}}\left(m_{Z Z}\right)$ has substantially larger uncertainties than $\mathrm{K}^{H^{*}}\left(m_{Z Z}\right)$. Therefore $\mathrm{K}^{H^{*}}\left(m_{Z Z}\right)$ is substituted here, ignoring the $2 \%$ shift in central value, but taking the difference in the systematic uncertainty into account.

4 ATLAS uses a right-handed coordinate system with its origin at the nominal interaction point (IP) in the centre of the detector, and the $z$-axis along the beam line. The $x$-axis points from the IP to the centre of the LHC ring, and the $y$-axis points upwards. Cylindrical coordinates $(r, \phi)$ are used in the transverse plane, $\phi$ being the azimuthal angle around the beam line. Observables labelled "transverse" are projected into the $x-y$ plane. The pseudorapidity is defined in terms of the polar angle $\theta$ as $\eta=-\ln \tan (\theta / 2)$.
}

to SHERPA+OpenLoops in the $p_{\mathrm{T}}$ of the $V V$ system. Due to the different jet emission mechanisms in the signal and the background processes, different re-weighting functions are derived for the $g g \rightarrow H^{*} \rightarrow V V$ signal, the $g g \rightarrow V V$ background, and the total $g g \rightarrow\left(H^{*}\right) \rightarrow V V$, respectively. The impact of the re-weighting on the acceptance is below $1 \%$ for the signal and at the level of 4-6 \% for the background. In the $Z Z \rightarrow 4 \ell$ channel, the re-weighting procedure is only used to account for the acceptance effects, as the matrix-element-based discriminant is insensitive to the $p_{\mathrm{T}}$ of the $Z Z$ system. For the $Z Z \rightarrow 2 \ell 2 v$ channel, the re-weighting is used in both the transverse mass shape and acceptance as the $m_{\mathrm{T}}$ depends on the $p_{\mathrm{T}}$ of the $Z Z$ system. For the $W W \rightarrow e v \mu \nu$ channel, the re-weighting affects only the acceptance.

\subsection{Simulation of electroweak $V V$ production through VBF and $V H$-like processes}

The electroweak ${ }^{5} p p \rightarrow V V+2 j$ processes contain both VBF-like events and $V H$-like events, which are simulated using MadGraph5 [37] and cross-checked using PHANTOM [38]. The QCD renormalisation and factorisation scales are set to $m_{W}$ following the recommendation in Ref. [39] and the CTEQ6L1 PDF set [40] is used. PYTHIA6 [41] is used for parton showering and hadronisation.

The high-mass range selected by this analysis includes Higgs boson signal events arising from:

- the off-shell VBF $H \rightarrow V V$ process, which scales with $\kappa_{V \text {,off-shell }}^{4}$ and is independent of $\Gamma_{H}$,

- VBF-like $V V$ processes with a $t$-channel Higgs boson exchange, which scale with $\kappa_{V \text {,off-shell }}^{4}$ and are independent of $\Gamma_{H}$,

- $W H$ and $Z H$ processes with an on-shell Higgs boson, with decays $Z \rightarrow 2 \ell$ or $W \rightarrow \ell v$ and $H \rightarrow 2 \ell 2 j$ or $H \rightarrow \ell v 2 j$, which scale with $\kappa_{V \text {,on-shell }}^{4} / \Gamma_{H}$,

where we assume the same coupling strength $\kappa_{V \text {,off-shell in }}$ the two VBF-like contributions, although the energy scale of the Higgs boson propagator is different between the two cases. Due to the different $\Gamma_{H}$ dependence, the on-shell and off-shell Higgs boson production processes are separated in the analysis by requiring that the generated Higgs boson mass satisfies $\left|m_{H}^{\text {gen. }}-125.5 \mathrm{GeV}\right|<1 \mathrm{GeV}$. This requirement is fully efficient in selecting the on-shell $V H$ process. The NNLO QCD corrected cross-section in Ref. [23] is used for the on-shell $V H$ production process. The cross-section $\sigma_{p p \rightarrow V V+2 j}\left(\mu_{\text {off-shell }}\right)$ for the electroweak $p p \rightarrow V V+2 j$ process for any off-shell Higgs boson signal strength $\mu_{\text {off-shell }}$

\footnotetext{
5 Electroweak means in this context that QCD diagrams that enter through the QCD NNLO corrections to $p p \rightarrow V V$ are not included.
} 
is parameterised in the same way as for the $g g \rightarrow\left(H^{*} \rightarrow\right)$ $V V$ process. Details are given in Appendix A.2.

\subsection{Simulation of $q \bar{q} \rightarrow Z Z, W W$ and $W Z$ backgrounds}

The $q \bar{q} \rightarrow Z Z, q \bar{q} \rightarrow W W$, and $q \bar{q} \rightarrow W Z$ backgrounds are simulated at NLO in QCD using POWHEG-BOX [42] with dynamic QCD renormalisation and factorisation scales of $m_{V V^{\left({ }^{\prime}\right)}}$ and the CT10 NLO PDF set. In addition, SHERPA is used as a cross-check for the $q \bar{q} \rightarrow W Z$ background. Parton showering and hadronisation are done with PYTHIA8 for $q \bar{q} \rightarrow V Z$ and PYTHIA6 for $q \bar{q} \rightarrow W W$. The interference between the $q \bar{q} \rightarrow W W$ and $q \bar{q} \rightarrow Z Z$ processes for the $2 \ell 2 v$ final state is negligible [42] and thus not considered.

The cross-sections for the $q \bar{q} \rightarrow Z Z$ and $q \bar{q} \rightarrow W W$ processes are calculated in Refs. [43,44], respectively, for two on-shell $Z$ or $W$ bosons in the final state at NNLO QCD accuracy. As these calculations include the $g g \rightarrow V V$ processes as part of the NNLO calculation, a different K-factor is provided by the authors of the Refs. [43,44] excluding the $g g \rightarrow V V$ component and using a QCD renormalisation and factorisation scale $\mu_{\mathrm{QCD}}$ of $m_{V V} / 2$ in order to consistently match the simulation of the $g g \rightarrow\left(H^{*} \rightarrow\right) V V$ process:

$$
\begin{aligned}
\mathrm{K}_{q \bar{q}}\left(m_{V V}\right)= & {\left[\sigma_{q \bar{q} \rightarrow V V}^{\mathrm{NNLO}}\left(m_{V V}, \mu_{\mathrm{QCD}}=m_{V V} / 2\right)\right.} \\
& \left.-\sigma_{g g \rightarrow V V}^{\mathrm{LO}}\left(m_{V V}, \mu_{\mathrm{QCD}}=m_{V V} / 2\right)\right] \\
& / \sigma_{q \bar{q} \rightarrow V V}^{\mathrm{NLO}}\left(m_{V V}, \mu_{\mathrm{QCD}}=m_{V V}\right)
\end{aligned}
$$

Electroweak higher-order corrections are not included in POWHEG-BOX. These corrections are calculated in Refs. $[45,46]$ for on-shell outgoing vector bosons and found to be about $-10 \%$ in the high-mass $V V$ region of this analysis. To account for these corrections, the POWHEG-BOX events are re-weighted using a procedure comparable to the one described in Ref. [47], based on the kinematics of the diboson system and the initial state quarks.

\subsection{Simulation of top-quark backgrounds}

In the $W W \rightarrow e v \mu \nu$ channel, the $t \bar{t}$ and single-top ( $s$ channel and $W t$ ) backgrounds are simulated with POWHEGBOX $[48,49]$ with parton showering and hadronisation done with PYTHIA6, using the CT10 NLO PDF set. The $t$ channel single-top background is simulated using AcerMC [50]+PYTHIA6 and uses the CTEQ6LI PDF set. The relative rates of $t \bar{t}$ and single-top production are evaluated with Top ++2.0 [51] and the calculations in Refs. [52-54] respectively.

\section{Analysis of the $Z Z \rightarrow 4 \ell$ final state}

The analysis for the $Z Z \rightarrow 4 \ell$ final state closely follows the Higgs boson measurements in the same final state described in Ref. [55], with the same physics object definitions, trigger and event selections, and background estimation methods. A matrix-element-based discriminant (ME-based discriminant) is constructed to enhance the separation between the $g g \rightarrow H^{*} \rightarrow Z Z$ signal and the $g g \rightarrow Z Z$ and $q \bar{q} \rightarrow Z Z$ backgrounds, and is subsequently used in a binned maximum-likelihood fit for the final result.

\subsection{Event selection}

To minimise the dependence of the $g g \rightarrow Z Z$ kinematics on higher-order QCD effects, the analysis is performed inclusively, ignoring the number of jets in the events.

The analysis is split into four lepton channels $(2 \mu 2 e$, $2 e 2 \mu, 4 e, 4 \mu$ ) as in Ref. [55]. Each electron (muon) must satisfy $E_{\mathrm{T}}>7 \mathrm{GeV}\left(p_{\mathrm{T}}>6 \mathrm{GeV}\right)$ and be measured in the pseudorapidity range $|\eta|<2.47(|\eta|<2.7)$. The highest- $p_{\mathrm{T}}$ lepton in the quadruplet must satisfy $p_{\mathrm{T}}>20 \mathrm{GeV}$, and the second (third) lepton in $p_{\mathrm{T}}$ order must satisfy $p_{\mathrm{T}}>15 \mathrm{GeV}$ $\left(p_{\mathrm{T}}>10 \mathrm{GeV}\right)$. Lepton pairs are formed from same-flavour opposite-charge leptons. For each channel, the lepton pair with the mass closest to the $Z$ boson mass is referred to as the leading dilepton pair and its invariant mass, $m_{12}$, is required to be between 50 and $106 \mathrm{GeV}$. The second (subleading) pair is chosen from the remaining leptons (more than four leptons are allowed per event) as the pair closest in mass to the $Z$ boson and in the range of $50 \mathrm{GeV}<m_{34}<115 \mathrm{GeV}$. The off-peak region is defined to include the range from $220 \mathrm{GeV}<m_{4 \ell}<1000 \mathrm{GeV}$.

Figure 3a shows the observed and expected distributions of $m_{4 \ell}$ combining all lepton channels in the full off-peak region. The data are in agreement with the SM predictions, with a small deficit of the order of one standard deviation $(1 \sigma)$. Table 1 shows the expected and observed number of events in the signal-enriched region, $400 \mathrm{GeV}<m_{4 \ell}<$ $1000 \mathrm{GeV}$, combining all lepton channels. This mass region was chosen since it is optimal for a $m_{4 \ell}$ cut-based analysis.

\subsection{Matrix-element-based kinematic discriminant}

The matrix-element kinematic discriminant fully exploits the event kinematics in the centre-of-mass frame of the $4 \ell$ system, based on eight observables: $\left\{m_{4 \ell}, m_{12}, m_{34}, \cos \theta_{1}\right.$, $\left.\cos \theta_{2}, \phi, \cos \theta^{*}, \phi_{1}\right\}$, defined in Refs. [5,55]. These observables are used to create the four-momenta of the leptons and incoming partons, which are then used to calculate matrix elements for different processes, provided by the MCFM pro- 


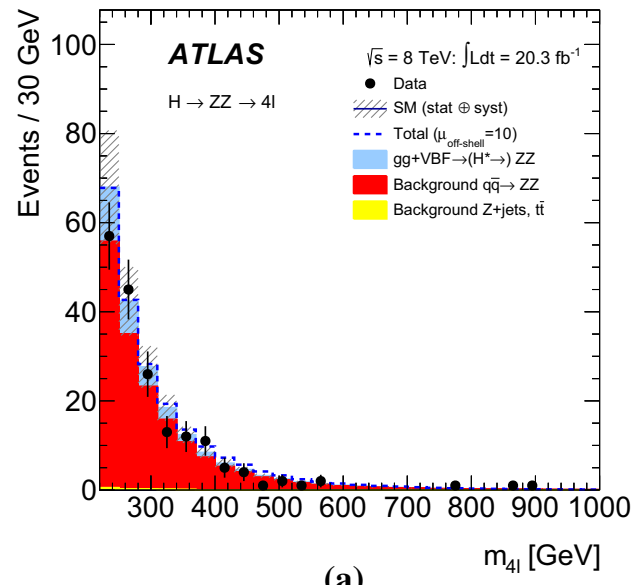

(a)

Fig. 3 Observed distributions for a the four-lepton invariant mass $m_{4 \ell}$ in the range of $220 \mathrm{GeV}<m_{4 \ell}<1000 \mathrm{GeV}$ and b the ME-based discriminant combining all lepton final states for the ME-based analysis signal region, compared to the expected contributions from the SM including the Higgs boson (stack). The dashed line corresponds to the

Table 1 Expected and observed numbers of events in the signal region for all final states in the cut-based approaches. For the $Z Z \rightarrow 4 \ell$ analysis a mass range of $400<m_{4 \ell}<1000 \mathrm{GeV}$ is used. The other backgrounds in the $Z Z \rightarrow 4 \ell$ final state include contributions from $Z+$ jets and top-quark processes. For the $Z Z \rightarrow 2 \ell 2 v$ analysis the range $380 \mathrm{GeV}<m_{\mathrm{T}}^{Z Z}<1000 \mathrm{GeV}$ is considered. For the $W W \rightarrow e v \mu \nu$ analysis, the region $\mathrm{R}_{8}>450 \mathrm{GeV}$ is used and background event yields are quoted after the likelihood fit was performed. The expected events

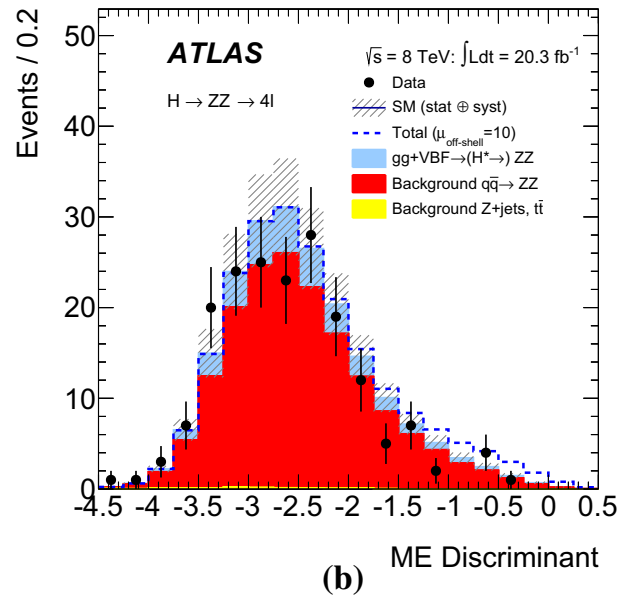

total expected event yield, including all backgrounds and the Higgs boson with $\mu_{\text {off-shell }}=10$. A relative $g g \rightarrow Z Z$ background $\mathrm{K}$-factor of $\mathrm{R}_{H^{*}}^{B}=1.0$ is assumed. The $Z+$ jets and top-quark backgrounds are barely visible in the plot since they are very small $(<1 \%$ of the total background)

for the $g g \rightarrow\left(H^{*} \rightarrow\right) V V$ and $\operatorname{VBF}\left(H^{*} \rightarrow\right) V V$ processes $(Z Z$ or $W W$ ), including the Higgs boson signal, background and interference, are reported for both the SM predictions (in bold) and $\mu_{\text {off-shell }}=10$. A relative $g g \rightarrow V V$ background $\mathrm{K}$-factor of $\mathrm{R}_{H^{*}}^{B}=1$ is assumed. The uncertainties in the number of expected events include the statistical uncertainties from MC samples and systematic uncertainties. The entries with $\mathrm{a}-$ are for processes with event yields $<0.1$

\begin{tabular}{|c|c|c|c|}
\hline Process & $Z Z \rightarrow 4 \ell$ & $Z Z \rightarrow 2 \ell 2 v$ & $W W \rightarrow e v \mu \nu$ \\
\hline$g g \rightarrow H^{*} \rightarrow V V(\mathrm{~S})$ & $1.1 \pm 0.3$ & $3.2 \pm 1.0$ & $1.5 \pm 0.4$ \\
\hline$g g \rightarrow V V(\mathrm{~B})$ & $2.8 \pm 0.8$ & $5.3 \pm 1.6$ & $3.6 \pm 1.1$ \\
\hline$g g \rightarrow\left(H^{*} \rightarrow\right) V V$ & $2.4 \pm 0.7$ & $3.9 \pm 1.2$ & $2.4 \pm 1.2$ \\
\hline$g g \rightarrow\left(H^{*} \rightarrow\right) V V\left(\mu_{\text {off-shell }}=10\right)$ & $9.2 \pm 2.5$ & $24.0 \pm 7.3$ & $10 \pm 4$ \\
\hline $\mathrm{VBF} H^{*} \rightarrow V V(\mathrm{~S})$ & $0.12 \pm 0.01$ & $0.48 \pm 0.04$ & $0.42 \pm 0.05$ \\
\hline VBF $V V(\mathrm{~B})$ & $0.71 \pm 0.04$ & $1.2 \pm 0.2$ & $1.6 \pm 0.2$ \\
\hline $\operatorname{VBF}\left(H^{*} \rightarrow\right) \boldsymbol{V} V$ & $0.59 \pm \mathbf{0 . 0 3}$ & $0.7 \pm 0.1$ & $1.1 \pm 0.1$ \\
\hline $\operatorname{VBF}\left(H^{*} \rightarrow\right) V V\left(\mu_{\text {off-shell }}=10\right)$ & $1.17 \pm 0.06$ & $2.9 \pm 0.2$ & $2.8 \pm 0.3$ \\
\hline$q \bar{q} \rightarrow Z Z$ & $21.3 \pm 2.1$ & $31.5 \pm 3.5$ & \multirow{2}{*}{$2.0 \pm 0.2$} \\
\hline$q \bar{q} \rightarrow W Z$ & - & $10.6 \pm 1.4$ & \\
\hline$q \bar{q} \rightarrow W W$ & - & ) & $40 \pm 5$ \\
\hline$t \bar{t}, W t$, and $t \bar{b} / t q \bar{b}$ & - & $0.4 \pm 0.2$ & $35 \pm 4$ \\
\hline$Z \rightarrow \tau \tau$ & - & $\int$ & $1.4 \pm 0.2$ \\
\hline$Z \rightarrow e e, \mu \mu$ & - & $3.5 \pm 3.0$ & - \\
\hline Other backgrounds & - & $0.8 \pm 0.2$ & $8.7 \pm 1.3$ \\
\hline Total Expected (SM) & $24.4 \pm 2.2$ & $51 \pm 6$ & $90 \pm 4$ \\
\hline Observed & 18 & 48 & 82 \\
\hline
\end{tabular}

gram [9]. The following matrix elements are calculated for each event in the mass range $220 \mathrm{GeV}<m_{4 \ell}<1000 \mathrm{GeV}$ :

- $P_{q \bar{q}}$ : matrix element squared for the $q \bar{q} \rightarrow Z Z \rightarrow 4 \ell$ process,
- $P_{g g}$ : matrix element squared for the $g g \rightarrow\left(H^{*} \rightarrow\right) Z Z$ $\rightarrow 4 \ell$ process including the Higgs boson $\left(m_{H}=\right.$ 125.5 GeV) with SM couplings, continuum background and their interference, 
- $P_{H}$ : matrix element squared for the $g g \rightarrow H^{*} \rightarrow Z Z \rightarrow$ $4 \ell$ process $\left(m_{H}=125.5 \mathrm{GeV}\right)$.

The kinematic discriminant is defined as in Ref. [9]:

$\mathrm{ME}=\log _{10}\left(\frac{P_{H}}{P_{g g}+c \cdot P_{q \bar{q}}}\right)$,

where $c=0.1$ is an empirical constant, to approximately balance the overall cross-sections of the $q \bar{q} \rightarrow Z Z$ and $g g \rightarrow\left(H^{*} \rightarrow\right) Z Z$ processes. The value of $c$ has a very small effect on the analysis sensitivity. Figure $3 \mathrm{~b}$ shows the observed and expected distributions of the ME-based discriminant combining all lepton final states. Events with the ME-based discriminant value between -4.5 and 0.5 are selected with a signal efficiency of $>99 \%$.

In addition, an alternative multivariate discriminant based on a boosted decision tree (BDT) algorithm was studied to further separate the $g g \rightarrow H^{*} \rightarrow Z Z$ signal and the main $q \bar{q} \rightarrow Z Z$ background, by exploiting additional kinematic information ( $p_{\mathrm{T}}$ and $\eta$ ) of the $Z Z$ system. The analysis sensitivity improves very little ( $\sim 2 \%$ ) compared to the ME-based discriminant alone. Due to the dependence on the $p_{\mathrm{T}}$ of the $Z Z$ system, the BDT-based discriminant introduces additional systematic uncertainties from the higher-order QCD corrections. For these reasons, the BDT-based discriminant is not used for the final result.

\section{Analysis of the $Z Z \rightarrow 2 \ell 2 v$ final state}

The analysis of the $Z Z \rightarrow 2 \ell 2 v$ channel follows strategies similar to those used in the invisible Higgs boson search in the $Z H$ channel [20]. The definitions of the reconstructed physics objects (electrons, muons, jets, and missing transverse momentum) are identical, but some of the kinematic cuts were optimised for the current analysis.

\subsection{Event selection}

As the neutrinos in the final state do not allow for a kinematic reconstruction of $m_{Z Z}$, the transverse mass $\left(m_{\mathrm{T}}^{Z Z}\right)$ reconstructed from the transverse momentum of the dilepton system $\left(p_{\mathrm{T}}^{\ell \ell}\right)$ and the magnitude of the missing transverse momentum $\left(E_{\mathrm{T}}^{\text {miss }}\right)$ :

$$
m_{\mathrm{T}}^{Z Z} \equiv \sqrt{\left(\sqrt{m_{Z}^{2}+\left|\boldsymbol{p}_{\mathrm{T}}^{\ell \ell}\right|^{2}}+\sqrt{m_{Z}^{2}+\left|\boldsymbol{E}_{\mathrm{T}}^{\mathrm{miss}}\right|^{2}}\right)^{2}-\left|\boldsymbol{p}_{\mathrm{T}}^{\ell \ell}+\boldsymbol{E}_{\mathrm{T}}^{\mathrm{miss}}\right|^{2}}
$$

is chosen as the discriminating variable to enhance sensitivity to the $g g \rightarrow H^{*} \rightarrow Z Z$ signal.

The selection criteria are optimised to maximise the signal significance with respect to the main backgrounds, which are $Z Z, W Z, W W$, top-quark, and $W / Z+$ jets events, as described in Sect. 4.2. The impact of the background uncertainty is considered in the significance calculation.

First, events with two oppositely charged electron or muon candidates in the $Z$ mass window $76 \mathrm{GeV}<m_{\ell \ell}<106 \mathrm{GeV}$ are selected. Events with a third lepton $(e$ or $\mu)$ identified using looser identification criteria for the electrons and a lower $p_{\mathrm{T}}$ threshold of $7 \mathrm{GeV}$ are rejected. A series of selection requirements are necessary to suppress the DrellYan background, including: $E_{\mathrm{T}}^{\text {miss }}>180 \mathrm{GeV} ; 380 \mathrm{GeV}<$ $m_{\mathrm{T}}^{Z Z}<1000 \mathrm{GeV}$; the azimuthal angle between the transverse momentum of the dilepton system and the missing transverse momentum $\Delta \phi\left(p_{\mathrm{T}}^{\ell \ell}, E_{\mathrm{T}}^{\text {miss }}\right)>2.5$; and || $\boldsymbol{E}_{\mathrm{T}}^{\text {miss }}+$ $\sum_{\text {jet }} \boldsymbol{p}_{\mathrm{T}}^{\text {jet }}\left|-p_{\mathrm{T}}^{\ell \ell}\right| / p_{\mathrm{T}}^{\ell \ell}<0.3$ Events with a $b$-jet with $p_{\mathrm{T}}$ $>20 \mathrm{GeV}$ and $|\eta|<2.5$, identified by the MV1 algorithm [56,57] with $70 \%$ tagging efficiency, are rejected to suppress the top-quark background. Finally, the selection on the azimuthal angle between the two leptons $\Delta \phi_{\ell \ell}<1.4$ is applied to select events with boosted $Z$ bosons to further discriminate the signal from the background.

\subsection{Background estimation}

The dominant background is $q \bar{q} \rightarrow Z Z$ production, followed by $q \bar{q} \rightarrow W Z$ production. Background contributions from events with a genuine isolated lepton pair, not originating from a $Z \rightarrow e e$ or $Z \rightarrow \mu \mu$ decay, arise from the $W W, t \bar{t}$, $W t$, and $Z \rightarrow \tau \tau$ processes. The remaining backgrounds are from $Z \rightarrow e e$ or $Z \rightarrow \mu \mu$ decays with poorly reconstructed $E_{\mathrm{T}}^{\mathrm{miss}}$, and from events with at least one misidentified electron or muon coming from $W+$ jets, semileptonic top decays ( $t \bar{t}$ and single top), and multi-jet events.

The $q \bar{q} \rightarrow Z Z$ background is estimated in the same way as for the $Z Z \rightarrow 4 \ell$ analysis using the POWHEG-BOX simulation as described in Sect. 2.3. The $W Z$ background is also estimated with the simulation (described in Sect. 2.3) and validated with data in a three-lepton control region. The observed number of events in the control region for $E_{\mathrm{T}}^{\mathrm{miss}}>$ $180 \mathrm{GeV}$ (300 GeV) is 30 (3), whereas the predicted event yield is $22.9 \pm 0.8(3.4 \pm 0.3)$. No significant difference is observed between the data and simulation.

The $W W, t \bar{t}, W t$, and $Z \rightarrow \tau \tau$ backgrounds are inclusively estimated with data assuming lepton flavour symmetry in an $e \mu$ control region using a relaxed selection. The following equations show how these backgrounds in the signal region can be estimated with $e \mu$ events:

$N_{e e}^{\mathrm{bkg}}=\frac{1}{2} \times N_{e \mu}^{\text {data, sub }} \times \alpha$,
$N_{\mu \mu}^{\mathrm{bkg}}=\frac{1}{2} \times N_{e \mu}^{\text {data, sub }} \times \frac{1}{\alpha}$,

where $N_{e e}^{\mathrm{bkg}}$ and $N_{\mu \mu}^{\mathrm{bkg}}$ are the number of dielectron and dimuon events in the signal region. $N_{e \mu}^{\text {data,sub }}$ is the num- 
ber of events in the $e \mu$ control region with $W Z, Z Z$, and other small backgrounds ( $W+$ jets, $t \bar{t} V$, and triboson) subtracted using simulation. The different $e$ and $\mu$ efficiencies are taken into account using the $\alpha$ variable, which is an efficiency correction factor determined from the ratio of dielectron to dimuon event yields after the inclusive $Z$ mass requirement $\left(76 \mathrm{GeV}<m_{\ell \ell}<106 \mathrm{GeV}\right)$. The measured value of $\alpha$ is 0.942 with a systematic uncertainty of 0.004 and a negligible statistical uncertainty. This scale factor is applied to the MC predictions. The other source of systematic uncertainty comes from the subtraction of $W Z, Z Z$, and other small backgrounds in the $e \mu$ control region using the simulation. As no data event remains after applying the full selection, a scale factor of $1.4 \pm 0.3$ is derived by comparing the event yields from the data-driven and MC predictions with a relaxed selection applying the $E_{\mathrm{T}}^{\mathrm{miss}}$ and $m_{\mathrm{T}}^{Z Z}$ requirements but no further cuts. Experimental systematic uncertainties are considered for the MC predictions.

Imperfect modelling of detector non-uniformities and $E_{\mathrm{T}}^{\text {miss }}$ response could lead to an incorrect estimate of the $Z$ boson background in the signal region. The $Z$ boson background is estimated with data using the two-dimensional sideband regions constructed by reversing one or both of the $\Delta \phi\left(p_{\mathrm{T}}^{\ell \ell}, E_{\mathrm{T}}^{\mathrm{miss}}\right)$ and $\Delta \phi_{\ell \ell}$ selections [20]. The main uncertainty on the mis-measured $Z$ boson background arises from the differences in shape of the $E_{\mathrm{T}}^{\mathrm{miss}}$ and $m_{\mathrm{T}}^{Z Z}$ distributions in the signal and sideband regions and the small correlation between these two variables. Other systematic uncertainties originate from the subtraction of the non- $Z$ boson backgrounds in the sideband regions.

The $W+$ jets and multi-jet backgrounds are estimated from data using the fake-factor method [20]. The predicted background with a looser $E_{\mathrm{T}}^{\mathrm{miss}}$ selection applied at $100 \mathrm{GeV}$, and without the $m_{\mathrm{T}}^{Z Z}$ selection, is $0.04 \pm 0.01$ events. No event remains after applying the full event selection for both the data-driven method and MC samples, and hence this background is estimated to be negligible.

The predicted signals and backgrounds with statistical and systematic uncertainties are summarised in Table 1. The observed event yields agree with the total predicted ones from the SM within the uncertainties. Figure 4 shows the distributions of $m_{\mathrm{T}}^{Z Z}$ for the $e e$ and $\mu \mu$ channels in the signal region, compared to the predicted contributions from the SM as well as to a Higgs boson with $\mu_{\text {off-shell }}=10$.

\section{Analysis of the $W W \rightarrow e v \mu v$ final state}

The analysis of the $W W \rightarrow e v \mu \nu$ channel closely follows the Higgs boson measurements in the oppositely charged electron-muon pair final state in Ref. [58]. This selection ensures orthogonality with the $Z Z \rightarrow 2 \ell 2 v$ final state. The same object identification and selection as in Ref. [58] is used

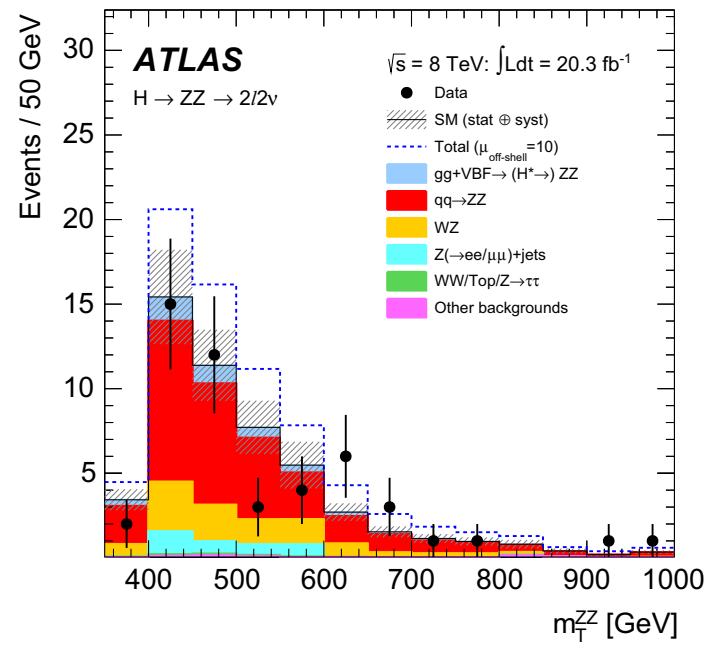

Fig. 4 Observed distribution of the $Z Z$ transverse mass $m_{\mathrm{T}}^{Z Z}$ in the range $380 \mathrm{GeV}<m_{\mathrm{T}}^{Z Z}<1000 \mathrm{GeV}$ combining the $2 e 2 v$ and $2 \mu 2 v$ channels, compared to the expected contributions from the SM including the Higgs boson (stack). The first bin only contains events in the range $380 \mathrm{GeV}<m_{\mathrm{T}}^{Z Z}<400 \mathrm{GeV}$. The hatched area shows the combined statistical and systematic uncertainties. The dashed line corresponds to the total expected event yield, including all backgrounds and the Higgs boson with $\mu_{\text {off-shell }}=10$. A relative $g g \rightarrow Z Z$ background $\mathrm{K}$-factor of $\mathrm{R}_{H^{*}}^{B}=1$ is assumed

in this analysis. Additionally, an event selection identical to that used for the gluon fusion initial states in $H \rightarrow W W \rightarrow$ $e v \mu \nu$ is used, up to and including a requirement on missing transverse momentum: leading lepton $p_{\mathrm{T}}>22 \mathrm{GeV}$, subleading lepton $p_{\mathrm{T}}>10 \mathrm{GeV}, m_{\ell \ell}>10 \mathrm{GeV}$, and $p_{\mathrm{T}}^{\text {miss,track }}>20 \mathrm{GeV}$, the magnitude of the missing transverse momentum, with a track-based soft term. The signal region (SR) and background estimations were revised for the high-mass region used in this analysis. Contrary to the base analysis [58], events are not binned by the number of jets. Top-quark events and SM $W W$ production remain the largest expected backgrounds.

\subsection{Event selection}

As with the $Z Z \rightarrow 2 \ell 2 v$ channel, the neutrinos in the final state do not allow for a kinematic reconstruction of $m_{V V}$. Thus a transverse mass $\left(m_{\mathrm{T}}^{W W}\right)$ is calculated from the dilepton system transverse energy $\left(E_{\mathrm{T}}^{\ell \ell}\right)$, the vector sum of lepton transverse momenta $\left(\boldsymbol{p}_{\mathrm{T}}^{\ell \ell}\right)$, and the vector sum of neutrino transverse momenta $\left(\boldsymbol{p}_{\mathrm{T}}^{\nu v}\right)$, measured with $p_{\mathrm{T}}^{\text {miss, track }}$ :

$$
\begin{aligned}
& m_{\mathrm{T}}^{W W}= \sqrt{\left(E_{\mathrm{T}}^{\ell \ell}+p_{\mathrm{T}}^{\nu v}\right)^{2}-\left|\boldsymbol{p}_{\mathrm{T}}^{\ell \ell}+\boldsymbol{p}_{\mathrm{T}}^{\nu v}\right|^{2}}, \\
& \text { where } E_{\mathrm{T}}^{\ell \ell}=\sqrt{\left(p_{\mathrm{T}}^{\ell \ell}\right)^{2}+\left(m_{\ell \ell}\right)^{2}} .
\end{aligned}
$$

The transverse mass is modified compared to the definition in Eq. (8) as the neutrinos do not come from the same parent particle, and there is no $m_{Z}$ constraint. 

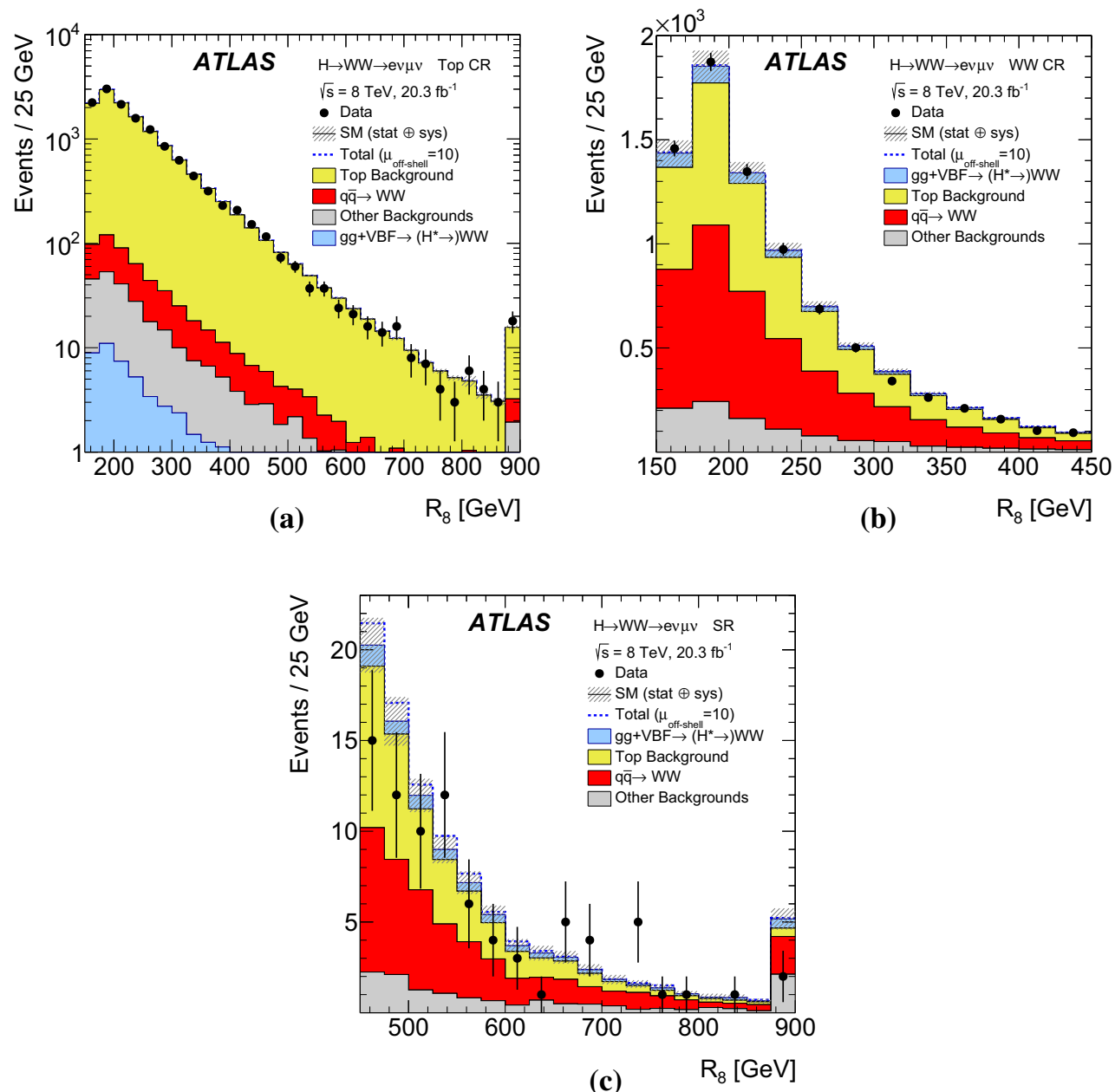

Fig. 5 Observed distributions of $\mathrm{R}_{8}$, constructed from the dilepton invariant mass and transverse mass, Eq. (11), in the $W W \rightarrow e v \mu \nu$ channel for a the top control region, $\mathbf{b} W W$ control region (the CRs start at $160 \mathrm{GeV}$ ), and $\mathbf{c}$ the signal region for $\mathrm{R}_{8}$ above $450 \mathrm{GeV}$, compared to the expected contributions from the SM including the Higgs boson (stack). The dashed line corresponds to the total expected event yield,

In order to isolate the off-shell Higgs boson production while minimising the impact of higher-order QCD effects on $g g \rightarrow W W$ kinematics, a new variable, $\mathrm{R}_{8}$, is introduced:

$\mathrm{R}_{8}=\sqrt{m_{\ell \ell}^{2}+\left(a \cdot m_{\mathrm{T}}^{W W}\right)^{2}}$.

Both the coefficient $a=0.8$ and the requirement $\mathrm{R}_{8}>$ $450 \mathrm{GeV}$ are optimised for off-shell signal sensitivity while also rejecting on-shell Higgs boson events, which have relatively low values of $m_{\ell \ell}$ and $m_{\mathrm{T}}^{W W}$. The predicted on-shell signal contamination is $0.04 \pm 0.03$ (stat.) events. The MV1 algorithm, at $85 \%$ efficiency, is used to reject $b$-jets with $p_{\mathrm{T}}>20 \mathrm{GeV}$ and $|\eta|<2.4$ in order to reject backgrounds containing top quarks. A more efficient working point for $b$-jet tagging is used compared to the $Z Z \rightarrow 2 \ell 2 v$ analysis because of the need to reject a substantially larger top- including all backgrounds and the Higgs boson with $\mu_{\text {off-shell }}=10$. The last bin in a and $\mathbf{c}$ includes the overflow. A relative $g g \rightarrow W W$ background $\mathrm{K}$-factor of $\mathrm{R}_{H^{*}}^{B}=1$ is assumed. The top-quark and $W W$ backgrounds are normalised to data as described in Sect. 5.1. The stacking order follows the legend in each plot

quark background. A requirement on the separation between leptons, $\Delta \eta_{\ell \ell}<1.2$, suppresses $q \bar{q}$-initiated $W W$ production relative to $g g$-initiated production. The $b$-jet veto and $\Delta \eta_{\ell \ell}$ requirement are found to have a minimal impact on the $W W$-system kinematics and jet multiplicity in the $g g \rightarrow\left(H^{*} \rightarrow\right) W W$ processes. Table 1 contains the predicted and observed event yields in the signal region, $90 \pm 4$ and 82 respectively, in agreement with the SM with a small deficit in data. The distribution of the $\mathrm{R}_{8}$ variable in the signal region is shown in Fig. 5c for the SM expectation and for a Higgs boson with $\mu_{\text {off-shell }}=10$.

\subsection{Background estimation}

The dominant backgrounds arise from processes with real $W$ bosons in the final state. The two backgrounds with the largest expected event yield are top-quark and $q \bar{q} \rightarrow W W$ 
production. Dedicated control regions (CRs) are constructed to normalise these two backgrounds in the signal region with a simultaneous fit. Uncertainties on the extrapolation from the CRs to the signal region are described in Sects. 6.2 and 6.3 .

The top-quark background predictions in the signal and $W W$ control region are both normalised from the same top CR. A sample of top-quark events is obtained by starting from the signal region and reversing the $b$-jet veto by requiring exactly one $b$-tagged jet. This is closer in phase space to the $b$-jet-vetoed signal region than requiring at least one $b$-tag and results in a smaller uncertainty. The statistical error on the top-quark background normalisation is reduced by expanding the top CR down to $\mathrm{R}_{8}>160 \mathrm{GeV}$ and dropping the $\Delta \eta_{\ell \ell}$ requirement. The impact of these changes is discussed in Sect. 6.3. An event yield of 13498 events is observed in the top CR (Fig. 5a), resulting in a fit normalisation factor of $1.03 \pm$ 0.04 , where the uncertainty includes all systematic sources, including extrapolation uncertainties described in Sect. 6.3. The top CR is approximately $96 \%$ pure in top-quark events.

The $q \bar{q} \rightarrow W W$ background is normalised to data using an additional $\mathrm{CR}$. The region $160 \mathrm{GeV}<\mathrm{R}_{8}<450 \mathrm{GeV}$ without the $\Delta \eta_{\ell \ell}$ requirement is used because it has a large $W W$ contribution with negligible on-shell Higgs boson contamination and is adjacent to the signal region. A $b$-jet veto is applied to reject part of the substantial top-quark contamination. An event yield of 8007 events is observed in the $W W \mathrm{CR}$ (Fig. 5b), resulting in a fit normalisation factor of $1.03 \pm 0.11$, including all of the uncertainties as above. This $\mathrm{CR}$ is approximately $46 \%$ pure in $q \bar{q} \rightarrow W W$, while the leading background of top-quark events contributes $39 \%$. The $g g$-initiated $W W$ background is estimated from MC simulation, as discussed in Sect. 2.1.

The remaining background predictions, except for $W+$ jets and multi-jet production, are taken from MC simulation, as described in Ref. [58]. The predicted fraction of the total background in the signal region arising from $g g \rightarrow W W$, $W+$ jets, and $W \gamma / W \gamma^{*} / W Z / Z Z$ events is approximately $4 \%$ each, while for $Z+$ jets it is $2 \%$. The $W+$ jets and multijet backgrounds are estimated by applying a data-driven extrapolation factor to CRs with lepton candidates failing the nominal lepton identification and isolation, while passing a loosened requirement [58].

\section{Systematic uncertainties}

The largest systematic uncertainties for this analysis arise from theoretical uncertainties on the $g g \rightarrow H^{*} \rightarrow V V$ signal process, the $g g / q \bar{q} \rightarrow V V$ background processes and the interference between the $g g \rightarrow V V$ signal and background processes. The electroweak $\left(H^{*} \rightarrow V V\right)$ processes in association with two jets contribute about $10-30 \%$ of the total signal. The associated theoretical uncertainties due to the missing higher-order corrections and PDF variations are small for $V H$-like and VBF-like processes $p p \rightarrow Z Z+2 j$, and are therefore not included in the analysis. Compared to the theoretical uncertainties, the experimental uncertainties are small in the $Z Z \rightarrow 2 \ell 2 \nu$ and $W W \rightarrow e v \mu \nu$ analyses and close to negligible in the $Z Z \rightarrow 4 \ell$ analysis. In the $Z Z \rightarrow 2 \ell 2 \nu$ and $W W \rightarrow e v \mu \nu$ analyses, uncertainties on the extrapolations from the control regions to the signal regions are included.

\subsection{Systematic uncertainties on $g g \rightarrow\left(H^{*} \rightarrow\right) V V$}

The uncertainty from missing higher-order QCD and EW corrections to the off-shell $g g \rightarrow H^{*} \rightarrow V V$ signal is estimated in Ref. [25] as a function of the Higgs boson virtuality, $m_{V V}$, and adopted for this analysis. The uncertainty is 20$30 \%$ for the high-mass region used in this analysis. The PDF uncertainty for the $g g \rightarrow\left(H^{*} \rightarrow\right) V V$ process as a function of $m_{V V}$ is found to be 10-20\% in the high-mass region used in this analysis. This is consistent with an earlier study at $\sqrt{s}=7 \mathrm{TeV}$ [39].

For the $g g \rightarrow V V$ background, higher-order QCD calculations are not available. As discussed in Sect. 2.1, the gluoninduced part of the signal $\mathrm{K}$-factor $\mathrm{K}_{g g}^{H^{*}}\left(m_{V V}\right)$ is applied to the background and results are then given as a function of the unknown $\mathrm{K}$-factor ratio $\mathrm{R}_{H^{*}}^{B}$ between background and signal. The uncertainty on $\mathrm{K}_{g g}^{H^{*}}\left(m_{V V}\right)$ is larger than the uncertainty on $\mathrm{K}^{H^{*}}\left(m_{V V}\right)$ because some contributions to the full signal NNLO QCD K-factor are not present in $\mathrm{K}_{g g}^{H^{*}}\left(m_{V V}\right)$. Therefore, the following correlation treatment of uncertainties is applied: the uncertainty on the signal $\mathrm{K}$-factor $\mathrm{K}^{H^{*}}\left(m_{V V}\right)$ is applied as a correlated uncertainty to $\mathrm{K}_{g g}^{H^{*}}\left(m_{V V}\right)$. The difference in quadrature between the uncertainty on $\mathrm{K}_{g g}^{H^{*}}\left(m_{V V}\right)$ and $\mathrm{K}^{H^{*}}\left(m_{V V}\right)$ is added as an uncorrelated uncertainty component only to $\mathrm{K}_{g g}^{H^{*}}\left(m_{V V}\right)$.

The interference between $g g \rightarrow H^{*} \rightarrow V V$ and $g g \rightarrow$ $V V$ is calculated at LO only. In Ref. [36], a soft-collinear approximation is used to calculate the cross-section for the sum of a heavy Higgs boson $(g g \rightarrow H \rightarrow W W)$ and its interference with the background. The uncertainty on this calculation is estimated to be about $10 \%$, which leads to about $30 \%$ uncertainty on the interference alone. Within the ansatz of using an unknown K-factor ratio between background and signal (see Eq. (5)), this additional uncertainty of roughly $30 \%$ on the interference term can be represented by an approximately $60 \%$ variation of the $\mathrm{K}$-factor ratio $\mathrm{R}_{H^{*}}^{B}$ for the background around the nominal value of 1.0. Therefore the variation of $\mathrm{R}_{H^{*}}^{B}$ from 0.5 to 2.0 should cover both the leading corrections and uncertainties for the interference and the background component taken individually. 
However, there is a large cancellation between the background and the negative interference at the expected $95 \%$ confidence level upper limit value of $\mu_{\text {off-shell }}$, shown in Tables 3 and 4. This leads to a large artificial cancellation in the uncertainties of the $g g \rightarrow Z Z$ background and the interference, when treated as correlated. To account for additional uncertainties on the interference component that are not covered by the soft-collinear approximation, the $30 \%$ uncertainty on the interference derived in Ref. [36] is applied to the interference component in addition to, and uncorrelated with, other uncertainties.

The systematic uncertainties associated with SHERPAbased re-weighting in $p_{\mathrm{T}}$ of the $V V$ system are assessed by varying the renormalisation, factorisation and resummation scales in SHERPA. The larger in value between the scale variations in SHERPA and $50 \%$ of the difference between SHERPA and gg2VV+ PYTHIA8 is assigned as the systematic uncertainty. This conservative approach is chosen to consider potential uncertainties not accounted for by the scale variations. The impact of the PDF uncertainties is found to be negligible.

\subsection{Systematic uncertainties on $q \bar{q} \rightarrow V V$}

The missing-higher-order and PDF uncertainties for the $q \bar{q} \rightarrow Z Z$ background, as a function of $m_{Z Z}$, are taken from Ref. [39], based on NLO $7 \mathrm{TeV}$ calculations using a fixed scale of $m_{Z}$. Slightly smaller systematic uncertainties are found for $8 \mathrm{TeV}$ using a dynamic scale of $m_{Z Z} / 2$, hence applying the uncertainties from Ref. [39] can be considered a conservative choice. Both the QCD scale uncertainty and the PDF uncertainty are 5-10\% for the highmass region used in this analysis. The NNLO calculation in Ref. [43] does not yield a significantly reduced QCD scale systematic uncertainty. An evaluation of the PDF uncertainty correlations shows that the $q \bar{q} \rightarrow Z Z$ background PDF uncertainties are anti-correlated with the PDF uncertainties for the $g g \rightarrow\left(H^{*} \rightarrow\right) Z Z$ process, and this is taken into account in the analysis. Acceptance uncertainties on the $q \bar{q} \rightarrow Z Z$ background are evaluated by comparing PYTHIA8 and HERWIG6 [59] samples and found to be negligible. The PDF, QCD scale, and EW correction uncertainties for the $q \bar{q} \rightarrow W Z$ process are considered in the same way as for the $q \bar{q} \rightarrow Z Z$ process. Both the QCD scale uncertainty and the PDF uncertainty are estimated to be $\sim 5-10 \%$ for the high-mass region used in this analysis.

Extrapolation uncertainties on the $q \bar{q} \rightarrow W W$ process in the $W W \rightarrow e v \mu \nu$ channel are evaluated using the method described in Ref. [58]. Uncertainties due to missing higher-order corrections are estimated by varying the renormalisation and factorisation scales independently by factors of one-half and two, keeping the ratio of the scales between one-half and two. Parton shower
Table 2 Uncertainties on the extrapolation of top-quark processes and $q \bar{q} \rightarrow W W$ from their respective CRs to the SR, and from the top CR to the $W W \mathrm{CR}$, from the parton shower and underlying event (UE/PS), from matching the matrix element to the UE/PS model (Gen), from the QCD renormalisation and factorisation scale (scale), and from the PDFs. These uncertainties are used in the $W W$ analysis and derived with the same methods as used in Ref. [58]

\begin{tabular}{lllll}
\hline & UE/PS (\%) & Gen. (\%) & Scale (\%) & PDF (\%) \\
\hline Top CR & 6.4 & 2.4 & 2.4 & 2.4 \\
$W W$ CR & 2.5 & 2.8 & 2.3 & 1.5 \\
\hline
\end{tabular}

and matrix-element uncertainties are estimated by comparing POWHEG-BOX+PYTHIA8 with POWHEG-BOX+ HERWIG6 and POWHEG-BOX+HERWIG6 with aMC@ NLO [37]+HERWIG6, respectively. PDF uncertainties are estimated by taking the largest difference between the nominal CT10 [60] and either the MSTW2008 [35] or the NNPDF2.1 [61] PDF set and adding this in quadrature with the CT10 error eigenvectors (following the procedure described in Ref. [62]). The extrapolation uncertainties from the $W W$ control region to the signal region are summarised in Table 2.

The EW corrections for the $q \bar{q} \rightarrow V V$ process described in Sect. 2.3 are strictly valid only for the $\mathrm{LO} \mathrm{QCD} q \bar{q} \rightarrow V V$ process above the diboson production threshold when both vector bosons are on shell. This is the case for all three analyses after final selections. The EW corrections are computed at LO QCD because the mixed QCD-EW corrections have not yet been calculated. In events with high QCD activity, an additional systematic uncertainty is considered by studying the variable $\rho=\left|\sum_{i} \vec{\ell}_{i, \mathrm{~T}}+\vec{E}_{\mathrm{T}}^{\text {miss }}\right| /\left(\sum_{i}\left|\vec{\ell}_{i, \mathrm{~T}}\right|+\left|\vec{E}_{\mathrm{T}}^{\text {miss }}\right|\right)$ introduced in Eq. (4.4) of Ref. [47] (here $\vec{\ell}_{T}$ represents the transverse momentum of the lepton $i$ from vector boson decays). A phase space region with $\rho<0.3$ is selected, where the NLO QCD event kinematics resembles the LO event kinematics in being dominated by recoiling vector bosons and therefore the corrections are applicable without additional uncertainty. For events with $\rho>0.3$ the correction is applied with a $100 \%$ systematic uncertainty to account for the missing mixed QCD-EW corrections which are expected to be of the same order of magnitude. The applied corrections are partial in that they include only virtual corrections, and do not include polarisation effects. The sum of both of these effects is estimated to be $\mathcal{O}(1 \%)$ [47] and is neglected in this analysis.

While the EW corrections and uncertainties directly affect the predicted size of the $q \bar{q} \rightarrow Z Z$ and $q \bar{q} \rightarrow W Z$ backgrounds in the $Z Z \rightarrow 4 \ell$ and $Z Z \rightarrow 2 \ell 2 v$ analyses, only the extrapolation of the $q \bar{q} \rightarrow W W$ background from the control region to the signal region is affected in the $W W \rightarrow e v \mu \nu$ analysis. 


\subsection{Systematic uncertainties on top-quark events}

Theory uncertainties on extrapolating top-quark processes from the control region to the signal region in the $W W \rightarrow$ ev $\mu \nu$ channel are also evaluated using methods similar to those of Ref. [58]. For the evaluation of the extrapolation uncertainties, the signal region requirements are relaxed to increase the sample size; the region is extended down to $\mathrm{R}_{8}>160 \mathrm{GeV}$ and the $\Delta \eta_{\ell \ell}$ requirement is dropped. The extra uncertainty from this extension is checked in a separate sample with at least one $b$-tagged jet, again defined so as to reduce the statistical uncertainties, which is simultaneously re-weighted in $\Delta \eta_{\ell \ell}$ and $\mathrm{R}_{8}$ to match the $b$-vetoed region. With this $b$-tagged sample, the extra uncertainty from the removal of the $\Delta \eta_{\ell \ell}$ requirement, and from extending the range in $\mathrm{R}_{8}$, is found to be $3.5 \%$.

The method described in Sect. 6.2 is used to evaluate the systematic uncertainties on top-quark processes. Since the extended signal region covers the $W W \mathrm{CR}$, the same systematic uncertainties are valid for the extrapolation from the top $\mathrm{CR}$ to the $W W \mathrm{CR}$. These uncertainties, summarised in Table 2, are applied to both $t \bar{t}$ and single-top processes, which make up approximately $22 \%$ of the top background in the signal region. A $20 \%$ uncertainty is assigned to the single-top processes in order to take into account the uncertainty on the single-top fraction; the impact on the result is negligible.

\subsection{Experimental systematic uncertainties}

For the $Z Z \rightarrow 4 \ell$ analysis, the same sources of experimental uncertainty as in Ref. [55] are evaluated. In the off-shell Higgs boson region, the leptons come from the decay of onshell $Z$ bosons; hence the lepton-related systematic uncertainties are small compared to those for the leptons from on-shell Higgs boson production. The leading, but still very small, experimental systematic uncertainties are due to the electron and muon reconstruction efficiency uncertainties.

Similarly, for the $2 \ell 2 v$ channel, the same sources of experimental uncertainty as in Ref. [20] are evaluated. The electron energy scale, electron identification efficiency, muon reconstruction efficiency, jet energy scale, and systematic uncertainties from the data-driven $Z$ background estimates are the main sources of the experimental systematic uncertainties. These experimental uncertainties affect the expected sensi-

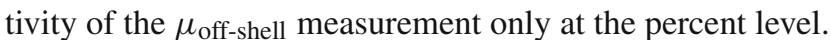

Finally, for the $W W \rightarrow e v \mu \nu$ channel, the same sources of experimental uncertainty as in Ref. [58] are evaluated. The uncertainty on the electron energy scale, followed by the uncertainty on the rate for mis-tagged light-flavour jets as $b$-jets, and the uncertainty on the jet energy scale and resolution, are the dominant experimental sources of uncertainty.
The remaining experimental sources are significantly smaller than the theoretical uncertainties.

The uncertainty on the integrated luminosity is $2.8 \%$. It is derived, following the same methodology as that detailed in Ref. [63], from a preliminary calibration of the luminosity scale derived from beam-separation scans performed in November 2012.

\section{Results}

In this section the results for the $Z Z \rightarrow 4 \ell, Z Z \rightarrow 2 \ell 2 v$ and $W W \rightarrow e \nu \mu \nu$ analyses are presented and translated into limits on the off-shell signal strength $\mu_{\text {off-shell for the individ- }}$ ual analyses and for the combination of all three channels. In a second step, the off-shell analyses are combined with the on-shell $Z Z^{*} \rightarrow 4 \ell$ [55] and $W W^{*} \rightarrow \ell v \ell v$ [58] analyses based on the $8 \mathrm{TeV}$ data taken in 2012. In combining the $Z Z$ and $W W$ channels it is assumed that the ratio of the $Z Z$ cross-section $\sigma^{g g \rightarrow H^{(*)}} \rightarrow Z Z(\hat{s})$ to the $W W$ cross-section $\sigma^{g g \rightarrow H^{(*)}} \rightarrow W W(\hat{s})$ (and similarly for $\mathrm{VBF}$ ) is as predicted in the SM for both the on- and off-shell processes.

Two different off-shell combinations are presented based on different assumptions. First, a single off-shell signal strength parameter is applied for all production modes. This is equivalent to assuming that the ratio of the off-shell production rates via the process $g g \rightarrow H$ to those via the VBF process are as predicted in the SM. In a second combination, only the off-shell signal strength of the $g g \rightarrow H^{*} \rightarrow V V$ production process is considered while the VBF production process is fixed to the SM prediction. In this case the combined signal strength $\mu_{\text {off-shell }}^{g g \rightarrow H^{*}} \rightarrow V V$ can be interpreted as a constraint on the off-shell coupling strength $\kappa_{g}$,off-shell associated with the $g g \rightarrow H^{*}$ production mode.

The combination with the on-shell analyses is also performed under two assumptions that correspond to different interpretations of the results. The first is performed using different signal strengths for the $g g \rightarrow H^{(*)}$ and the VBF production modes. ${ }^{6}$ The parameter of interest is described by the ratio of the off-shell to the on-shell signal strengths, which can be interpreted as the Higgs boson total width normalised to its SM prediction: $\mu_{\text {off-shell }} / \mu_{\text {on-shell }}=\Gamma_{H} / \Gamma_{H}^{\mathrm{SM}}$. This interpretation requires that the off- and on-shell couplings are the same for both $g g \rightarrow H^{(*)}$ and VBF production modes (i.e., $\kappa_{g, \text { on-shell }}=\kappa_{g \text {,off-shell }}$ and $\kappa_{V \text {,on-shell }}=\kappa_{V \text {,off-shell }}{ }^{7}$ ). In a second combination, the coupling scale factors $\kappa_{V}=$

\footnotetext{
${ }^{6}$ In all results the signal strength for $V H$ associated production is assumed to scale with VBF production while the $b \bar{b} H$ and $t \bar{t} H$ processes scale with the $g g \rightarrow H$ process. These additional production modes are expected to give negligible contributions to the off-shell measurements, but have small contributions to the on-shell signal yields.

7 To set an upper limit, the assumption in Eq. (3), and the equivalent assumption for the VBF production mode, is sufficient.
} 
$\kappa_{V \text {,on-shell }}=\kappa_{V \text {,off-shell associated with the on- and off-shell }}$ VBF production and the $H^{(*)} \rightarrow V V$ decay, are assumed to be the same and fitted to the data (profiled). In this case the parameter of interest, $R_{g g}=\mu_{\text {off-shell }}^{g g \rightarrow H^{*}} / \mu_{\text {on-shell }}^{g g \rightarrow H}$, can be interpreted as the ratio of the off-shell to the on-shell gluon couplings: $R_{g g}=\kappa_{g \text {, off-shell }}^{2} / \kappa_{g \text {,on-shell }}^{2}$. This also assumes that the total width is equal to the SM prediction.

In the $Z Z \rightarrow 4 \ell$ channel, a binned maximum-likelihood fit to the ME-based discriminant distribution is performed to extract the limits on the off-shell Higgs boson signal strength. The fit model accounts for signal and background processes, including $g g \rightarrow\left(H^{*} \rightarrow\right) Z Z, \operatorname{VBF}\left(H^{*} \rightarrow\right) Z Z$ and $q \bar{q} \rightarrow Z Z$. The probability density functions (pdf) of the signal-related processes $g g \rightarrow\left(H^{*} \rightarrow\right) Z Z$ and VBF $\left(H^{*} \rightarrow\right) Z Z$ are parameterised as a function of both the offshell Higgs boson signal strength $\mu_{\text {off-shell }}$ and the unknown background $\mathrm{K}$-factor ratio $\mathrm{R}_{H^{*}}^{B}$ as given in Eqs. (15) and (19). Normalisation and shape systematic uncertainties on the signal and background processes are taken into account as described in Sect. 6.1, with correlations between different components and processes as indicated therein.

In the $Z Z \rightarrow 2 \ell 2 v$ channel, a similar maximumlikelihood fit to the transverse mass $\left(m_{\mathrm{T}}^{Z Z}\right)$ is performed, comparing the event yield in the signal-enriched region in data with the predictions. The fit model accounts for the signal and all background processes mentioned in Table 1. The modelling of the dominant signal and background processes is the same as in the $Z Z \rightarrow 4 \ell$ channel.

In the $W W \rightarrow e v \mu \nu$ channel, a maximum-likelihood fit is performed using the event yields in the signal region and the two control regions. As in the $Z Z$ channels, the fit model accounts for the parameterised signal and all background processes mentioned in Sect. 5.2. Unconstrained strength parameters common among fit regions for the $q \bar{q} \rightarrow W W$ and topquark processes allow the control regions to constrain the predicted event yields in the signal region.

The likelihood is a function of a parameter of interest $\mu$ and nuisance parameters $\vec{\theta}$. Hypothesis testing and confidence intervals are based on the profile likelihood ratio [64]. The parameters of interest are different in the various tests, while the remaining parameters are profiled. Hypothesised values for a parameter of interest $\mu$ are tested with a statistic

$\Lambda(\mu)=\frac{L(\mu, \hat{\overrightarrow{\hat{\theta}}}(\mu))}{L(\hat{\mu}, \hat{\vec{\theta}})}$,

where the single circumflex denotes the unconditional maximum-likelihood estimate of a parameter and the double circumflex [e.g. $\hat{\vec{\theta}}(\mu)$ ] denotes the conditional maximumlikelihood estimate (e.g. of $\vec{\theta}$ ) for given fixed values of $\mu$. This test statistic extracts the information on the parameters of interest from the full likelihood function.
All $95 \%$ confidence level (CL) upper limits are derived using the $C L_{S}$ method [65], based on the following ratio of one-sided $p$-values: $C L_{S}(\mu)=p_{\mu} /\left(1-p_{1}\right)$ where $p_{\mu}$ is the

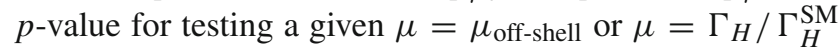
(the non-SM hypothesis) and $p_{1}$ is the $p$-value derived from the same test statistic under the SM hypothesis of $\mu_{\text {off-shell }}=1$ in the first case and $\Gamma_{H} / \Gamma_{H}^{\mathrm{SM}}=\mu_{\text {on-shell }}=1$ in the second case. ${ }^{8}$ The $95 \% C L_{s}$ upper limit is found by solving for $C L_{s}\left(\mu^{95 \%}\right)=5 \%$. Values $\mu>\mu^{95 \%}$ are regarded as excluded at $95 \%$ CL. A detailed description of the implementation of the $C L_{s}$ procedure can be found in Ref. [66].

The results presented in this paper rely on the asymptotic approximation [64] for the test statistic $\Lambda(\mu)$. This approximation was cross-checked with Monte Carlo ensemble tests that confirm its validity in the range of the parameters for which the $95 \%$ CL limits are derived. Deviations appear close to the boundary of $\mu_{\text {off-shell }} \geq 0$ imposed by Eq. (5) and hence the $1 \sigma$ uncertainties can only be seen as approximate.

While the final $95 \%$ CL limits are given as a function of the unknown background $\mathrm{K}$-factor ratio $\mathrm{R}_{H^{*}}^{B}$, comparisons between the data and the MC predictions, and values in other figures and tables, are given assuming $\mathrm{R}_{H^{*}}^{B}=1$.

\subsection{Results of the individual off-shell analyses}

The scan of the negative $\log$-likelihood, $-2 \ln \Lambda$, as a func-

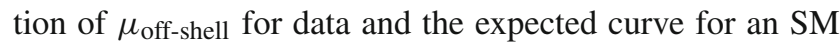
Higgs boson for the three individual off-shell analyses is illustrated in Fig. 6. The observed and expected $95 \% \mathrm{CL}$

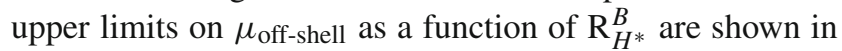
Fig. 7 and are summarised in Table 3. The $Z Z \rightarrow 4 \ell$ and $Z Z \rightarrow 2 \ell 2 v$ analysis have a very similar expected sensitivity. The $Z Z \rightarrow 4 \ell$ analysis is statistics limited, while the sensitivity in the $Z Z \rightarrow 2 \ell 2 v$ analysis is significantly reduced by the theoretical systematic uncertainties as can be seen in Fig. 6. The similar expected $C L_{S}$ limits for the two channels for $\mathrm{R}_{H^{*}}^{B}=0.5$ and 1.0 in Table 3 is a coincidence, caused by the different statistical and systematic uncertainty components.

The typical off-shell mass scales tested by the analyses are in the range $400 \mathrm{GeV}<m_{V V}<1000 \mathrm{GeV}$, with a small fraction of the expected $H^{*} \rightarrow W W$ signal extending to substantially higher mass scales. ${ }^{9}$ This is illustrated in Fig. 8, which shows the generated $m_{V V}$ mass for the $g g \rightarrow H^{*} \rightarrow$ $V V$ and the VBF $H^{*} \rightarrow V V$ signal processes weighted by the expected $\mathrm{S} / \mathrm{B}$ ratio in each bin of the final discriminant

\footnotetext{
${ }^{8}$ In the context of this analysis the alternative hypothesis is given by the SM value(s) for all relevant parameters of the fit model.

${ }^{9}$ While the $H^{*} \rightarrow Z Z$ analysis includes a selection cut to limit the mass range to $m_{Z Z} \lesssim 1000 \mathrm{GeV}$, no such cut can be efficiently implemented for the $H^{*} \rightarrow W W$ analysis due to the poor mass resolution.
} 

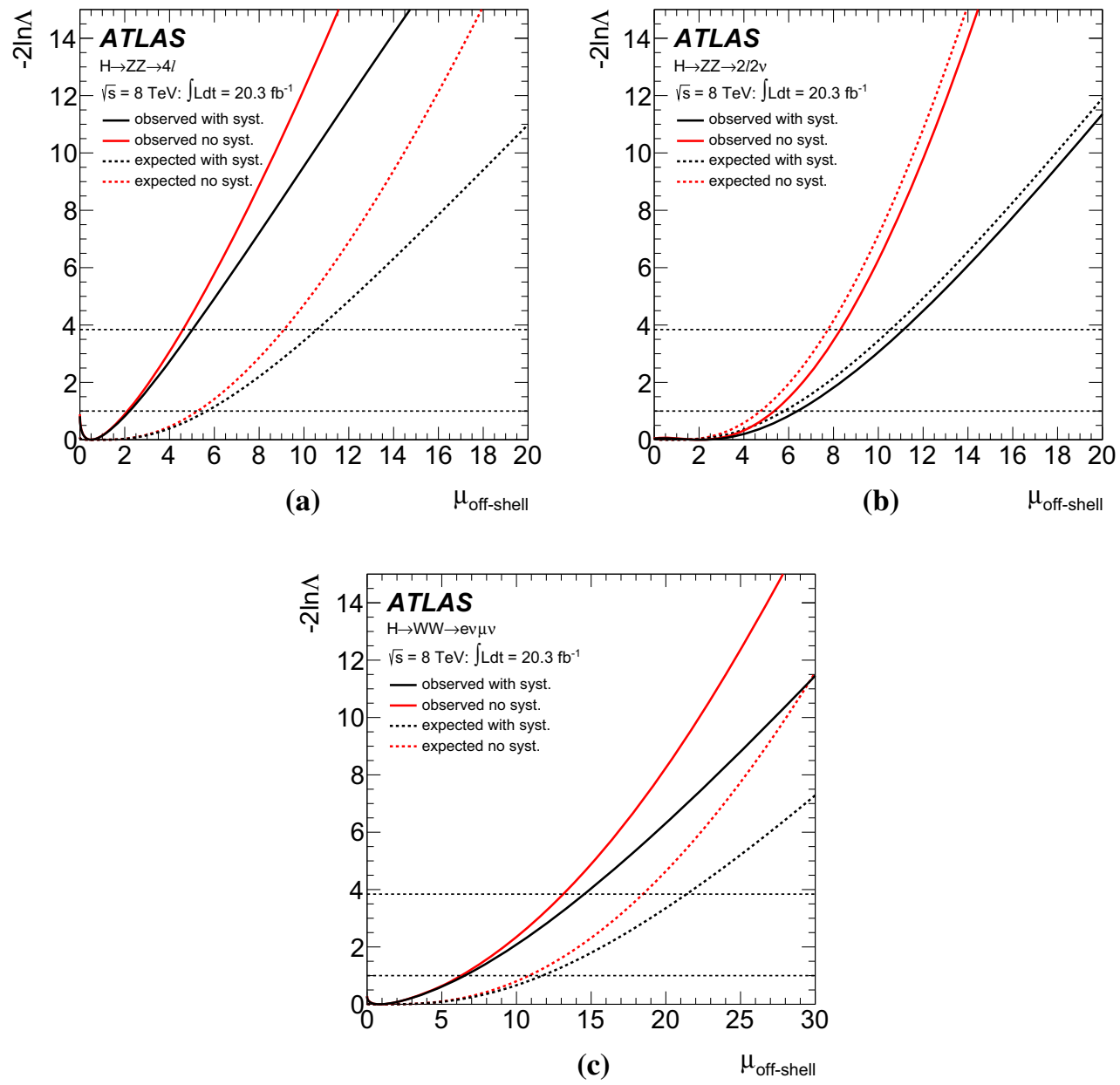

Fig. 6 Scan of the negative $\log$-likelihood, $-2 \ln \Lambda$, as a function of

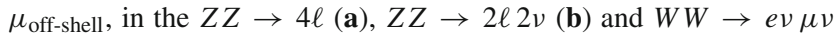
(c) channels. The black solid (dashed) line represents the observed (expected) value including all systematic uncertainties, while the red

for the $Z Z \rightarrow 4 \ell$ and $Z Z \rightarrow 2 \ell 2 v$ analyses and for all signal events in the signal region for the $W W \rightarrow e v \mu \nu$ analysis.

\subsection{Combination of the off-shell $Z Z$ and $W W$ analyses}

The analyses described in the previous sections are combined to obtain a limit on $\mu_{\text {off-shell }}$. In combining the off-shell results the main systematic uncertainties related to the theory uncertainties on the $g g \rightarrow\left(H^{*} \rightarrow\right) V V$ (including signal and interference contributions) and $q \bar{q} \rightarrow V V$ processes are treated as correlated between the different channels. The same K-factor ratio $\mathrm{R}_{H^{*}}^{B}$ is assumed for the $g g \rightarrow Z Z$ and $g g \rightarrow W W$ backgrounds. Where appropriate, the experimental systematic uncertainties are also treated as correlated. However, they are found to have a very small impact on the final combined limit.

The limits on $\mu_{\text {off-shell }}$ are obtained under two different assumptions: solid (dashed) line is for the observed (expected) value without systematic uncertainties. A relative $g g \rightarrow V V$ background K-factor of $\mathrm{R}_{H^{*}}^{B}=1$ is assumed in these figures

- Determination of the signal strength $\mu_{\text {off-shell }}$ when fixing the ratio of the signal strength in $g g \rightarrow H^{*}$ and VBF to the SM prediction, namely $\mu_{\text {off-shell }}^{g g \rightarrow H^{*}} / \mu_{\text {off-shell }}^{V B F}=1$.

- Determination of the signal strength $\mu_{\text {off-shell }}^{g g \rightarrow H^{*} \rightarrow V V}$ when fixing the VBF off-shell signal strength to the SM prediction, i.e. $\mu_{\mathrm{off}-\text { shell }}^{\mathrm{VBF} H^{*} \rightarrow V V}=1$.

The scan of the negative $\log$-likelihood, $-2 \ln \Lambda$, as a function of $\mu_{\text {off-shell }}$ for data and the expected curve for an SM Higgs boson for the two cases above are shown in Fig. 9.

The limits on $\mu_{\text {off-shell }}$ and $\mu_{\text {off-shell }}^{g g \rightarrow H^{*}}$ are computed with the $C L_{s}$ method, assuming for the alternative hypothesis that all the off-shell rates are at their SM predictions. They are derived as a function of the $g g \rightarrow V V$ background $\mathrm{K}$-factor ratio $\mathrm{R}_{H^{*}}^{B}$. These results are reported in Table 4 and shown in Fig. 10, assuming either one common scale factor for both the $g g \rightarrow H^{*}$ and VBF processes or using a scale factor for the $g g \rightarrow H^{*}$ process and fixing the VBF production to the SM prediction. 


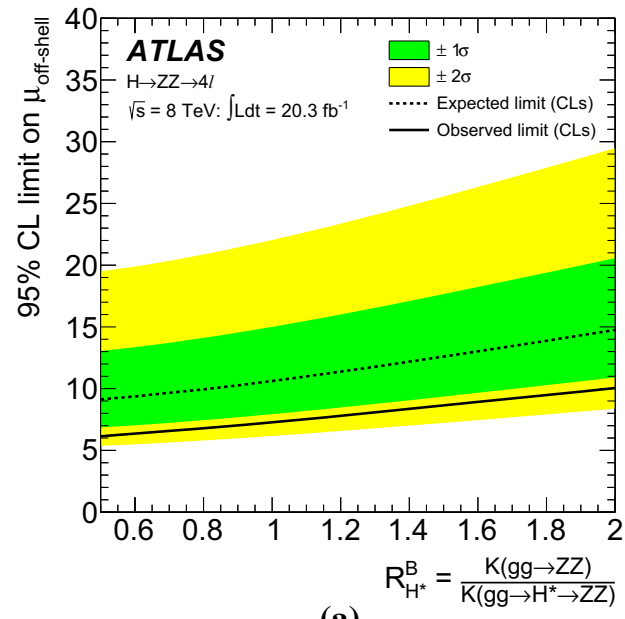

(a)

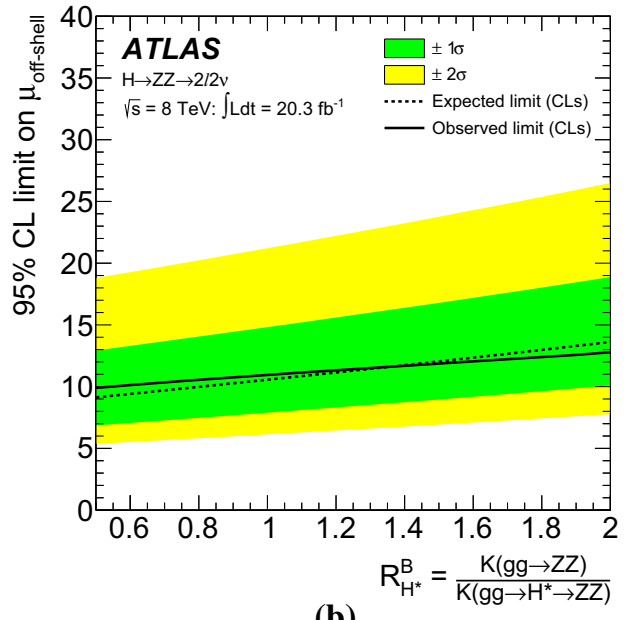

(b)

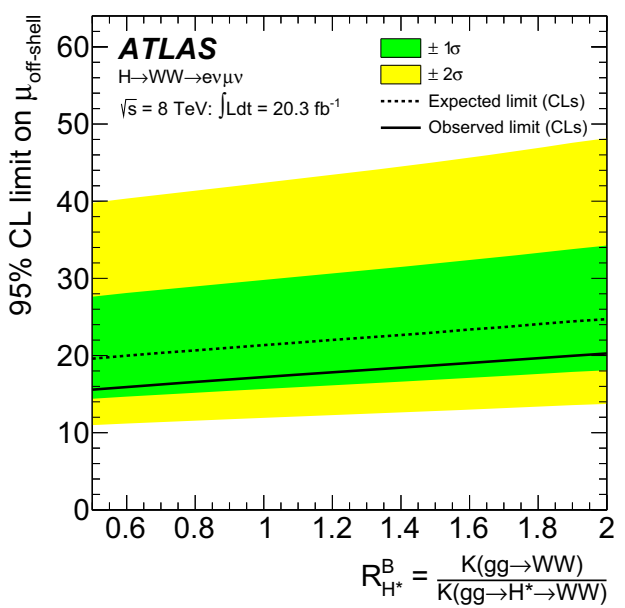

(c)

Fig. 7 The observed and expected $95 \%$ CL upper limit on $\mu_{\text {off-shell }}$ as a function of $\mathrm{R}_{H^{*}}^{B}$, for the $Z Z \rightarrow 4 \ell(\mathbf{a}), Z Z \rightarrow 2 \ell 2 v(\mathbf{b})$ and $W W \rightarrow e v \mu \nu$ (c) channels. The upper limits are evaluated using the
$C L_{s}$ method, with the alternative hypothesis $\mu_{\text {off-shell }}=1$. The green (yellow) bands represent the $68 \%(95 \%)$ confidence intervals for the $C L_{s}$ expected limit

Table 3 The observed and expected $95 \%$ CL upper limits on $\mu_{\text {off-shell }}$ within the range of $0.5<\mathrm{R}_{H^{*}}^{B}<2.0$. The bold numbers correspond to the limit assuming $\mathrm{R}_{H^{*}}^{B}=1$. The upper limits are evaluated using the $C L_{s}$ method, with the alternative hypothesis $\mu_{\mathrm{off}-\text { shell }}=1$

\begin{tabular}{|c|c|c|c|c|c|c|}
\hline \multirow[t]{2}{*}{$\mathrm{R}_{H^{*}}^{B}$} & \multicolumn{3}{|c|}{ Observed } & \multicolumn{3}{|c|}{ Median expected } \\
\hline & 0.5 & 1.0 & 2.0 & 0.5 & 1.0 & 2.0 \\
\hline$Z Z \rightarrow 4 \ell$ analysis & 6.1 & 7.3 & 10.0 & 9.1 & 10.6 & 14.8 \\
\hline$Z Z \rightarrow 2 \ell 2 v$ analysis & 9.9 & 11.0 & 12.8 & 9.1 & 10.6 & 13.6 \\
\hline$W W \rightarrow e \nu \mu \nu$ analysis & 15.6 & 17.2 & 20.3 & 19.6 & 21.3 & 24.7 \\
\hline
\end{tabular}

The impact of the various systematic uncertainties on the combined expected limit in the off-shell fit are listed in Table 5 when fixing the ratio of the signal strength in $g g \rightarrow H^{*}$ and VBF to the SM prediction. The values in this table were derived by fixing all the nuisance parameters associated with the systematic uncertainties to the values derived from the SM-conditional fit to the data, with the exception of the one under study.
7.3 Combination of the off-shell and on-shell $Z Z$ and $W W$ analyses

In this section, the off-shell results reported above are combined with the on-shell $H \rightarrow Z Z^{*} \rightarrow 4 \ell$ [55] and $H \rightarrow$ $W W^{*} \rightarrow \ell \nu \ell \nu$ [58] analyses based on the $8 \mathrm{TeV}$ data taken in 2012. In these analyses a Higgs boson mass value of $125.36 \mathrm{GeV}$ [11] is assumed. For the on-shell $Z Z$ and $W W$ 
combination the main common sources of theoretical and experimental systematic uncertainties are treated as correlated [3].

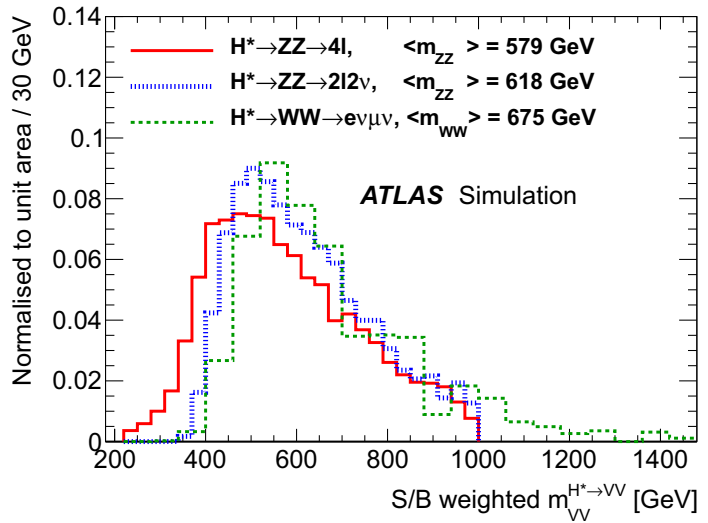

Fig. 8 Normalised distribution of the generated mass $m_{V V}$ for the $g g \rightarrow H^{*} \rightarrow V V$ and the VBF $H^{*} \rightarrow V V$ signal processes weighted by the expected $\mathrm{S} / \mathrm{B}$ ratio in each bin of the final discriminant for the $Z Z \rightarrow 4 \ell$ and $Z Z \rightarrow 2 \ell 2 v$ analyses and for all events in the signal region for the $W W \rightarrow e v \mu \nu$ analysis

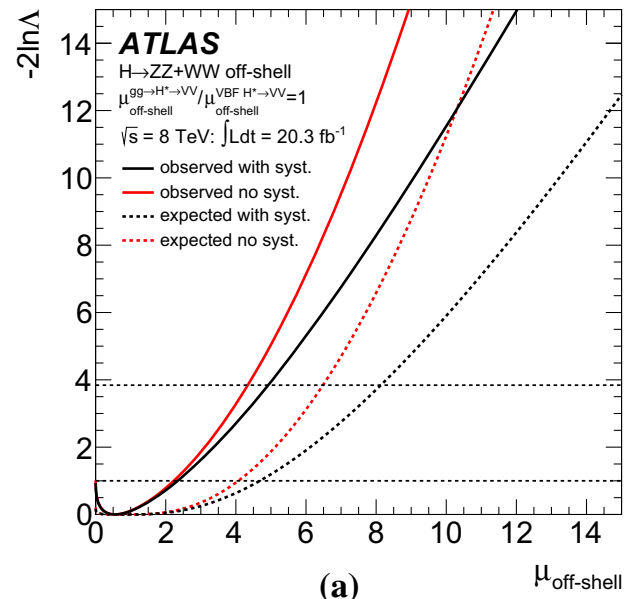

Fig. 9 Scan of the negative $\log$-likelihood, $-2 \ln \Lambda$, as a function of $\mu_{\text {off-shell }}$, for the combined $Z Z$ and $W W$ analyses. a Com-

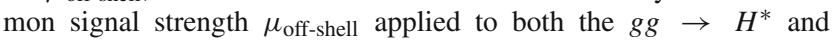
VBF processes. The ratio of the $g g \rightarrow H^{*}$ and VBF processes is assumed to be as in the SM. b Signal strength $\mu_{\text {off-shell }}^{g g \rightarrow H^{*} \rightarrow V}$ for the $g g \rightarrow H^{*} \rightarrow V V$ process. The production rate for the $\mathrm{VBF}$

Table 4 The observed and expected $95 \%$ CL upper limits on $\mu_{\text {off-shell }}$ and $\mu_{\text {off-shell }}^{g g \rightarrow} \rightarrow H^{*} \rightarrow$ within the range of $0.5<\mathrm{R}_{H^{*}}^{B}<2$ for the combined $Z Z$ and $W W$ analyses. Results are shown for two hypotheses, which
The uncertainties from the impact of higher-order QCD corrections on the $g g \rightarrow H^{(*)}$ and $q q \rightarrow V V$ processes are considered correlated between the on-shell and off-shell measurements. The PDF uncertainties are treated as uncorrelated between on-shell and off-shell analyses. The correlations between the PDF uncertainties for the on-shell and off-shell analyses are expected to be small with the exception of the ones for the $q \bar{q} \rightarrow V V$ process, which have negligible impact on the on-shell results.

In addition to the main theoretical uncertainties, the common experimental systematic uncertainties are treated as correlated.

The results reported in the following are based on two different assumptions:

- Determination of $\Gamma_{H} / \Gamma_{H}^{S M}$ when profiling the coupling scale factors $\kappa_{g}$ and $\kappa_{V}$ associated with the on- and offshell $g g \rightarrow H^{(*)}$ and VBF production and the $H^{(*)} \rightarrow$ $V V$ decay, assuming $\kappa_{g}=\kappa_{g \text {,on-shell }}=\kappa_{g \text {,off-shell and }}$ $\kappa_{V}=\kappa_{V \text {,on-shell }}=\kappa_{V \text {,off-shell. }}$

- Determination of $R_{g g}=\kappa_{g \text {,off-shell }}^{2} / \kappa_{g \text {,on-shell }}^{2}$ when profiling the coupling scale factor $\kappa_{V}=\kappa_{V \text {, on-shell }}=$

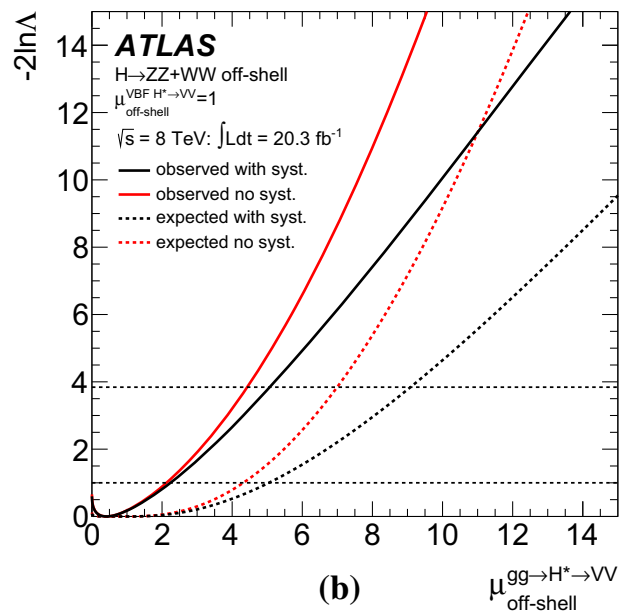

off-shell process is fixed to the SM prediction. The black solid (dashed) line represents the observed (expected) value including all systematic uncertainties, while the red solid (dashed) line is for the observed (expected) value without systematic uncertainties. A relative $g g \rightarrow V V$ background $\mathrm{K}$-factor of $\mathrm{R}_{H^{*}}^{B}=1$ is assumed in these figures

are defined in the assumption column. The bold numbers correspond to the limit assuming $\mathrm{R}_{H^{*}}^{B}=1$. The upper limits are evaluated using the $C L_{S}$ method, with the alternative hypothesis $\mu_{\text {off-shell }}=1$

\begin{tabular}{|c|c|c|c|c|c|c|c|}
\hline \multirow[t]{2}{*}{$\mathrm{R}_{H^{*}}^{B}$} & \multicolumn{3}{|c|}{ Observed } & \multicolumn{3}{|c|}{ Median expected } & \multirow[t]{2}{*}{ Assumption } \\
\hline & 0.5 & 1.0 & 2.0 & 0.5 & 1.0 & 2.0 & \\
\hline$\mu_{\text {off-shell }}$ & 5.1 & 6.2 & 8.6 & 6.7 & 8.1 & 11.0 & $\mu_{\text {off-shell }}^{g g \rightarrow H^{*}} / \mu_{\text {off-shell }}^{V B F}=1$ \\
\hline$\mu_{\text {off-shell }}^{g g \rightarrow H^{*}} \rightarrow V V$ & 5.3 & 6.7 & 9.8 & 7.3 & 9.1 & 13.0 & $\mu_{\text {off-shell }}^{\mathrm{VBF} H^{*}} \rightarrow^{V V}=1$ \\
\hline
\end{tabular}




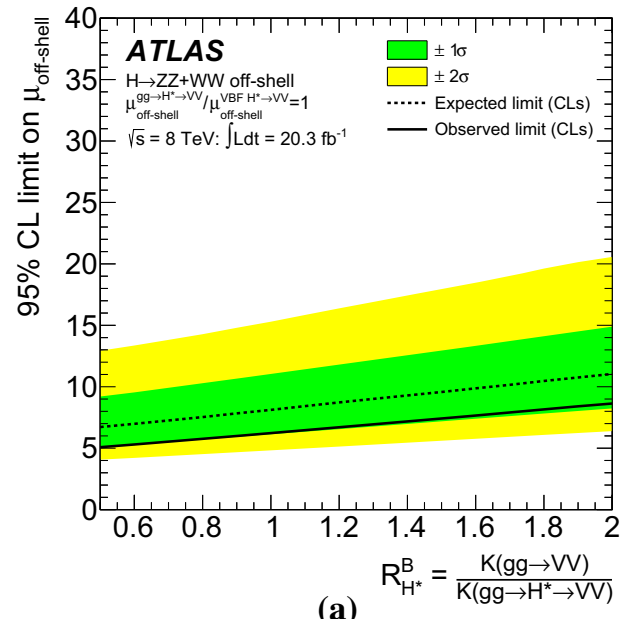

(a)

Fig. 10 The observed and expected combined $95 \%$ CL upper limit on $\mu_{\text {off-shell }}$ as a function of $\mathrm{R}_{H^{*}}^{B}$ for the combined $Z Z$ and $W W$ analyses. The upper limits are calculated using the $C L_{S}$ method, with the SM as the alternative hypothesis. a Limit on the common signal strength $\mu_{\text {off-shell }}$ applied to both the $g g \rightarrow H^{*}$ and VBF processes. The ratio

Table 5 The expected $95 \%$ CL upper limit on $\mu_{\text {off-shell }}$ for the combined $Z Z$ and $W W$ analyses, with a ranked listing of each systematic uncertainty individually, comparing with no systematic uncertainty or all systematic uncertainties. The upper limits are evaluated using the $C L_{s}$ method, assuming $\mathrm{R}_{H^{*}}^{B}=1$. The ratio of the $g g \rightarrow H^{*}$ and $\mathrm{VBF}$ processes is assumed to be as expected in the SM

\begin{tabular}{ll}
\hline Systematic uncertainty & $95 \%$ CL lim. $\left(C L_{s}\right)$ on $\mu_{\text {off }- \text { shell }}$ \\
\hline Interference $g g \rightarrow\left(H^{*} \rightarrow\right) V V$ & 7.2 \\
QCD scale $\mathrm{K}^{H^{*}}\left(m_{V V}\right)$ (correlated & 7.1 \\
$\quad$ component) & \\
PDF $q \bar{q} \rightarrow V V$ and & 6.7 \\
$g g \rightarrow\left(H^{*} \rightarrow\right) V V$ & \\
QCD scale $q \bar{q} \rightarrow V V$ & 6.7 \\
Luminosity & 6.6 \\
Drell-Yan background & 6.6 \\
QCD scale $\mathrm{K}_{g g}^{H^{*}}\left(m_{V V}\right)$ & 6.5 \\
$\quad$ uncorrelated component) & \\
Remaining systematic & 6.5 \\
uncertainties & 8.1 \\
All systematic uncertainties & 6.5 \\
No systematic uncertainties &
\end{tabular}

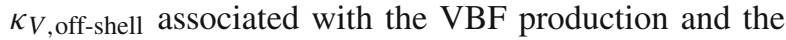
$H^{(*)} \rightarrow V V$ decay. The ratio $\Gamma_{H} / \Gamma_{H}^{\mathrm{SM}}=1$ is fixed to the SM prediction. The parameter $R_{g g}$ is sensitive to possible modifications of the gluon couplings in the highmass range with respect to the on-shell value.

The negative log-likelihood scans for the above-defined fitting configurations as well as the combined upper limit at $95 \% \mathrm{CL}$ on $\Gamma_{H} / \Gamma_{H}^{\mathrm{SM}}$ and $R_{g g}$ are illustrated in Figs. 11 and

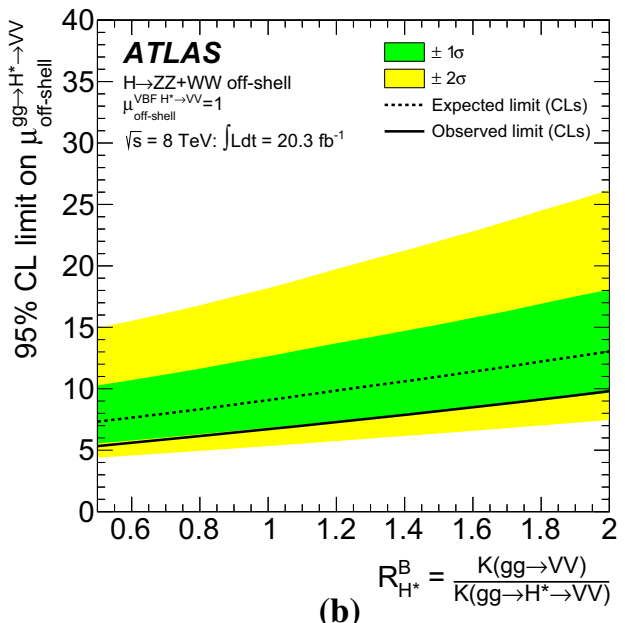

of the $g g \rightarrow H^{*}$ and VBF processes is assumed to be as in the SM. b Limit on the signal strength $\mu_{\text {off-shell }}^{g g \rightarrow H^{*}}$ for the $g g \rightarrow H^{*} \rightarrow V V$ process. The production rate for the VBF off-shell process is fixed to the SM prediction. The green (yellow) bands represent the $68 \%(95 \%)$ confidence intervals for the $C L_{s}$ expected limit

12 and the corresponding limits are listed in Table 6. The limits are all computed with the $C L_{s}$ method, taking the SM values as the alternative hypothesis.

The limit on $\Gamma_{H} / \Gamma_{H}^{\mathrm{SM}}$ can be translated into a limit on the total width of the Higgs boson under the assumptions reported above, out of which the most important is that the relevant Higgs boson coupling scale factors are independent of the energy scale of the Higgs boson production. Assuming a value of $\mathrm{R}_{H^{*}}^{B}=1$, this translates into an observed (expected) $95 \%$ CL upper limit on the Higgs boson total width of 22.7 (33.0) MeV. ${ }^{10}$

\section{Conclusion}

The measurement of the $Z Z$ and $W W$ final states in the mass range above the $2 m_{Z}$ and $2 m_{W}$ thresholds provides a unique opportunity to measure the off-shell coupling strengths of the observed Higgs boson. In this paper constraints on the off-shell Higgs boson signal strengths in the $Z Z \rightarrow 4 \ell$, $Z Z \rightarrow 2 \ell 2 v$ and $W W \rightarrow e v \mu v$ final states and their combination are presented. The result is based on $p p$ collision data collected by the ATLAS experiment at the LHC, corresponding to an integrated luminosity of $20.3 \mathrm{fb}^{-1}$ at a collision energy of $\sqrt{s}=8 \mathrm{TeV}$.

Using the $C L_{s}$ method, the observed $95 \%$ confidence level (CL) upper limit on the off-shell signal strength is in the range 5.1-8.6, with an expected range of 6.7-11.0. In each

10 The value of the SM Higgs boson width of $4.12 \mathrm{MeV}$ at a mass of $125.4 \mathrm{GeV}$ [23] is used to convert the limit $\Gamma_{H} / \Gamma_{H}^{\mathrm{SM}}$ into the total width limit. 


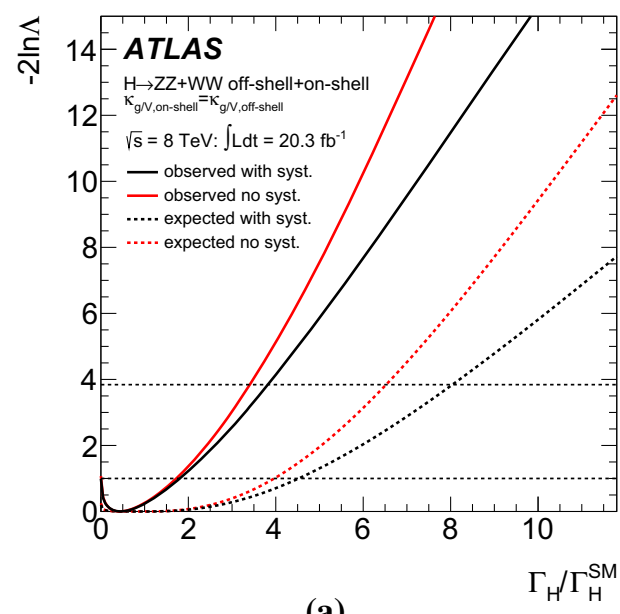

(a)

Fig. 11 a Scan of the negative log-likelihood as a function of $\Gamma_{H} / \Gamma_{H}^{S M}$ when profiling the coupling scale factors $\kappa_{g}$ and $\kappa_{V}$ associated with the on- and off-shell $g g \rightarrow H^{(*)}$ and VBF production and the $H^{(*)} \rightarrow V V$ decay. The black solid (dashed) line represents the observed (expected) value including all systematic uncertainties, while the red solid (dashed) line is for the observed (expected) value without systematic

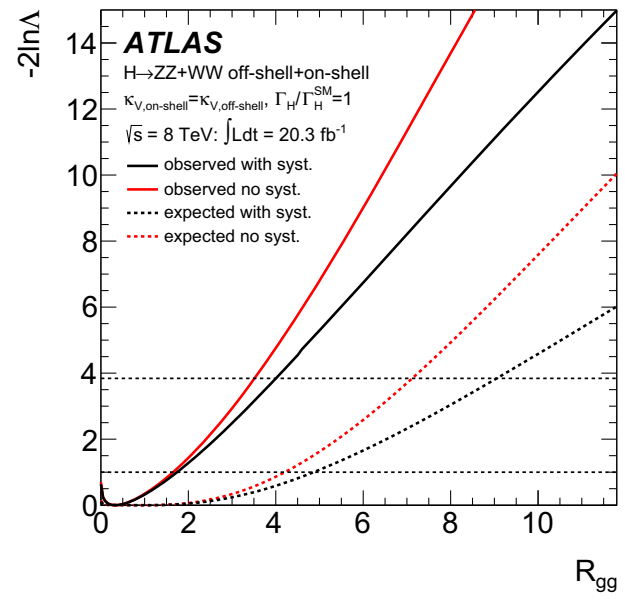

(a)

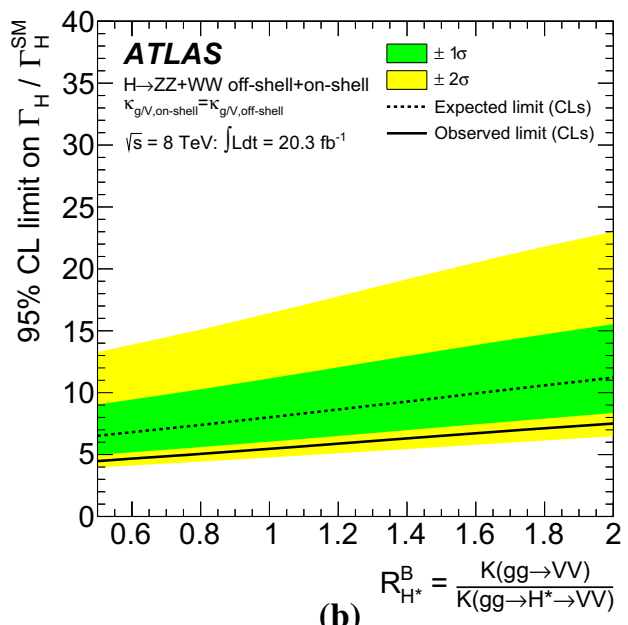

(b)

uncertainties. b Observed and expected combined $95 \%$ CL upper limit on $\Gamma_{H} / \Gamma_{H}^{\mathrm{SM}}$ as a function of $\mathrm{R}_{H^{*}}^{B}$ under the same assumption as $\mathbf{a}$. The upper limits are calculated from the $C L_{S}$ method, with the SM values as the alternative hypothesis. The green (yellow) bands represent the $68 \%(95 \%)$ confidence intervals for the $C L_{s}$ expected limit

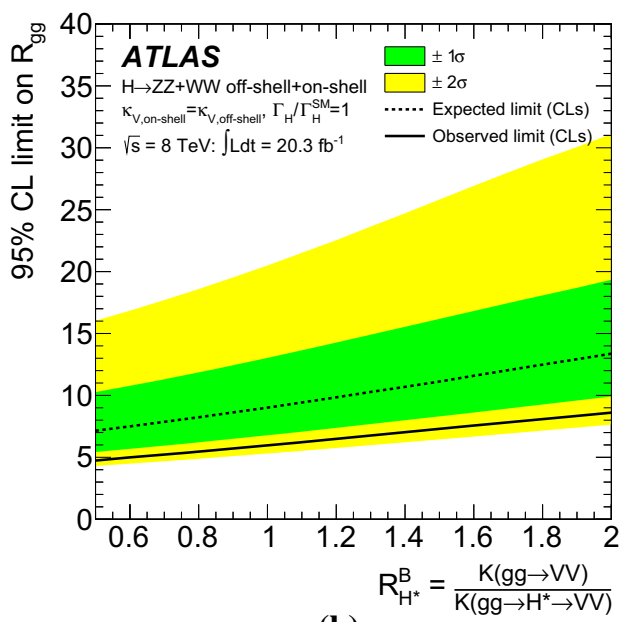

(b)

b Observed and expected combined $95 \%$ CL upper limit on $R_{g g}$ as a function of $\mathrm{R}_{H^{*}}^{B}$ under the same assumption as $\mathbf{a}$. The upper limits are calculated from the $C L_{s}$ method, with the SM values as the alternative hypothesis. The green (yellow) bands represent the $68 \%(95 \%)$ confidence intervals for the $C L_{S}$ expected limit including all systematic uncertainties, while the red solid (dashed) line is for the observed (expected) value without systematic uncertainties.

Table 6 Observed and expected $95 \%$ CL upper limits on $\Gamma_{H} / \Gamma_{H}^{\mathrm{SM}}$ and $R_{g g}$ for the combined on- and off-shell $Z Z$ and $W W$ analyses. Results are shown for two hypotheses, which are defined in the assumption column. $\mathrm{R}_{H^{*}}^{B}$ is within the range $0.5<\mathrm{R}_{H^{*}}^{B}<2$

\begin{tabular}{|c|c|c|c|c|c|c|c|}
\hline \multirow[t]{2}{*}{$\mathrm{R}_{H^{*}}^{B}$} & \multicolumn{3}{|c|}{ Observed } & \multicolumn{3}{|c|}{ Median expected } & \multirow[t]{2}{*}{ Assumption } \\
\hline & 0.5 & 1.0 & 2.0 & 0.5 & 1.0 & 2.0 & \\
\hline$\Gamma_{H} / \Gamma_{H}^{\mathrm{SM}}$ & 4.5 & 5.5 & 7.5 & 6.5 & 8.0 & 11.2 & $\kappa_{i, \text { on-shell }}=\kappa_{i, \text { off-shell }}$ \\
\hline$R_{g g}=\kappa_{g, \text { off-shell }}^{2} / \kappa_{g, \text { on-shell }}^{2}$ & 4.7 & 6.0 & 8.6 & 7.1 & 9.0 & 13.4 & $\kappa_{V, \text { on-shell }}=\kappa_{V, \text { off-shell }}, \Gamma_{H} / \Gamma_{H}^{\mathrm{SM}}=1$ \\
\hline
\end{tabular}


case the range is determined by varying the unknown $g g \rightarrow$ $Z Z$ and $g g \rightarrow W W$ background $\mathrm{K}$-factor from higher-order QCD corrections between half and twice the value of the known signal K-factor.

Assuming the relevant Higgs boson couplings are independent of the energy scale of the Higgs boson production, a combination with the on-shell measurements of $Z Z$ and $W W$ in the same dataset yields an observed (expected) $95 \%$ CL upper limit on $\Gamma_{H} / \Gamma_{H}^{S M}$ in the range 4.5-7.5 (6.511.2) under the same variations of the background $\mathrm{K}$-factor. Assuming the value of $\mathrm{R}_{H^{*}}^{B}=1$ and under the assumptions reported above, this translates into an observed (expected) $95 \%$ CL upper limit on the Higgs boson total width of 22.7 (33.0) $\mathrm{MeV}$.

Assuming that the total width of the Higgs boson is as expected in the SM, the same combination can be interpreted as a limit on the ratio of the off-shell to the on-shell couplings to gluons $R_{g g}=\kappa_{g \text {,off-shell }}^{2} / \kappa_{g, \text { on-shell }}^{2}$. An observed (expected) $95 \%$ CL upper limit on $R_{g g}$ in the range 4.7 8.6 (7.1-13.4) under the same variations of the background $\mathrm{K}$-factor is found.

Acknowledgments We are very thankful to M. Bonvini, J. Campbell, S. Forte, F. Krauss, K. Melnikov, G. Passarino, and M. Spannowsky for their essential input in the estimation of uncertainties in the theoretical predictions for the signal and background processes and their interference. We thank CERN for the very successful operation of the LHC, as well as the support staff from our institutions without whom ATLAS could not be operated efficiently. We acknowledge the support of ANPCyT, Argentina; YerPhI, Armenia; ARC, Australia; BMWFW and FWF, Austria; ANAS, Azerbaijan; SSTC, Belarus; CNPq and FAPESP, Brazil; NSERC, NRC and CFI, Canada; CERN; CONICYT, Chile; CAS, MOST and NSFC, China; COLCIENCIAS, Colombia; MSMT CR, MPO CR and VSC CR, Czech Republic; DNRF, DNSRC and Lundbeck Foundation, Denmark; EPLANET, ERC and NSRF, European Union; IN2P3-CNRS, CEA-DSM/IRFU, France; GNSF, Georgia; BMBF, DFG, HGF, MPG and AvH Foundation, Germany; GSRT and NSRF, Greece; RGC, Hong Kong SAR, China; ISF, MINERVA, GIF, I-CORE and Benoziyo Center, Israel; INFN, Italy; MEXT and JSPS, Japan; CNRST, Morocco; FOM and NWO, Netherlands; BRF and RCN, Norway; MNiSW and NCN, Poland; GRICES and FCT, Portugal; MNE/IFA, Romania; MES of Russia and NRC KI, Russian Federation; JINR; MSTD, Serbia; MSSR, Slovakia; ARRS and MIZŠ, Slovenia; DST/NRF, South Africa; MINECO, Spain; SRC and Wallenberg Foundation, Sweden; SER, SNSF and Cantons of Bern and Geneva, Switzerland; NSC, Taiwan; TAEK, Turkey; STFC, the Royal Society and Leverhulme Trust, United Kingdom; DOE and NSF, United States of America. The crucial computing support from all WLCG partners is acknowledged gratefully, in particular from CERN and the ATLAS Tier1 facilities at TRIUMF (Canada), NDGF (Denmark, Norway, Sweden), CC-IN2P3 (France), KIT/GridKA (Germany), INFN-CNAF (Italy), NL-T1 (Netherlands), PIC (Spain), ASGC (Taiwan), RAL (UK) and BNL (USA) and in the Tier-2 facilities worldwide.

Open Access This article is distributed under the terms of the Creative Commons Attribution 4.0 International License (http://creativecomm ons.org/licenses/by/4.0/), which permits unrestricted use, distribution, and reproduction in any medium, provided you give appropriate credit to the original author(s) and the source, provide a link to the Creative Commons license, and indicate if changes were made.

Funded by SCOAP ${ }^{3}$.
Appendix A: Monte Carlo and PDF scaling to arbitrary

\section{$\mu_{\text {off-shell }}$}

The known dependence of the off-shell Higgs boson signal process, the background process and the interference term on the off-shell signal strength $\mu_{\text {off-shell }}$ can be used to construct MC samples for arbitrary values of $\mu_{\text {off-shell }}$ from three basic samples generated at different fixed values of $\mu_{\text {off-shell }}$.

\section{A.1: Dependence of the $g g \rightarrow\left(H^{*} \rightarrow\right) V V$ off-shell cross-sections on the signal strength}

An event sample $\sigma_{g g \rightarrow\left(H^{*} \rightarrow\right) V V}\left(\mu_{\text {off-shell }}\right)$ for the $g g \rightarrow$ $\left(H^{*} \rightarrow\right) V V$ process with an arbitrary value of the offshell Higgs boson signal strength $\mu_{\text {off-shell }}$ can be constructed from the MC sample for the SM Higgs boson signal $g g \rightarrow H^{*} \rightarrow V V\left(\sigma_{g g \rightarrow H^{*} \rightarrow V V}^{\mathrm{SM}}\right)$, the $g g \rightarrow V V$ continuum background MC sample $\left(\sigma_{g g \rightarrow V V}\right.$, cont $)$ and a full SM Higgs boson signal plus background $g g \rightarrow\left(H^{*} \rightarrow\right) V V$ MC sample $\left(\sigma_{g g \rightarrow\left(H^{*} \rightarrow\right) V V}^{\mathrm{SM}}\right)$ using the following weighting function:

$$
\begin{aligned}
& \sigma_{g g \rightarrow\left(H^{*} \rightarrow\right) V V}\left(\mu_{\text {off-shell }}, m_{V V}\right) \\
& =\mathrm{K}^{H^{*}}\left(m_{V V}\right) \cdot \mu_{\text {off-shell }} \cdot \sigma_{g g \rightarrow H^{*} \rightarrow V V}^{\mathrm{SM}}\left(m_{V V}\right) \\
& +\sqrt{\mathrm{K}_{g g}^{H^{*}}\left(m_{V V}\right) \cdot \mathrm{K}^{\mathrm{B}}\left(m_{V V}\right) \cdot \mu_{\text {off-shell }}} \\
& \quad \cdot \sigma_{g g \rightarrow V V, \text { Interference }}^{\mathrm{SM}}\left(m_{V V}\right) \\
& +\mathrm{K}^{\mathrm{B}}\left(m_{V V}\right) \cdot \sigma_{g g \rightarrow V V, \text { cont }}\left(m_{V V}\right), \\
& \sigma_{g g \rightarrow V V, \text { Interference }}\left(m_{V V}\right) \\
& =\sigma_{g g \rightarrow\left(H^{*} \rightarrow\right) V V}^{\mathrm{SM}}\left(m_{V V}\right)-\sigma_{g g \rightarrow H^{*} \rightarrow V V}^{\mathrm{SM}}\left(m_{V V}\right) \\
& -\sigma_{g g \rightarrow V V, \text { cont }}\left(m_{V V}\right),
\end{aligned}
$$

where the K-factors are calculated inclusively without any selections.

As a direct simulation of an interference $\mathrm{MC}$ sample is not possible, Eq. (13) and $\mathrm{R}_{H^{*}}^{B}$ are used to obtain:

$$
\begin{aligned}
\sigma_{g g} \rightarrow\left(H^{*} \rightarrow\right) V V & \left(\mu_{\text {off-shell }}, m_{V V}\right) \\
= & \left(\mathrm{K}^{H^{*}}\left(m_{V V}\right) \cdot \mu_{\text {off-shell }}-\mathrm{K}_{g g}^{H^{*}}\left(m_{V V}\right)\right. \\
& \left.\cdot \sqrt{\mathbf{R}_{H^{*}}^{B} \cdot \mu_{\text {off-shell }}}\right) \cdot \sigma_{g g \rightarrow H^{*} \rightarrow V V}^{\mathrm{SM}}\left(m_{V V}\right) \\
+ & \mathrm{K}_{g g}^{H^{*}}\left(m_{V V}\right) \cdot \sqrt{\mathbf{R}_{H^{*}}^{B} \cdot \mu_{\text {off-shell }}} \\
& \cdot \sigma_{g g \rightarrow\left(H^{*} \rightarrow\right) V V}^{\mathrm{SM}}\left(m_{V V}\right) \\
+ & \mathrm{K}_{g g}^{H^{*}}\left(m_{V V}\right) \cdot\left(\mathrm{R}_{H^{*}}^{B}-\sqrt{\mathbf{R}_{H^{*}}^{B} \cdot \mu_{\text {off-shell }}}\right) . \\
& \cdot \sigma_{g g \rightarrow V V, \text { cont }}\left(m_{V V}\right) .
\end{aligned}
$$


A.2: Dependence of the $V V+2 j$ off-shell signal and background interference on the signal strength

An MC event sample for the EW $p p \rightarrow\left(H^{*}+2 j \rightarrow\right) V V$ $+2 j$ process with an arbitrary value of the off-shell Higgs boson signal strength $\mu_{\text {off-shell }}$ can be constructed from a pure $p p \rightarrow V V+2 j$ continuum background MC sample, a full SM Higgs boson signal plus background $p p \rightarrow$ $\left(H^{*}+2 j \rightarrow\right) V V+2 j$ MC sample and a third Higgs boson signal plus background $p p \rightarrow\left(H^{*}+2 j \rightarrow\right)$ $V V+2 j$ MC sample with $\mu_{\text {off-shell }}=\kappa_{V}^{4}=\Gamma_{H} / \Gamma_{H}^{\mathrm{SM}}=10$. Using $\Gamma_{H} / \Gamma_{H}^{\mathrm{SM}}=10$ for the last sample ensures that the onshell $\mathrm{VH}$ events are generated with SM-like signal strength.

The following weighting function is used:

$$
\begin{aligned}
& \sigma_{p p \rightarrow\left(H^{*}+2 j \rightarrow\right) V V+2 j}\left(\mu_{\mathrm{off}-\text { shell }}\right) \\
& \quad=\mu_{\mathrm{off}-\text { shell }} \cdot \sigma_{p p \rightarrow H^{*}+2 j \rightarrow V V+2 j}^{\mathrm{SM}} \\
& +\sqrt{\mu_{\mathrm{off}-\text { shell }}} \cdot \sigma_{p p \rightarrow V V+2 j, \text { Interference }}+\sigma_{p p \rightarrow V V+2 j, \text { cont }},
\end{aligned}
$$

where the signal and interference samples are implicitly defined through the SM $p p \rightarrow\left(H^{*}+2 j \rightarrow\right) V V+2 j$ MC sample

$$
\begin{aligned}
\sigma_{p p \rightarrow\left(H^{*}+2 j \rightarrow\right) V V+2 j}^{\mathrm{SM}} & =\sigma_{p p \rightarrow H^{*}+2 j \rightarrow V V+2 j}^{\mathrm{SM}} \\
& +\sigma_{p p \rightarrow V V+2 j, \text { Interference }} \\
& +\sigma_{p p \rightarrow V V+2 j, \mathrm{cont}}
\end{aligned}
$$

and a $\mu_{\text {off-shell }}=10 \mathrm{MC}$ sample:

$$
\begin{aligned}
\sigma_{p p \rightarrow\left(H^{*}+2 j \rightarrow\right) V V+2 j}^{\kappa_{V}^{4}=10} & =10 \cdot \sigma_{p p \rightarrow H^{*}+2 j \rightarrow V V+2 j}^{\mathrm{SM}} \\
& +\sqrt{10} \cdot \sigma_{p p \rightarrow V V+2 j, \text { Interference }} \\
& +\sigma_{p p \rightarrow V V+2 j, \text { cont }} .
\end{aligned}
$$

Solving for the generated MC samples yields:

$$
\begin{aligned}
& \sigma_{p p \rightarrow\left(H^{*}+2 j \rightarrow\right) V V+2 j}\left(\mu_{\text {off-shell }}\right) \\
& =\frac{\mu_{\text {off-shell }}-\sqrt{\mu_{\text {off-shell }}}}{10-\sqrt{10}} \sigma_{p p \rightarrow\left(H^{*}+2 j \rightarrow\right) V V+2 j}^{\kappa_{V}^{4}=10} \\
& +\frac{10 \sqrt{\mu_{\text {off-shell }}}-\sqrt{10} \mu_{\text {off-shell }}}{10-\sqrt{10}} \sigma_{p p \rightarrow\left(H^{*}+2 j \rightarrow\right) V V+2 j}^{\mathrm{SM}} \\
& +\frac{\left(\sqrt{\mu_{\text {off-shell }}}-1\right) \cdot\left(\sqrt{\mu_{\text {off-shell }}}-\sqrt{10}\right)}{\sqrt{10}} \sigma_{p p \rightarrow V V+2 j, \text { cont }} .
\end{aligned}
$$

\section{References}

1. ATLAS Collaboration, Observation of a new particle in the search for the Standard Model Higgs boson with the ATLAS detector at the LHC. Phys. Lett. B 716, 1 (2012). arXiv:1207.7214 [hep-ex]

2. CMS Collaboration, Observation of a new boson at a mass of 125 $\mathrm{GeV}$ with the CMS experiment at the LHC. Phys. Lett. B 716, 30 (2012). arXiv:1207.7235 [hep-ex]
3. ATLAS Collaboration, Measurements of Higgs boson production and couplings in diboson final states with the ATLAS detector at the LHC. Phys. Lett. B 726, 88 (2013). arXiv:1307.1427 [hep-ex]

4. CMS Collaboration, Precise determination of the mass of the Higgs boson and tests of compatibility of its couplings with the standard model predictions using proton collisions at 7 and $8 \mathrm{TeV}$. Eur. Phys. J. C 75(5), 212 (2015). arXiv:1412.8662 [hep-ex]

5. ATLAS Collaboration, Evidence for the spin- 0 nature of the Higgs boson using ATLAS data. Phys. Lett. B 726, 120 (2013). arXiv:1307.1432 [hep-ex]

6. CMS Collaboration, Constraints on the spin-parity and anomalous HVV couplings of the Higgs boson in proton collisions at 7 and 8 TeV. arXiv:1411.3441 [hep-ex]

7. N. Kauer, G. Passarino, Inadequacy of zero-width approximation for a light Higgs boson signal. JHEP 08, 116 (2012). arXiv:1206.4803 [hep-ph]

8. F. Caola, K. Melnikov, Constraining the Higgs boson width with ZZ production at the LHC. Phys. Rev. D 88, 054024 (2013). arXiv:1307.4935 [hep-ph]

9. J.M. Campbell, R.K. Ellis, C. Williams, Bounding the Higgs width at the LHC using full analytic results for $g g \rightarrow e^{-} e^{+} \mu^{-} \mu^{+}$. JHEP 04, 060 (2014). arXiv:1311.3589 [hep-ph]

10. J.M. Campbell, R.K. Ellis, C. Williams, Bounding the Higgs width at the LHC: complementary results from $H \rightarrow W W$. Phys. Rev. D 89, 053011 (2014). arXiv:1312.1628 [hep-ph]

11. ATLAS Collaboration, Measurement of the Higgs boson mass from the $H \rightarrow \gamma \gamma$ collision data. Phys. Rev. D 90, 052004 (2014). arXiv: 1406.3827 [hep-ex]

12. C. Englert, M. Spannowsky, Limitations and opportunities of offshell coupling measurements. Phys. Rev. D 90, 053003 (2014). arXiv: 1405.0285 [hep-ph]

13. G. Cacciapaglia, A. Deandrea, G.D. La Rochelle, J.-B. Flament, Higgs couplings: disentangling new physics with off-shell measurements. Phys. Rev. Lett. 113, 201802 (2014). arXiv:1406.1757 [hep-ph]

14. A. Azatov, C. Grojean, A. Paul, E. Salvioni, Taming the off-shell Higgs boson. JETP 147(3) (2015). arXiv:1406.6338 [hep-ph]

15. M. Ghezzi, G. Passarino, S. Uccirati, Bounding the Higgs width using effective field theory. PoS LL2014, 072 (2014). arXiv:1405.1925 [hep-ph]

16. M. Buschmann, D. Goncalves, S. Kuttimalai, M. Schonherr, F. Krauss et al., Mass effects in the Higgs-gluon coupling: boosted vs off-shell production. JHEP 2, 38 (2015). doi:10.1007/ JHEP02(2015)038. arXiv:1410.5806 [hep-ph]

17. J.S. Gainer, J. Lykken, K.T. Matchev, S. Mrenna, M. Park, Beyond geolocating: constraining higher dimensional operators in $H \rightarrow 4 \ell$ with off-shell production and more. Phys. Rev. D 91(3), 035011 (2015). arXiv:1403.4951 [hep-ph]

18. C. Englert, Y. Soreq, M. Spannowsky, Off-shell Higgs coupling measurements in BSM scenarios. JHEP 5, 145 (2015). doi: 10.1007/ JHEP05(2015)145. arXiv:1410.5440 [hep-ph]

19. CMS Collaboration, Constraints on the Higgs boson width from off-shell production and decay to $Z$-boson pairs. Phys. Lett. B 736, 64 (2014). arXiv: 1405.3455 [hep-ex]

20. ATLAS Collaboration, Search for invisible decays of a Higgs boson produced in association with a Z boson in ATLAS. Phys. Rev. Lett. 112, 201802 (2014). arXiv:1402.3244 [hep-ex]

21. CMS collaboration, Search for invisible decays of Higgs bosons in the vector boson fusion and associated $\mathrm{ZH}$ production modes. Eur. Phys. J. C 74, 2980 (2014). arXiv:1404.1344 [hep-ex]

22. ATLAS Collaboration, The ATLAS experiment at the CERN Large Hadron Collider. JINST 3, S08003 (2008)

23. LHC Higgs Cross Section Working Group, S. Heinemeyer, C. Mariotti, G. Passarino, R. Tanaka (eds.), Handbook of LHC Higgs Cross Sections: 3. Higgs Properties. arXiv:1307.1347 [hep-ph] 
24. H.E. Logan, Hiding a Higgs width enhancement from off-shell gg $\left(\rightarrow h^{*}\right) \rightarrow$ ZZ measurements. arXiv:1412.7577 [hep-ph]

25. G. Passarino, Higgs CAT. Eur. Phys. J. C 74, 2866 (2014). arXiv:1312.2397 [hep-ph]

26. S. Agostinelli et al., Geant4, a simulation toolkit. Nucl. Instrum. Methods A 506, 250 (2003)

27. ATLAS Collaboration, The ATLAS simulation infrastructure. Eur. Phys. J. C 70, 823 (2010). arXiv:1005.4568 [hep-ph]

28. N. Kauer, Interference effects for $\mathrm{H} \rightarrow \mathrm{WW} / \mathrm{ZZ} \rightarrow \ell \bar{v}_{\ell} \bar{\ell} v_{\ell}$ searches in gluon fusion at the LHC. JHEP 12, 082 (2013). arXiv:1310.7011 [hep-ph]

29. T. Sjostrand, S. Mrenna, P.Z. Skands, A. Brief, Introduction to PYTHIA 8.1. Comput. Phys. Commun. 178, 852-867 (2008). arXiv:0710.3820 [hep-ph]

30. F. Cascioli et al., Precise Higgs-background predictions: merging NLO QCD and squared quark-loop corrections to four-lepton + 0,1 jet production. JHEP 01, 046 (2014). arXiv:1309.0500 [hep-ph]

31. T. Gleisberg et al., Event generation with SHERPA 1.1. JHEP 02, 007 (2009). arXiv:0811.4622 [hep-ph]

32. F. Cascioli, P. Maierhöfer, S. Pozzorini, Scattering amplitudes with open loops. Phys. Rev. Lett. 108, 111601 (2012). arXiv:1111.5206 [hep-ph]

33. A. Denner, S. Dittmaier, L. Hofer, COLLIER - a fortran-library for one-loop integrals. PoS LL2014, 071 (2014). arXiv:1407.0087 [hep-ph]

34. J. Gao et al., The CT10 NNLO global analysis of QCD. Phys. Rev. D 89, 033009 (2014). arXiv:1302.6246 [hep-ph]

35. A. Martin, W. Stirling, R. Thorne, G. Watt, Parton distributions for the LHC. Eur. Phys. J. C 63, 189-285 (2009). arXiv:0901.0002 [hep-ph]

36. M. Bonvini, F. Caola, S. Forte, K. Melnikov, G. Ridolfi, Signalbackground interference effects for $g g \rightarrow H \rightarrow W^{+} W^{-}$beyond leading order. Phys. Rev. D 88, 034032 (2013). arXiv:1304.3053 [hep-ph]

37. J. Alwall et al., The automated computation of tree-level and nextto-leading order differential cross sections, and their matching to parton shower simulations. JHEP 07, 079 (2014). arXiv:1405.0301 [hep-ph]. (optimised by the authors for the production of VBF $\left.\left(H^{*} \rightarrow\right) V V\right)$

38. A. Ballestrero, A. Belhouari, G. Bevilacqua, V. Kashkan, E. Maina, PHANTOM: a Monte Carlo event generator for six parton final states at high energy colliders. Comput. Phys. Commun. 180, 401417 (2009). arXiv:0801.3359 [hep-ph]. (extended by the authors to include the production of the $\left.\mathrm{VBF} H^{*}\right) \rightarrow V V$ signal process

39. LHC Higgs Cross Section Working Group, S. Dittmaier, C. Mariotti, G. Passarino, R. Tanaka (eds.), Handbook of LHC Higgs Cross Sections: 2. Differential Distributions, CERN-2012-002 (CERN, Geneva, 2012). arXiv:1201.3084 [hep-ph]

40. J. Pumplin et al., New generation of parton distributions with uncertainties from global QCD analysis. JHEP 07, 012 (2002). arXiv:hep-ph/0201195

41. T. Sjostrand et al., PYTHIA 6.4 physics and manual. JHEP $\mathbf{0 5}, 026$ (2006)

42. T. Melia, P. Nason, R. Röntsch, G. Zanderighi, $\mathrm{W}^{+} \mathrm{W}^{-}, \mathrm{WZ}$ and ZZ production in the POWHEG BOX. JHEP 11, 078 (2011). arXiv:1107.5051 [hep-ph]

43. F. Cascioli et al., ZZ production at hadron colliders in NNLO QCD. Phys. Lett. B 735, 311 (2014). arXiv:1405.2219 [hep-ph]. (extended by the authors to provide NNLO/NLO K-factors as a function of $m_{Z Z}$ )
44. T. Gehrmann et al., $W^{+} W^{-}$production at hadron colliders in NNLO QCD. Phys. Rev. Lett. 113, 212001 (2014). arXiv:1408.5243 [hep-ph]. (extended by the authors to provide NNLO/NLO K-factors as a function of $m_{W W}$ )

45. A. Bierweiler, T. Kasprzik, J.H. Kühn, Vector-boson pair production at the LHC to $\mathcal{O}\left(\alpha^{3}\right)$ accuracy. JHEP 12, 071 (2013). arXiv:1305.5402 [hep-ph]

46. J. Baglio, L.D. Ninh, M.M. Weber, Massive gauge boson pair production at the LHC: a next-to-leading order story. Phys. Rev. D 88, 113005 (2013). arXiv: 1307.4331

47. S. Gieseke, T. Kasprzik, J.H. Kühn, Vector-boson pair production and electroweak corrections in HERWIG++. Eur. Phys. J. C 74, $2988(2014)$

48. S. Alioli, S.-O. Moch, P. Uwer, Hadronic top-quark pair-production with one jet and parton showering. JHEP 1201, 137 (2012). arXiv: 1110.5251 [hep-ph]

49. S. Alioli, P. Nason, C. Oleari, E. Re, NLO single-top production matched with shower in POWHEG: s- and t-channel contributions. JHEP 0909, 111 (2009). arXiv:0907.4076 [hep-ph]

50. B.P. Kersevan, E. Richter-Was, The Monte Carlo event generator AcerMC version 2.0 with interfaces to PYTHIA 6.2 and HERWIG 6.5. arXiv:hep-ph/0405247

51. M. Czakon, A. Mitov, Top++: a program for the calculation of the top-pair cross-section at hadron colliders. Comput. Phys. Commun. 185(11), 2930-2938 (2014). http://www.sciencedirect.com/ science/article/pii/S0010465514002264

52. N. Kidonakis, Next-to-next-to-leading logarithm resummation for $s$-channel single top quark production. Phys. Rev. D 81, 054028 (2010). doi:10.1103/PhysRevD.81.054028

53. N. Kidonakis, Next-to-next-to-leading-order collinear and soft gluon corrections for $t$-channel single top quark production. Phys. Rev. D 83, 091503 (2011). doi:10.1103/PhysRevD.83.091503

54. N. Kidonakis, Two-loop soft anomalous dimensions for single top quark associated production with a $W^{-}$. Phys. Rev. D 82, 054018 (2010). doi:10.1103/PhysRevD.82.054018

55. ATLAS Collaboration, Measurements of Higgs boson production and couplings in the four-lepton channel in pp collisions at centerof-mass energies of 7 and $8 \mathrm{TeV}$ with the ATLAS detector. Phys. Rev. D 91, 012006 (2015). arXiv:1408.5191 [hep-ex]

56. ATLAS Collaboration, Calibration of $b$-tagging using dileptonic top pair events in a combinatorial likelihood approach with the ATLAS experiment, ATLAS-CONF-2014-004 (2014). https://cds. cern.ch/record/1664335

57. ATLAS Collaboration, Calibration of the performance of $b$ and light-flavour jets in the 2012 ATLAS data, ATLAS-CONF-2014046 (2014). https://cds.cern.ch/record/1741020

58. ATLAS Collaboration, Observation and measurement of Higgs boson decays to $W W^{*}$ with the ATLAS detector. arXiv:1412.2641 [hep-ex]

59. G. Corcella et al., HERWIG 6: an event generator for hadron emission reactions with interfering gluons (including supersymmetric processes). JHEP 01, 010 (2001)

60. H.-L. Lai et al., New parton distributions for collider physics. Phys. Rev. D 82, 074024 (2010)

61. R.D. Ball et al., Impact of heavy quark masses on parton distributions and LHC phenomenology. Nucl. Phys. B 849, 296 (2011). arXiv:1101.1300 [hep-ph] 
62. M. Botje et al., The PDF4LHC working group interim recommendations. arXiv:1101.0538 [hep-ph]

63. ATLAS Collaboration, Improved luminosity determination in $p p=7 \mathrm{TeV}$ using the ATLAS detector at the LHC. Eur. Phys. J. C 73, 2518 (2013). arXiv:1302.4393 [hep-ex]

64. G. Cowan, K. Cranmer, E. Gross, O. Vitells, Asymptotic formulae for likelihood-based tests of new physics. Eur. Phys. J. C 71, 1554 (2011). arXiv:1007.1727 [physics.data-an]
65. A.L. Read, Presentation of search results: the $\mathrm{CL}_{s}$ technique. J. Phys. G28, 2693 (2002)

66. ATLAS Collaboration, Combined search for the Standard Model Higgs boson in $p p=7 \mathrm{TeV}$ with the ATLAS detector. Phys. Rev. D 86, 032003 (2012). arXiv:1207.0319 [hep-ex]

\section{ATLAS Collaboration}

G. Aad ${ }^{85}$, B. Abbott ${ }^{113}$, J. Abdallah ${ }^{152}$, O. Abdinov ${ }^{11}$, R. Aben ${ }^{107}$, M. Abolins ${ }^{90}$, O. S. AbouZeid ${ }^{159}$, H. Abramowicz ${ }^{154}$, H. Abreu ${ }^{153}$, R. Abreu ${ }^{30}$, Y. Abulaiti ${ }^{147 a, 147 b}$, B. S. Acharya ${ }^{165 a, 165 b, a}$, L. Adamczyk ${ }^{38 a}$, D. L. Adams ${ }^{25}$, J. Adelman ${ }^{108}$, S. Adomeit ${ }^{100}$, T. Adye ${ }^{131}$, A. A. Affolder ${ }^{74}$, T. Agatonovic-Jovin ${ }^{13}$, J. A. Aguilar-Saavedra ${ }^{126 a, 126 f}$, M. Agustoni ${ }^{17}$, S. P. Ahlen ${ }^{22}$, F. Ahmadov ${ }^{65, b}$, G. Aielli ${ }^{134 a, 134 b}$, H. Akerstedt ${ }^{147 a, 147 b}$, T. P. A. Åkesson ${ }^{81}$, G. Akimoto ${ }^{156}$, A. V. Akimov ${ }^{96}$,

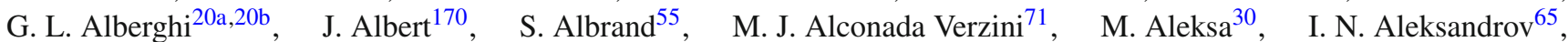
C. Alexa ${ }^{26 a}$, G. Alexander ${ }^{154}$, T. Alexopoulos ${ }^{10}$, M. Alhroob ${ }^{113}$, G. Alimonti ${ }^{91 a}$, L. Alio ${ }^{85}$, J. Alison ${ }^{31}$, S. P. Alkire ${ }^{35}$, B. M. M. Allbrooke ${ }^{18}$, P. P. Allport ${ }^{74}$, A. Aloisio ${ }^{104 a, 104 b}$, A. Alonso ${ }^{36}$, F. Alonso ${ }^{71}$, C. Alpigiani ${ }^{76}$, A. Altheimer ${ }^{35}$, B. Alvarez Gonzalez ${ }^{90}$, D. Álvarez Piqueras ${ }^{168}$, M. G. Alviggi ${ }^{104 a, 104 b}$, K. Amako ${ }^{66}$, Y. Amaral Coutinho ${ }^{24 a}$, C. Amelung ${ }^{23}$, D. Amidei ${ }^{89}$, S. P. Amor Dos Santos ${ }^{126 a, 126 c}$, A. Amorim ${ }^{126 a, 126 b}$, S. Amoroso 48 , N. Amram ${ }^{154}$, G. Amundsen ${ }^{23}$,

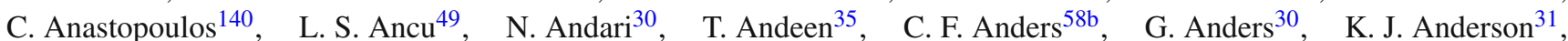
A. Andreazza ${ }^{91 a, 91 b}$, V. Andrei ${ }^{58 a}$, S. Angelidakis ${ }^{9}$, I. Angelozzi ${ }^{107}$, P. Anger ${ }^{44}$, A. Angerami ${ }^{35}$, F. Anghinolfi ${ }^{30}$, A. V. Anisenkov ${ }^{109, \mathrm{c}}, \quad$ N. Anjos ${ }^{12}$, A. Annovi ${ }^{124 a, 124 b}$, M. Antonelli ${ }^{47}, \quad$ A. Antonov ${ }^{98}$, J. Antos ${ }^{145 b}$, F. Anulli ${ }^{133 a}$, M. Aoki ${ }^{66}$, L. Aperio Bella ${ }^{18}$, G. Arabidze 90 , Y. Arai ${ }^{66}$, J. P. Araque ${ }^{126 a}$, A. T. H. Arce ${ }^{45}$, F. A. Arduh ${ }^{71}$, J-F. Arguin ${ }^{95}$, S. Argyropoulos ${ }^{42}$, M. Arik ${ }^{19 a}$, A. J. Armbruster ${ }^{30}$, O. Arnaez ${ }^{30}$, V. Arnal ${ }^{82}$, H. Arnold ${ }^{48}$, M. Arratia ${ }^{28}$, O. Arslan ${ }^{21}$, A. Artamonov ${ }^{97}$, G. Artoni ${ }^{23}$, S. Asai ${ }^{156}$, N. Asbah ${ }^{42}$, A. Ashkenazi ${ }^{154}$, B. Åsman ${ }^{147 a, 147 b}$, L. Asquith ${ }^{150}$, K. Assamagan ${ }^{25}$,

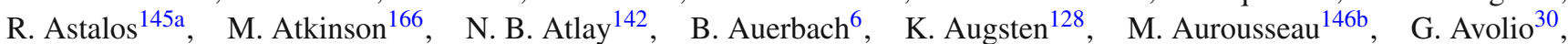
B. Axen ${ }^{15}$, M. K. Ayoub ${ }^{117}$, G. Azuelos ${ }^{95, d}$, M. A. Baak ${ }^{30}$, A. E. Baas ${ }^{58 a}, \quad$ C. Bacci135a,135b , H. Bachacou ${ }^{137}$, K. Bachas ${ }^{155}$, M. Backes ${ }^{30}$, M. Backhaus ${ }^{30}$, E. Badescu ${ }^{26 a}$, P. Bagiacchi ${ }^{133 a, 133 b}$, P. Bagnaia133a,133b, Y. Bai ${ }^{33 a}$, T. Bain ${ }^{35}$ ， J. T. Baines ${ }^{131}$ ， O. K. Baker ${ }^{177}$ ， P. Balek ${ }^{129}$ ， T. Balestri ${ }^{149}$ ， F. Balli ${ }^{84}$ ， E. Banas ${ }^{39}$, Sw. Banerjee ${ }^{174}$, A. A. E. Bannoura ${ }^{176}$, H. S. Bansil ${ }^{18}$, L. Barak ${ }^{30}$, S. P. Baranov ${ }^{96}$, E. L. Barberio ${ }^{88}$, D. Barberis ${ }^{50 a, 50 b}$, M. Barbero ${ }^{85}$, T. Barillari ${ }^{101}$, M. Barisonzi ${ }^{165 a, 165 b}$, T. Barklow ${ }^{144}$, N. Barlow ${ }^{28}$, S. L. Barnes ${ }^{84}$, B. M. Barnett ${ }^{131}$, R. M. Barnett ${ }^{15}$, Z. Barnovska ${ }^{5}$, A. Baroncelli135a， G. Barone ${ }^{49}$, A. J. Barr ${ }^{120}$, F. Barreiro ${ }^{82}$, J. Barreiro Guimarães da Costa ${ }^{57}$, R. Bartoldus ${ }^{144}$, A. E. Barton ${ }^{72}$, P. Bartos ${ }^{145 a}$, A. Bassalat ${ }^{117}$, A. Basye ${ }^{166}$, R. L. Bates ${ }^{53}$, S. J. Batista ${ }^{159}$, J. R. Batley ${ }^{28}$,

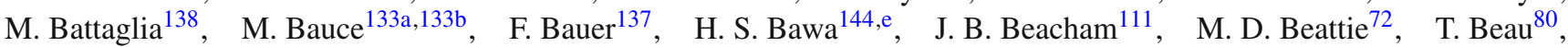
P. H. Beauchemin ${ }^{162}$ ，R. Beccherle ${ }^{124 a, 124 b}$ ，P. Bechtle ${ }^{21}$, H. P. Beck ${ }^{17, f}$, K. Becker ${ }^{120}$, M. Becker ${ }^{83}$, S. Becker ${ }^{100}$, M. Beckingham ${ }^{171}$ ，C. Becot ${ }^{117}$ ，A. J. Beddall ${ }^{19 c}$ ，A. Beddall ${ }^{19 c}$ ，V. A. Bednyakov ${ }^{65}$ ，C. P. Bee ${ }^{149}$ ， L. J. Beemster ${ }^{107}$, T. A. Beermann ${ }^{176}$, M. Begel ${ }^{25}$, J. K. Behr ${ }^{120}$, C. Belanger-Champagne ${ }^{87}$, W. H. Bell' ${ }^{49}$, G. Bella ${ }^{154}$, L. Bellagamba ${ }^{20 a}$, A. Bellerive ${ }^{29}$, M. Bellomo ${ }^{86}$, K. Belotskiy ${ }^{98}$, O. Beltramello ${ }^{30}$, O. Benary ${ }^{154}$, D. Benchekroun ${ }^{136 a}$, M. Bender ${ }^{100}$, K. Bendtz ${ }^{147 a, 147 b}$, N. Benekos ${ }^{10}$, Y. Benhammou ${ }^{154}$, E. Benhar Noccioli ${ }^{49}$, J. A. Benitez Garcia ${ }^{160 b}$, D. P. Benjamin ${ }^{45}$, J. R. Bensinger ${ }^{23}$, S. Bentvelsen ${ }^{107}$, L. Beresford ${ }^{120}$, M. Beretta ${ }^{47}$, D. Berge ${ }^{107}$, E. Bergeaas Kuutmann ${ }^{167}$, N. Berger ${ }^{5}$, F. Berghaus ${ }^{170}$, J. Beringer ${ }^{15}$, C. Bernard ${ }^{22}$, N. R. Bernard ${ }^{86}$, C. Bernius ${ }^{110}$, F. U. Bernlochner ${ }^{21}, \quad$ T. Berry ${ }^{77}$, P. Berta ${ }^{129}$ ， C. Bertella ${ }^{83}$ ，G. Bertoli ${ }^{147 a, 147 b}$, F. Bertolucci124a,124b , C. Bertsche ${ }^{113}$ ，D. Bertsche ${ }^{113}$, M. I. Besana ${ }^{91 a}$, G. J. Besjes ${ }^{106}$, O. Bessidskaia Bylund ${ }^{147 a, 147 b}$, M. Bessner ${ }^{42}$, N. Besson ${ }^{137}$, C. Betancourt ${ }^{48}$, S. Bethke ${ }^{101}$, A. J. Bevan ${ }^{76}$, W. Bhimji ${ }^{46}$, R. M. Bianchi ${ }^{125}$, L. Bianchini ${ }^{23}, \quad$ M. Bianco ${ }^{30}, \quad$ O. Biebel ${ }^{100}, \quad$ S. P. Bieniek ${ }^{78}, \quad$ M. Biglietti ${ }^{135 a}$, J. Bilbao De Mendizabal ${ }^{49}$, H. Bilokon ${ }^{47}$, M. Bindi ${ }^{54}$, S. Binet ${ }^{117}$, A. Bingul ${ }^{19 c}$, C. Bini ${ }^{133 a, 133 b}$, C. W. Black ${ }^{151}$, $\begin{array}{ll}\text { J. E. Black } & 144\end{array}$ K. M. Black ${ }^{22}$, D. Blackburn ${ }^{139}$ ， R. E. Blair ${ }^{6}$, J.-B. Blanchard ${ }^{137}$ ， J. E. Blanco ${ }^{77}$, T. Blazek ${ }^{145 a}$, ${\text { I. } \text { Bloch }^{42} \text {, C. Blocker }}^{23}$, W. Blum ${ }^{83, *}$, U. Blumenschein ${ }^{54}$, G. J. Bobbink ${ }^{107}$, V. S. Bobrovnikov ${ }^{109, \text { c }, ~ S . ~ S . ~ B o c c h e t t a ~}{ }^{81}$, A. Bocci ${ }^{45}$ ， C. Bock ${ }^{100}$ ， M. Boehler ${ }^{48}$ ， J. A. Bogaerts ${ }^{30}, \quad$ A. G. Bogdanchikov ${ }^{109}, \quad$ C. Bohm ${ }^{147 a}$ ， V. Boisvert ${ }^{77}$, T. Bold ${ }^{38 a}$, V. Boldea ${ }^{26 a}$, A. S. Boldyrev ${ }^{99}$, M. Bomben ${ }^{80}$, M. Bona ${ }^{76}$, M. Boonekamp ${ }^{137}$, A. Borisov ${ }^{130}$, G. Borissov ${ }^{72}$, S. Borroni ${ }^{42}$ ， J. Bortfeldt ${ }^{100}$ ， V. Bortolotto ${ }^{60 a, 60 b, 60 c}$, K. Bos ${ }^{107}$ ， D. Boscherini ${ }^{20 a}$ ， M. Bosman ${ }^{12}$ ， J. Boudreau ${ }^{125}$, J. Bouffard ${ }^{2}$ ， E. V. Bouhova-Thacker ${ }^{72}$ ， D. Boumediene ${ }^{34}, \quad$ C. Bourdarios ${ }^{117}, \quad$ N. Bousson ${ }^{114}$, S. Boutouil ${ }^{136 d}$, A. Boveia ${ }^{30}$, J. Boyd ${ }^{30}$, I. R. Boyko ${ }^{65}$, I. Bozic ${ }^{13}$, J. Bracinik ${ }^{18}$, A. Brandt ${ }^{8}$, G. Brandt ${ }^{15}$, O. Brandt ${ }^{58 a}$, U. Bratzler ${ }^{157}$, 
B. Brau ${ }^{86}$ ，J. E. Brau ${ }^{116}$, H. M. Braun ${ }^{176, *}$, S. F. Brazzale ${ }^{165 a, 165 c}$ ，K. Brendlinger ${ }^{122}$ ，A. J. Brennan ${ }^{88}$ ， L. Brenner ${ }^{107}$, R. Brenner ${ }^{167}$, S. Bressler ${ }^{173}$, K. Bristow ${ }^{146 c}$, T. M. Bristow ${ }^{46}$, D. Britton ${ }^{53}$, D. Britzger ${ }^{42}$, F. M. Brochu ${ }^{28}$, I. Brock ${ }^{21}$, R. Brock ${ }^{90}$, J. Bronner ${ }^{101}$, G. Brooijmans ${ }^{35}$, T. Brooks ${ }^{77}$, W. K. Brooks ${ }^{32 b}$, J. Brosamer ${ }^{15}$, E. Brost ${ }^{116}$, J. Brown ${ }^{55}$, P. A. Bruckman de Renstrom ${ }^{39}$, D. Bruncko ${ }^{145 b}$, R. Bruneliere ${ }^{48}$, A. Bruni ${ }^{20 a}$, G. Bruni $^{20 a}$, M. Bruschi ${ }^{20 a}$, L. Bryngemark $^{81}$, T. Buanes ${ }^{14}$, Q. Buat ${ }^{143}$, P. Buchholz ${ }^{142}$, A. G. Buckley ${ }^{53}$, S. I. Buda ${ }^{26 a}$, I. A. Budagov ${ }^{65}$, F. Buehrer ${ }^{48}$, L. Bugge ${ }^{119}$, M. K. Bugge ${ }^{119}$, O. Bulekov ${ }^{98}$, H. Burckhart ${ }^{30}, \quad$ S. Burdin ${ }^{74}$, B. Burghgrave ${ }^{108}$, S. Burke ${ }^{131}$, I. Burmeister ${ }^{43}$, E. Busato ${ }^{34}$, D. Büscher ${ }^{48}$, V. Büscher ${ }^{83}$, P. Bussey ${ }^{53}$, C. P. Buszello ${ }^{167}$, J. M. Butler22, A. I. Butt ${ }^{3}$, C. M. Buttar ${ }^{53}$, J. M. Butterworth ${ }^{78}$, P. Butti ${ }^{107}$, W. Buttinger ${ }^{25}$, A. Buzatu ${ }^{53}$, R. Buzykaev ${ }^{109, c}$, S. Cabrera Urbán ${ }^{168}$, D. Caforio ${ }^{128}$, O. Cakir $^{4 a}$, P. Calafiura ${ }^{15}$, A. Calandri ${ }^{137}$, G. Calderini ${ }^{80}$, P. Calfayan ${ }^{100}$, L. P. Caloba ${ }^{24 a}$, D. Calvet ${ }^{34}$, S. Calvet ${ }^{34}$,

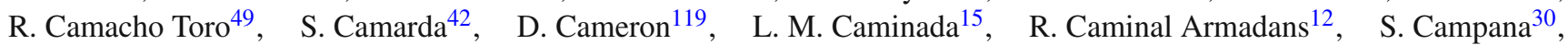
M. Campanelli ${ }^{78}$, A. Campoverde ${ }^{149}$, V. Canale ${ }^{104 a, 104 b}$, A. Canepa ${ }^{160 a}$, M. Cano Bret ${ }^{76}$, J. Cantero ${ }^{82}$, R. Cantrill ${ }^{126 a}$, T. Cao ${ }^{40}$, M. D. M. Capeans Garrido ${ }^{30}$, I. Caprini ${ }^{26 a}$, M. Caprini ${ }^{26 a}$, M. Capua ${ }^{37 a}$,37b, R. Caputo ${ }^{83}$, R. Cardarelli ${ }^{134 a}$, T. Carli $^{30}$, G. Carlino104a, L. Carminati ${ }^{11 a, 91 b}$, S. Caron ${ }^{106}$, E. Carquin ${ }^{32 a}$ ， G. D. Carrillo-Montoya ${ }^{8}$, J. R. Carter ${ }^{28}$,

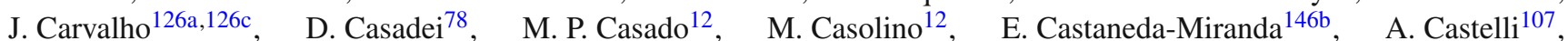
V. Castillo Gimenez ${ }^{168}$, N. F. Castro ${ }^{126 a, g}$, P. Catastini ${ }^{57}$, A. Catinaccio ${ }^{30}$, J. R. Catmore ${ }^{119}$, A. Cattai ${ }^{30}$, J. Caudron ${ }^{83}$, V. Cavaliere ${ }^{166}$, D. Cavalli ${ }^{91 a}$, M. Cavalli-Sforza ${ }^{12}$, V. Cavasinni ${ }^{124 a, 124 b}$, F. Ceradini ${ }^{135 a, 135 b}$, B. C. Cerio ${ }^{45}$, K. Cerny ${ }^{129}$, A. S. Cerqueira ${ }^{24 b}$, A. Cerri ${ }^{150}$, L. Cerrito ${ }^{76}$, F. Cerutti ${ }^{15}$, M. Cerv ${ }^{30}$, A. Cervelli ${ }^{17}$, S. A. Cetin ${ }^{19 b}$, A. Chafaq ${ }^{136 a}$, D. Chakraborty ${ }^{108}$, I. Chalupkova ${ }^{129}$, P. Chang ${ }^{166}$, B. Chapleau ${ }^{87}$, J. D. Chapman ${ }^{28}$, D. G. Charlton ${ }^{18}$, C. C. Chau ${ }^{159}$, C. A. Chavez Barajas ${ }^{150}$, S. Cheatham ${ }^{153}$, A. Chegwidden ${ }^{90}$, S. Chekanov ${ }^{6}$, S. V. Chekulaev ${ }^{160 a}$, G. A. Chelkov ${ }^{65, h}$, M. A. Chelstowska ${ }^{89}$, C. Chen ${ }^{64}$, H. Chen ${ }^{25}$, K. Chen ${ }^{149}$, L. Chen ${ }^{33 d, i}$, S. Chen ${ }^{33 c}$, X. Chen ${ }^{33 f}$, Y. Chen ${ }^{67}$, H. C. Cheng ${ }^{89}$, Y. Cheng ${ }^{31}$, A. Cheplakov ${ }^{65}$, E. Cheremushkina ${ }^{130}, \quad$ R. Cherkaoui El Moursli ${ }^{136 e}$, V. Chernyatin ${ }^{25, *}$ E. Cheu ${ }^{7}$, L. Chevalier ${ }^{137}$, V. Chiarella ${ }^{47}$, J. T. Childers ${ }^{6}$, G. Chiodini ${ }^{73 a}$, A. S. Chisholm ${ }^{18}$, R. T. Chislett ${ }^{78}$, A. Chitan ${ }^{26 a}$, M. V. Chizhov ${ }^{65}$, K. Choi $^{61}$, S. Chouridou ${ }^{9}$, B. K. B. Chow ${ }^{100}$, V. Christodoulou ${ }^{78}$, D. Chromek-Burckhart ${ }^{30}$, M. L. Chu ${ }^{152}$, J. Chudoba ${ }^{127}$, A. J. Chuinard ${ }^{87}$, J. J. Chwastowski ${ }^{39}$, L. Chytka ${ }^{115}$, G. Ciapetti ${ }^{133 a, 133 b}$, A. K. Ciftci ${ }^{4 a}$, D. Cinca ${ }^{53}$, V. Cindro ${ }^{75}$, I. A. Cioara ${ }^{21}$, A. Ciocio ${ }^{15}$, Z. H. Citron ${ }^{173}$, M. Ciubancan ${ }^{26 a}$, A. Clark ${ }^{49}$, B. L. Clark ${ }^{57}$, P. J. Clark ${ }^{46}$, R. N. Clarke ${ }^{15}$, W. Cleland ${ }^{125}$, C. Clement ${ }^{147 a, 147 b}$, Y. Coadou ${ }^{85}$, M. Cobal ${ }^{165 a, 165 c}$, A. Coccaro ${ }^{139}$, J. Cochran ${ }^{64}$, L. Coffey $^{23}$, J. G. Cogan ${ }^{144}$, B. Cole ${ }^{35}$, S. Cole ${ }^{108}$, A. P. Colijn ${ }^{107}$, J. Collot ${ }^{55}$, T. Colombo ${ }^{58 c}$, G. Compostella ${ }^{101}$, P. Conde Muiño ${ }^{126 a, 126 b}$, E. Coniavitis ${ }^{48}$, S. H. Connell ${ }^{146 b}$, I. A. Connelly 77 , S. M. Consonni ${ }^{91 a}, 91 b$, V. Consorti ${ }^{48}$, S. Constantinescu ${ }^{26 a}$, C. Conta ${ }^{121 a, 121 b}$, G. Conti ${ }^{30}$, F. Conventi ${ }^{104 a, j}$, M. Cooke ${ }^{15}$, B. D. Cooper ${ }^{78}$, A. M. Cooper-Sarkar ${ }^{120}$, K. Copic ${ }^{15}$, T. Cornelissen ${ }^{176}$, M. Corradi ${ }^{20 a}$, F. Corriveau ${ }^{87, k}$, A. Corso-Radu ${ }^{164}$, A. Cortes-Gonzalez ${ }^{12}$, G. Cortiana ${ }^{101}$,

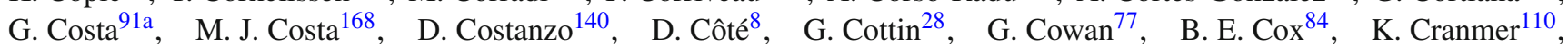
G. Cree $^{29}$, S. Crépé-Renaudin ${ }^{55}$ F. Crescioli ${ }^{80}$, W. A. Cribbs ${ }^{147 a, 147 b}, \quad$ M. Crispin Ortuzar ${ }^{120}$, M. Cristinziani ${ }^{21}$, V. Croft ${ }^{106}$, G. Crosetti ${ }^{37 a, 37 b}$, T. Cuhadar Donszelmann ${ }^{140}$, J. Cummings ${ }^{177}$, M. Curatolo ${ }^{47}$, C. Cuthbert ${ }^{151}$, H. Czirr ${ }^{142}$, P. Czodrowski ${ }^{3}$, S. D’Auria ${ }^{53}$, M. D’Onofrio ${ }^{74}$, M. J. Da Cunha Sargedas De Sousa ${ }^{126 a, 126 b}$, C. Da Via ${ }^{84}$, W. Dabrowski ${ }^{38 a}$, A. Dafinca ${ }^{120}$, T. Dai ${ }^{89}$, O. Dale ${ }^{14}$, F. Dallaire ${ }^{95}$, C. Dallapiccola ${ }^{86}$, M. Dam ${ }^{36}$, J. R. Dandoy ${ }^{31}$, A. C. Daniells ${ }^{18}$, M. Danninger ${ }^{169}$, M. Dano Hoffmann ${ }^{137}$, V. Dao ${ }^{48}$, G. Darbo ${ }^{50 a}$, S. Darmora ${ }^{8}$, J. Dassoulas ${ }^{3}$, A. Dattagupta ${ }^{61}$, W. Davey ${ }^{21}$, C. David ${ }^{170}$, T. Davidek ${ }^{129}$, E. Davies ${ }^{120,1}$, M. Davies ${ }^{154}$, P. Davison ${ }^{78}$, Y. Davygora ${ }^{58 a}$, E. Dawe ${ }^{88}$, I. Dawson ${ }^{140}$, R. K. Daya-Ishmukhametova ${ }^{86}$, K. De ${ }^{8}$ ， R. de Asmundis ${ }^{104 a}$ ， S. De Castro ${ }^{20 a, 20 b}$ ， S. De Cecco ${ }^{80}, \quad$ N. De Groot ${ }^{106}$, P. de Jong ${ }^{107}$, H. De la Torre ${ }^{82}$, F. De Lorenzi ${ }^{64}$, L. De Nooij ${ }^{107}$, D. De Pedis ${ }^{133 a}$, A. De Salvo ${ }^{133 a}$, U. De Sanctis ${ }^{150}$, A. De Santo ${ }^{150}$ ， J. B. De Vivie De Regie ${ }^{117}$ ， W. J. Dearnaley ${ }^{72}$ ， R. Debbe ${ }^{25}$, C. Debenedetti ${ }^{138}$, D. V. Dedovich ${ }^{65}$, I. Deigaard ${ }^{107}$, J. Del Peso ${ }^{82}$, T. Del Prete ${ }^{124 a, 124 b}$, D. Delgove ${ }^{117}$, F. Deliot ${ }^{137}$, C. M. Delitzsch ${ }^{49}$, M. Deliyergiyev ${ }^{75}$, A. Dell'Acqua ${ }^{30}$, L. Dell'Asta ${ }^{22}, \quad$ M. Dell'Orso ${ }^{124 a, 124 b}, \quad$ M. Della Pietra ${ }^{104 a, j}, \quad$ D. della Volpe ${ }^{49}, \quad$ M. Delmastro ${ }^{5}$, P. A. Delsart ${ }^{55}$ ， C. Deluca ${ }^{107}$ ， D. A. DeMarco ${ }^{159}$ ， S. Demers ${ }^{177}$ ， M. Demichev ${ }^{65}$ ， A. Demilly ${ }^{80 ， \quad S . ~ P . ~ D e n i s o v ~}{ }^{130}$, D. Derendarz ${ }^{39}$, J. E. Derkaoui ${ }^{136 d}$, F. Derue ${ }^{80}$, P. Dervan ${ }^{74}$, K. Desch ${ }^{21}$, C. Deterre ${ }^{42}$, P. O. Deviveiros ${ }^{30}$, A. Dewhurst ${ }^{131}$, S. Dhaliwal ${ }^{107}$, A. Di Ciaccio ${ }^{134 a, 134 b}$, L. Di Ciaccio ${ }^{5}$, A. Di Domenico ${ }^{133 a, 133 b}$, C. Di Donato ${ }^{104 a, 104 b}$, A. Di Girolamo ${ }^{30}$, B. Di Girolamo ${ }^{30}$, A. Di Mattia ${ }^{153}$, B. Di Micco ${ }^{135 a, 135 b}$, R. Di Nardo ${ }^{47}$, A. Di Simone ${ }^{48}$, R. Di Sipio ${ }^{159}$, D. Di Valentino ${ }^{29}$, C. Diaconu ${ }^{85}$, M. Diamond ${ }^{159}$, F. A. Dias ${ }^{46}$, M. A. Diaz ${ }^{32 a}$, E. B. Diehl ${ }^{89}$, J. Dietrich ${ }^{16}$, S. Diglio ${ }^{85}$, A. Dimitrievska ${ }^{13}$, J. Dingfelder ${ }^{21}$, P. Dita ${ }^{26 a}$, S. Dita ${ }^{26 a}$,F. Dittus ${ }^{30}$, F. Djama ${ }^{85}$, T. Djobava ${ }^{51 b}$, J. I. Djuvsland ${ }^{58 a}$, M. A. B. do Vale ${ }^{24 c}$, D. Dobos $^{30}$, M. Dobre ${ }^{26 a}$, C. Doglioni ${ }^{49}$, T. Dohmae ${ }^{156}$, J. Dolejsi $^{129}$, Z. Dolezal ${ }^{129}$, B. A. Dolgoshein ${ }^{98, *}$, M. Donadelli ${ }^{24 d}$, S. Donati ${ }^{124 a, 124 b}$, P. Dondero ${ }^{121 a, 121 b}$, J. Donini ${ }^{34}$, J. Dopke ${ }^{131}$, A. Doria ${ }^{104 a}$, M. T. Dova ${ }^{71}$, A. T. Doyle ${ }^{53}$, E. Drechsler ${ }^{54}$, M. Dris ${ }^{10}$, E. Dubreuil ${ }^{34}$, E. Duchovni ${ }^{173}$, G. Duckeck ${ }^{100}$, O. A. Ducu ${ }^{26 a, 85}$, D. Duda ${ }^{176}$, A. Dudarev ${ }^{30}$, L. Duflot ${ }^{117}$, L. Duguid 77 , M. Dührssen ${ }^{30}$, M. Dunford ${ }^{58 a}$, H. Duran Yildiz ${ }^{4 a}$, M. Düren ${ }^{52}$, A. Durglishvili ${ }^{51 b}$, D. Duschinger ${ }^{44}$, M. Dwuznik ${ }^{38 a}$, M. Dyndal ${ }^{38 a}$, C. Eckardt ${ }^{42}$, K. M. Ecker ${ }^{101}$, W. Edson ${ }^{2}$, N. C. Edwards ${ }^{46}$, W. Ehrenfeld ${ }^{21}$, T. Eifert ${ }^{30}$, 
G. Eigen ${ }^{14}$ ，K. Einsweiler ${ }^{15}$ ，T. Ekelof ${ }^{167}$, M. El Kacimi ${ }^{136 c}$, M. Ellert ${ }^{167}$ ， S. Elles ${ }^{5}$, F. Ellinghaus ${ }^{83}$, A. A. Elliot ${ }^{170}$, N. Ellis ${ }^{30}$, J. Elmsheuser ${ }^{100}$, M. Elsing ${ }^{30}$, D. Emeliyanov ${ }^{131}$, Y. Enari ${ }^{156}$, O. C. Endner ${ }^{83}$, M. Endo ${ }^{118}$, R. Engelmann ${ }^{149}$, J. Erdmann ${ }^{43}$, A. Ereditato ${ }^{17}$, G. Ernis ${ }^{176}$, J. Ernst ${ }^{2}$, M. Ernst ${ }^{25}$, S. Errede ${ }^{166}$, E. Ertel ${ }^{83}$, M. Escalier ${ }^{117}$, H. Esch ${ }^{43}$, C. Escobar ${ }^{125}$, B. Esposito ${ }^{47}$, A. I. Etienvre ${ }^{137}$, E. Etzion ${ }^{154}$, H. Evans ${ }^{61}$, A. Ezhilov ${ }^{123}$, L. Fabbri20a,20b, G. Facini ${ }^{31}$, R. M. Fakhrutdinov ${ }^{130}$, S. Falciano ${ }^{133 a}$, R. J. Falla ${ }^{78}$, J. Faltova ${ }^{129}$, Y. Fang ${ }^{33 a}$, M. Fanti ${ }^{91 a, 91 b}$, A. Farbin ${ }^{8}$, A. Farilla ${ }^{135 a}$, T. Farooque ${ }^{12}$, S. Farrell ${ }^{15}, \quad$ S. M. Farrington ${ }^{171}, \quad$ P. Farthouat ${ }^{30}, \quad$ F. Fassi ${ }^{136 e}$, P. Fassnacht ${ }^{30}$, D. Fassouliotis ${ }^{9}$, A. Favareto ${ }^{50 a, 50 b}$, L. Fayard ${ }^{117}$, P. Federic ${ }^{145 a}$, O. L. Fedin ${ }^{123, m}$, W. Fedorko ${ }^{169}$, S. Feig1 ${ }^{30}$, L. Feligioni ${ }^{85}$, C. Feng ${ }^{33 d}$, E. J. Feng ${ }^{6}$, H. Feng ${ }^{89}$, A. B. Fenyuk ${ }^{130}$, P. Fernandez Martinez ${ }^{168}$, S. Fernandez Perez ${ }^{30}$ ， S. Ferrag ${ }^{53}$, J. Ferrando ${ }^{53}$,

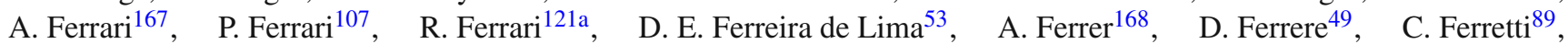
A. Ferretto Parodi ${ }^{50 a, 50 b}$, M. Fiascaris ${ }^{31}$, F. Fiedler ${ }^{83}$, A. Filipčič ${ }^{75}$, M. Filipuzzi ${ }^{42}$, F. Filthaut ${ }^{106}$, M. Fincke-Keeler ${ }^{170}$, K. D. Finelli ${ }^{151}$, M. C. N. Fiolhais ${ }^{126 a, 126 c}$, L. Fiorini ${ }^{168}$, A. Firan ${ }^{40}$, A. Fischer ${ }^{2}$, C. Fischer ${ }^{12}$, J. Fischer ${ }^{176}$, W. C. Fisher ${ }^{90}$, E. A. Fitzgerald ${ }^{23}$, M. Flechl ${ }^{48}$, I. Fleck ${ }^{142}$, P. Fleischmann ${ }^{89}$, S. Fleischmann ${ }^{176}$, G. T. Fletcher ${ }^{140}$, G. Fletcher ${ }^{76}$, T. Flick ${ }^{176}$, A. Floderus ${ }^{81}$, L. R. Flores Castillo ${ }^{60 a}$, M. J. Flowerdew ${ }^{101}$, A. Formica ${ }^{137}$, A. Forti ${ }^{84}$, D. Fournier ${ }^{117}$, H. Fox ${ }^{72}$, S. Fracchia ${ }^{12}$, P. Francavilla ${ }^{80}$, M. Franchini ${ }^{20 a, 20 b}$, D. Francis ${ }^{30}$, L. Franconi ${ }^{119}$, M. Franklin ${ }^{57}$, M. Fraternali ${ }^{121 a, 121 b}$, D. Freeborn $^{78}$, S. T. French ${ }^{28}$, F. Friedrich ${ }^{44}$, D. Froidevaux ${ }^{30}$, J. A. Frost ${ }^{120}$, C. Fukunaga ${ }^{157}$, E. Fullana Torregrosa ${ }^{83}$, B. G. Fulsom ${ }^{144}$, J. Fuster ${ }^{168}$, C. Gabaldon ${ }^{55}$, O. Gabizon ${ }^{176}$, A. Gabrielli20a,20b, A. Gabrielli ${ }^{133 a, 133 b}$, S. Gadatsch ${ }^{107}$, S. Gadomski ${ }^{49}$, G. Gagliardi ${ }^{50 a, 50 b}$, P. Gagnon ${ }^{61}$, C. Galea ${ }^{106}$, B. Galhardo ${ }^{126 a, 126 c}$, E. J. Gallas ${ }^{120}$, B. J. Gallop ${ }^{131}$, P. Gallus ${ }^{128}$, G. Galster ${ }^{36}$, K. K. Gan ${ }^{111}$, J. Gao ${ }^{33 b, 85}$, Y. Gao ${ }^{46}$, Y. S. Gao ${ }^{144, e}$, F. M. Garay Walls ${ }^{46}$, F. Garberson ${ }^{177}$, C. García ${ }^{168}$, J. E. García Navarro ${ }^{168}$, M. Garcia-Sciveres ${ }^{15}$, R. W. Gardner ${ }^{31}$, N. Garelli ${ }^{144}$, V. Garonne ${ }^{119}$, C. Gatti ${ }^{47}$, A. Gaudiello50a,50b, G. Gaudio ${ }^{121 a}$, B. Gaur ${ }^{142}$, L. Gauthier ${ }^{95}$, P. Gauzzi133a,133b, I. L. Gavrilenko ${ }^{96}$, C. Gay ${ }^{169}$, G. Gaycken $^{21}$ ， E. N. Gazis ${ }^{10}$ ， P. Ge ${ }^{33 d}$, Z. Gecse ${ }^{169}, \quad$ C. N. P. Gee ${ }^{131}, \quad$ D. A. A. Geerts ${ }^{107}, \quad$ Ch. Geich-Gimbel ${ }^{21}$,

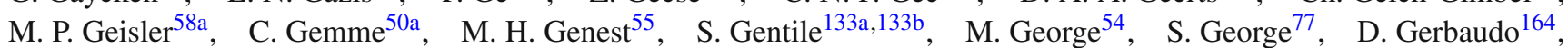
A. Gershon ${ }^{154}$, H. Ghazlane ${ }^{136 b}$, N. Ghodbane ${ }^{34}$, B. Giacobbe ${ }^{20 a}$, S. Giagu ${ }^{133 a, 133 b}$, V. Giangiobbe ${ }^{12}$, P. Giannetti ${ }^{124 a, 124 b}$, B. Gibbard ${ }^{25}$, S. M. Gibson ${ }^{77}$, M. Gilchriese ${ }^{15}$, T. P. S. Gillam ${ }^{28}$, D. Gillberg ${ }^{30}$, G. Gilles ${ }^{34}$, D. M. Gingrich ${ }^{3, d}$, N. Giokaris ${ }^{9}$, M. P. Giordani ${ }^{165 a}, 165$ c , F. M. Giorgi ${ }^{20 a}$, F. M. Giorgi ${ }^{16}$, P. F. Giraud ${ }^{137}$, P. Giromini ${ }^{47}$, D. Giugni ${ }^{91 a}$, C. Giuliani ${ }^{48}$, M. Giulini ${ }^{58 b}$, B. K. Gjelsten ${ }^{119}$, S. Gkaitatzis ${ }^{155}$, I. Gkialas ${ }^{155}$, E. L. Gkougkousis ${ }^{117}$, L. K. Gladilinn ${ }^{99}$, C. Glasman ${ }^{82}$, J. Glatzer ${ }^{30}$, P. C. F. Glaysher ${ }^{46}$, A. Glazov ${ }^{42}$, M. Goblirsch-Kolb ${ }^{101}$, J. R. Goddard ${ }^{76}$, J. Godlewski ${ }^{39}$, S. Goldfarb ${ }^{89}$, T. Golling ${ }^{49}$, D. Golubkov ${ }^{130}$, A. Gomes ${ }^{126 a, 126 b, 126 d,} \quad$ R. Gonçalo ${ }^{126 a}$, J. Goncalves Pinto Firmino Da Costa ${ }^{137}$, L. Gonella ${ }^{21}$, S. González de la Hoz ${ }^{168}$, G. Gonzalez Parra ${ }^{12}$, S. Gonzalez-Sevilla ${ }^{49}$, L. Goossens ${ }^{30}$, P. A. Gorbounov ${ }^{97}$,

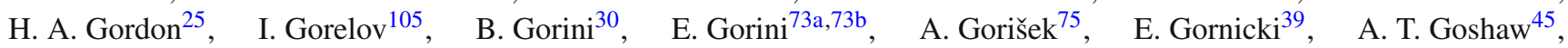
C. Gössling ${ }^{43}$, M. I. Gostkin ${ }^{65}$, D. Goujdami ${ }^{136 c}$, A. G. Goussiou ${ }^{139}$, N. Govender ${ }^{146 b}$, H. M. X. Grabas ${ }^{138}$, L. Graber ${ }^{54}$, I. Grabowska-Bold ${ }^{38 a}$, P. Grafström ${ }^{20 a, 20 b}$, K-J. Grahn ${ }^{42}$, J. Gramling ${ }^{49}$, E. Gramstad ${ }^{119}$, S. Grancagnolo ${ }^{16}$, V. Grassi ${ }^{149}$, V. Gratchev ${ }^{123}$, H. M. Gray ${ }^{30}$, E. Graziani ${ }^{135 a}$, Z. D. Greenwood ${ }^{79, n}$, K. Gregersen ${ }^{78}$, I. M. Gregor ${ }^{42}$, P. Grenier ${ }^{144}$, J. Griffiths ${ }^{8}$, A. A. Grillo ${ }^{138}$, K. Grimm ${ }^{72}$, S. Grinstein ${ }^{12, o}$, Ph. Gris ${ }^{34}$, J.-F. Grivaz ${ }^{117}$, J. P. Grohs ${ }^{44}$, A. Grohsjean ${ }^{42}$, E. Gross ${ }^{173}$, J. Grosse-Knetter ${ }^{54}$, G. C. Grossi ${ }^{79}$, Z. J. Grout ${ }^{150}$, L. Guan ${ }^{33 b}$, J. Guenther ${ }^{128}$, F. Guescini ${ }^{49}$, D. Guest ${ }^{177}$, O. Gueta $^{154}$, E. Guido ${ }^{50 a, 50 b}$, T. Guillemin ${ }^{117}, \quad$ S. Guindon ${ }^{2}$, U. Gul ${ }^{53}, \quad$ C. Gumpert ${ }^{44}$, J. Guo ${ }^{33 e}$, S. Gupta ${ }^{120}$, P. Gutierrez ${ }^{113}$, N. G. Gutierrez Ortiz ${ }^{53}$, C. Gutschow ${ }^{44}$, C. Guyot ${ }^{137}$, C. Gwenlan ${ }^{120}$, C. B. Gwilliam ${ }^{74}$, A. Haas ${ }^{110}$, C. Haber ${ }^{15}$, H. K. Hadavand ${ }^{8}$, N. Haddad ${ }^{136 e}$, P. Haefner ${ }^{21}$, S. Hageböck ${ }^{21}$, Z. Hajduk ${ }^{39}$, H. Hakobyan ${ }^{178}$, M. Haleem ${ }^{42}$, J. Haley ${ }^{114}$, D. Hall ${ }^{120}$, G. Halladjian ${ }^{90}$, G. D. Hallewell ${ }^{85}$, K. Hamacher ${ }^{176}$, P. Hamal ${ }^{115}$, K. Hamano ${ }^{170}$, M. Hamer ${ }^{54}$, A. Hamilton ${ }^{146 a}$ ， S. Hamilton ${ }^{162}$ ， G. N. Hamity ${ }^{146 c}$ ， P. G. Hamnett ${ }^{42}$ ， L. Han ${ }^{33 b}$ ， K. Hanagaki ${ }^{118}$ ， K. Hanawa ${ }^{156}$, M. Hance ${ }^{15}$, P. Hanke ${ }^{58 a}$ ， R. Hanna ${ }^{137}$ ， J. B. Hansen ${ }^{36}$, J. D. Hansen ${ }^{36}$, M. C. Hansen ${ }^{21}$, P. H. Hansen ${ }^{36}$, K. Hara ${ }^{161}$,

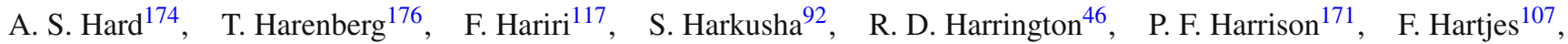
M. Hasegawa ${ }^{67}$, S. Hasegawa ${ }^{103}$, Y. Hasegawa ${ }^{141}$, A. Hasib ${ }^{113}$, S. Hassani ${ }^{137}$, S. Haug ${ }^{17}$, R. Hauser ${ }^{90}$, L. Hauswald ${ }^{44}$, M. Havranek ${ }^{127}$, C. M. Hawkes ${ }^{18}$, R. J. Hawkings ${ }^{30}$, A. D. Hawkins ${ }^{81}$, T. Hayashi ${ }^{161}$, D. Hayden ${ }^{90}$, C. P. Hays ${ }^{120}$, J. M. Hays ${ }^{76}$, H. S. Hayward ${ }^{74}$ ， S. J. Haywood ${ }^{131}$ ， S. J. Head ${ }^{18}$, T. Heck ${ }^{83}$, V. Hedberg ${ }^{81}$, L. Heelan ${ }^{8}$, S. Heim ${ }^{122}$, T. Heim ${ }^{176}$, B. Heinemann ${ }^{15}$, L. Heinrich ${ }^{110}$, J. Hejbal ${ }^{127}$, L. Helary ${ }^{22}$, S. Hellman ${ }^{147 a, 147 b}$, D. Hellmich ${ }^{21}$, C. Helsens ${ }^{30}$, $\begin{array}{lll}\text { J. Henderson } & 120 & \text { R. C. W. Henderson }\end{array}{ }^{72}$, Y. Heng ${ }^{174}$, C. Hengler ${ }^{42}$, A. Henrichs ${ }^{177}$, A. M. Henriques Correia ${ }^{30}$, S. Henrot-Versille ${ }^{117}$, G. H. Herbert ${ }^{16}$, Y. Hernández Jiménez ${ }^{168}$, R. Herrberg-Schubert ${ }^{16}$, G. Herten $^{48}$, R. Hertenberger ${ }^{100}$, L. Hervas $^{30}$ ， G. G. Hesketh ${ }^{78}$ ， N. P. Hessey ${ }^{107}$ ， J. W. Hetherly ${ }^{40}$ ， R. Hickling ${ }^{76}$ ， E. Higón-Rodriguez ${ }^{168}$ ， E. Hill ${ }^{170}$, J. C. Hill ${ }^{28}$, K. H. Hiller ${ }^{42}$, S. J. Hillier ${ }^{18}$, I. Hinchliffe ${ }^{15}$, E. Hines ${ }^{122}$, R. R. Hinman ${ }^{15}$, M. Hirose ${ }^{158}$, D. Hirschbuehl ${ }^{176}$, J. Hobbs ${ }^{149}$ ， N. Hod $^{107}$ ， M. C. Hodgkinson ${ }^{140}$ ， P. Hodgson ${ }^{140}$ ， A. Hoecker ${ }^{30}$ ， M. R. Hoeferkamp ${ }^{105}$ ， F. Hoenig ${ }^{100}$, M. Hohlfeld ${ }^{83}$, D. Hohn ${ }^{21}$, T. R. Holmes ${ }^{15}$, T. M. Hong ${ }^{122}$, L. Hooft van Huysduynen ${ }^{110}$, W. H. Hopkins ${ }^{116}$, Y. Horii ${ }^{103}$, A. J. Horton ${ }^{143}$ ， J-Y. Hostachy ${ }^{55} ，$ S. Hou ${ }^{152} ，$ A. Hoummada ${ }^{136 a}$ ， J. Howard ${ }^{120}$ ， J. Howarth ${ }^{42}$, M. Hrabovsky ${ }^{115}$, 
I. Hristova ${ }^{16}$, J. Hrivnac ${ }^{117}$, T. Hryn'ova ${ }^{5}$, A. Hrynevich ${ }^{93}$, C. Hsu ${ }^{146 c}$, P. J. Hsu ${ }^{152, p}$, S.-C. Hsu ${ }^{139}$, D. Hu ${ }^{35}$, Q. Hu ${ }^{33 b}$, X. Hu ${ }^{89}$, Y. Huang ${ }^{42}$, Z. Hubacek ${ }^{30}$ ，F. Hubaut ${ }^{85}$, F. Huegging ${ }^{21}$, T. B. Huffman ${ }^{120}$, E. W. Hughes ${ }^{35}$, G. Hughes ${ }^{72}$,

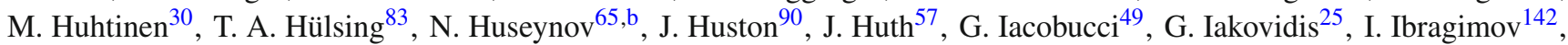
L. Iconomidou-Fayard ${ }^{117}$, E. Ideal ${ }^{177}$, Z. Idrissi ${ }^{136 e}$, P. Iengo ${ }^{30}$, O. Igonkina ${ }^{107}$, T. Iizawa ${ }^{172}$, Y. Ikegami ${ }^{66}$, K. Ikematsu $^{142}$, M. Ikeno ${ }^{66}$, Y. Ilchenko ${ }^{31, q}$, D. Iliadis ${ }^{155}$, N. Ilic ${ }^{159}$, Y. Inamaru ${ }^{67}$, T. Ince ${ }^{101}$, P. Ioannou ${ }^{9}$, M. Iodice ${ }^{135 a}$, K. Iordanidou ${ }^{9}$, V. Ippolito ${ }^{57}$, A. Irles Quiles ${ }^{168}$, C. Isaksson ${ }^{167}$, M. Ishino ${ }^{68}$, M. Ishitsuka ${ }^{158}$ ， R. Ishmukhametov ${ }^{111}$, C. Issever ${ }^{120}$, S. Istin ${ }^{19 a}$, J. M. Iturbe Ponce ${ }^{84}$, R. Iuppa ${ }^{134 a, 134 b}$, J. Ivarsson ${ }^{81}$, W. Iwanski ${ }^{39}$, H. Iwasaki ${ }^{66}$, J. M. Izen ${ }^{41}$, V. Izzo ${ }^{104 a}$, S. Jabbar ${ }^{3}$, B. Jackson ${ }^{122}$, M. Jackson ${ }^{74}$, P. Jackson ${ }^{1}$, M. R. Jaekel ${ }^{30}$, V. Jain ${ }^{2}$, K. Jakobs ${ }^{48}$, S. Jakobsen ${ }^{30}$, T. Jakoubek ${ }^{127}$, J. Jakubek ${ }^{128}$ ， D. O. Jamin ${ }^{152}$ ， D. K. Jana ${ }^{79}$ ，E. Jansen ${ }^{78}$ ， R. W. Jansky ${ }^{62}$ ，J. Janssen ${ }^{21}$ ， M. Janus ${ }^{171}$ ， G. Jarlskog ${ }^{81}$,

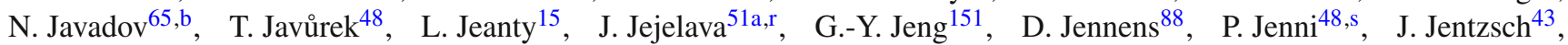

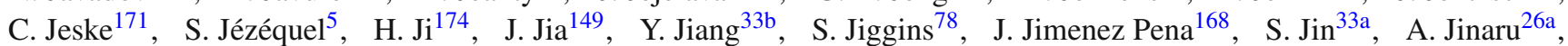
O. Jinnouchi ${ }^{158}$, M. D. Joergensen ${ }^{36}$, P. Johansson ${ }^{140}$, K. A. Johns ${ }^{7}$, K. Jon-And ${ }^{147 a, 147 b}$, G. Jones ${ }^{171}$, R. W. L. Jones ${ }^{72}$, T. J. Jones ${ }^{74}$ ，J. Jongmanns ${ }^{58 a}$ ，P. M. Jorge ${ }^{126 a, 126 b}$, K. D. Joshi ${ }^{84}$ ，J. Jovicevic ${ }^{160 a} ， X$. Ju$^{174} ，$ C. A. Jung ${ }^{43}$ ，P. Jussel ${ }^{62}$, A. Juste Rozas ${ }^{12,0}$, M. Kaci ${ }^{168}$, A. Kaczmarska ${ }^{39}$, M. Kado ${ }^{117}$, H. Kagan ${ }^{111}$, M. Kagan ${ }^{144}$, S. J. Kahn ${ }^{85}$, E. Kajomovitz ${ }^{45}$, C. W. Kalderon ${ }^{120}$, S. Kama ${ }^{40}$, A. Kamenshchikov ${ }^{130}$, N. Kanaya ${ }^{156}$, M. Kaneda ${ }^{30}$, S. Kaneti ${ }^{28}$, V. A. Kantserov ${ }^{98}$, J. Kanzaki ${ }^{66}$, B. Kaplan ${ }^{110}$, A. Kapliy ${ }^{31}$, D. Kar ${ }^{53}$, K. Karakostas ${ }^{10}$, A. Karamaoun ${ }^{3}$, N. Karastathis ${ }^{10,107}$, M. J. Kareem4, M. Karnevskiy ${ }^{83}$, S. N. Karpov ${ }^{65}$, Z. M. Karpova ${ }^{65}$, K. Karthik ${ }^{110}$, V. Kartvelishvilii2, A. N. Karyukhin ${ }^{130}$, L. Kashif ${ }^{174}$, R. D. Kass ${ }^{111}$, A. Kastanas ${ }^{14}$, Y. Kataoka ${ }^{156}$, A. Katre ${ }^{49}$, J. Katzy ${ }^{42}$, K. Kawagoe ${ }^{70}$, T. Kawamoto ${ }^{156}$, G. Kawamura ${ }^{54}$, S. Kazama ${ }^{156}$, V. F. Kazanin ${ }^{109, c}$, M. Y. Kazarinov ${ }^{65}$, R. Keeler ${ }^{170}$, R. Kehoe ${ }^{40}$, J. S. Keller ${ }^{42}$, J. J. Kempster ${ }^{77}$, H. Keoshkerian ${ }^{84}$, O. Kepka ${ }^{127}$, B. P. Kerševan ${ }^{75}$, S. Kersten ${ }^{176}$, R. A. Keyes ${ }^{87}$, F. Khalil-zada ${ }^{11}$, H. Khandanyan ${ }^{147 a, 147 b}$, A. Khanov ${ }^{114}$, A. G. Kharlamov ${ }^{109, c}$, T. J. Khoo $^{28}$, V. Khovanskiy ${ }^{97}$, E. Khramov ${ }^{65}$, J. Khubua ${ }^{51 b, t}$, H. Y. Kim ${ }^{8}$, H. Kim ${ }^{147 a, 147 b}$, S. H. Kim ${ }^{161}$, Y. Kim ${ }^{31}$, N. Kimura ${ }^{155}$, O. M. Kind ${ }^{16}$, B. T. King ${ }^{74}$, M. King ${ }^{168}$, R. S. B. King ${ }^{120}$, S. B. King ${ }^{169}$, J. Kirk ${ }^{131}$, A. E. Kiryunin ${ }^{101}$, T. Kishimoto ${ }^{67}$, D. Kisielewska ${ }^{38 a}$, F. Kiss ${ }^{48}$, K. Kiuchi ${ }^{161}$, O. Kivernyk ${ }^{137}$, E. Kladiva ${ }^{145 b}$, M. H. Klein ${ }^{35}$, M. Klein ${ }^{74}$, U. Klein ${ }^{74}$, K. Kleinknecht ${ }^{83}$, P. Klimek ${ }^{147 a, 147 b}$, A. Klimentov ${ }^{25}$, R. Klingenberg ${ }^{43}$ ， J. A. Klinger ${ }^{84}$ ， T. Klioutchnikova ${ }^{30}$ ， P. F. Klok ${ }^{106}$ ， E.-E. Kluge ${ }^{58 a} ， \quad$ P. Kluit ${ }^{107}, \quad$ S. Kluth ${ }^{101}$, E. Kneringer ${ }^{62}$, E. B. F. G. Knoops ${ }^{85}$, A. Knue ${ }^{53}$, D. Kobayashi ${ }^{158}$, T. Kobayashi ${ }^{156}$, M. Kobel ${ }^{44}$, M. Kocian ${ }^{144}$, P. Kodys ${ }^{129}$, T. Koffas ${ }^{29}$, E. Koffeman ${ }^{107}$, L. A. Kogan ${ }^{120}$, S. Kohlmann ${ }^{176}$, Z. Kohout ${ }^{128}$, T. Kohriki ${ }^{66}$, T. Koi ${ }^{144}$, H. Kolanoski ${ }^{16}$, I. Koletsou ${ }^{5}$, A. A. Komar ${ }^{96, *}$, Y. Komori ${ }^{156}$, T. Kondo ${ }^{66}$, N. Kondrashova ${ }^{42}$, K. Köneke ${ }^{48}$, A. C. König ${ }^{106}$, S. König ${ }^{83}$, T. Kono ${ }^{66, u}$, R. Konoplich ${ }^{110, v}$, N. Konstantinidis ${ }^{78}$, R. Kopeliansky ${ }^{153}$, S. Koperny ${ }^{38 a}$, L. Köpke ${ }^{83}$, A. K. Kopp ${ }^{48}$, K. Korcyl1 ${ }^{39}$, K. Kordas ${ }^{155}$, A. $\operatorname{Korn}^{78}$, A. A. Korol ${ }^{109, c}$, I. Korolkov ${ }^{12}$, E. V. Korolkova ${ }^{140}$, O. Kortner ${ }^{101}$, S. Kortner ${ }^{101}$, T. Kosek ${ }^{129}$, V. V. Kostyukhin ${ }^{21}$, V. M. Kotov ${ }^{65}$, A. Kotwal ${ }^{45}$, A. Kourkoumeli-Charalampidi ${ }^{155}$, $\begin{array}{lll}\text { C. Kourkoumelis } & \end{array}$, V. Kouskoura ${ }^{25}$, A. Koutsman ${ }^{160 a}$, R. Kowalewski ${ }^{170,}$ T. Z. Kowalski ${ }^{38 a}$, W. Kozanecki ${ }^{137}$, A. S. Kozhin ${ }^{130}$, V. A. Kramarenko ${ }^{99}$, G. Kramberger ${ }^{75}$, D. Krasnopevtsev ${ }^{98}$, A. Krasznahorkay ${ }^{30}$, J. K. Kraus ${ }^{21}$, A. Kravchenko ${ }^{25}$, S. Kreiss ${ }^{110}$, M. Kretz ${ }^{58 c}$, J. Kretzschmar ${ }^{74}$, K. Kreutzfeldt ${ }^{52}$, P. Krieger ${ }^{159}$, K. Krizka ${ }^{31}$, K. Kroeninger ${ }^{43}$, H. Kroha ${ }^{101}$, J. Kroll ${ }^{122}$, J. Kroseberg ${ }^{21}$, J. Krstic ${ }^{13}$, U. Kruchonak ${ }^{65}$, H. Krüger ${ }^{21}$, N. Krumnack ${ }^{64}$, Z. V. Krumshteyn ${ }^{65}$, A. Kruse ${ }^{174}$, M. C. Kruse ${ }^{45}$, M. Kruskal ${ }^{22}$, T. Kubota ${ }^{88}$, H. Kucuk ${ }^{78}$, S. Kuday ${ }^{4 c}$, S. Kuehn ${ }^{48}$, A. Kugel ${ }^{58 c}$, F. Kuger ${ }^{175}$, A. Kuhl ${ }^{138}$, T. Kuhl ${ }^{42}$, V. Kukhtin ${ }^{65}$, Y. Kulchitsky ${ }^{92}$, S. Kuleshov ${ }^{32 b}$, M. Kuna ${ }^{133 a, 133 b}$, T. Kunigo ${ }^{68}$, A. Kupco ${ }^{127}$, H. Kurashige ${ }^{67}$, Y. A. Kurochkin ${ }^{92}$, R. Kurumida ${ }^{67}$, V. Kus ${ }^{127}$, E. S. Kuwertz ${ }^{148}$, M. Kuze ${ }^{158}$, J. Kvita ${ }^{115}$, T. Kwan ${ }^{170}$, D. Kyriazopoulos ${ }^{140}$, A. La Rosa ${ }^{49}$, J. L. La Rosa Navarro ${ }^{24 d}$, L. La Rotonda ${ }^{37 a, 37 b}$, C. Lacasta ${ }^{168}$, F. Lacava ${ }^{133 a, 133 b}$, J. Lacey ${ }^{29}$, H. Lacker ${ }^{16}$, D. Lacour ${ }^{80}$, V. R. Lacuesta ${ }^{168}$, E. Ladygin ${ }^{65}$, R. Lafaye ${ }^{5}$, B. Laforge ${ }^{80}$, T. Lagouri ${ }^{177}$, S. Lai ${ }^{48}$, L. Lambourne ${ }^{78}$, S. Lammers ${ }^{61}$, C. L. Lampen ${ }^{7}$, W. Lampl ${ }^{7}$, E. Lançon ${ }^{137}$, U. Landgraf ${ }^{48}$, M. P. J. Landon ${ }^{76}$, V. S. Lang ${ }^{58 a}$, J. C. Lange ${ }^{12}$, A. J. Lankford ${ }^{164}$, F. Lanni ${ }^{25}$, K. Lantzsch ${ }^{30}$, S. Laplace ${ }^{80}$, C. Lapoire ${ }^{30}$, J. F. Laporte ${ }^{137}$, T. Lari ${ }^{91 a}$, F. Lasagni Manghi'20a,20b, M. Lassnig ${ }^{30}$, P. Laurelli ${ }^{47}$, W. Lavrijsen ${ }^{15}$, A. T. Law ${ }^{138}$, P. Laycock ${ }^{74}$, O. Le Dortz ${ }^{80}$, E. Le Guirriec ${ }^{85}$, E. Le Menedeu ${ }^{12}$, M. LeBlanc ${ }^{170}$, T. LeCompte ${ }^{6}$, F. Ledroit-Guillon ${ }^{55}$, C. A. Lee ${ }^{146 b}$, S. C. Lee ${ }^{152}$, L. Lee ${ }^{1}$, G. Lefebvre ${ }^{80}$, M. Lefebvre ${ }^{170}$, F. Legger ${ }^{100}$, C. Leggett ${ }^{15}$, A. Lehan ${ }^{74}$, G. Lehmann Miotto ${ }^{30}$, X. Lei ${ }^{7}$, W. A. Leight ${ }^{29}$, A. Leisos ${ }^{155}$, A. G. Leister ${ }^{177}, \quad$ M. A. L. Leite ${ }^{24 d}, \quad$ R. Leitner ${ }^{129}$, D. Lellouch ${ }^{173}, \quad$ B. Lemmer $^{54}$, K. J. C. Leney ${ }^{78}$, T. Lenz ${ }^{21}$, B. Lenzi ${ }^{30}$, R. Leone ${ }^{7}$, S. Leone ${ }^{124 a, 124 b}$, C. Leonidopoulos ${ }^{46}$, S. Leontsinis ${ }^{10}$, C. Leroy ${ }^{95}$, C. G. Lester $^{28}$, M. Levchenko ${ }^{123}$, J. Levêque ${ }^{5}$, D. Levin ${ }^{89}$, L. J. Levinson ${ }^{173}$, M. Levy ${ }^{18}$, A. Lewis ${ }^{120}$, A. M. Leyko ${ }^{21}$,

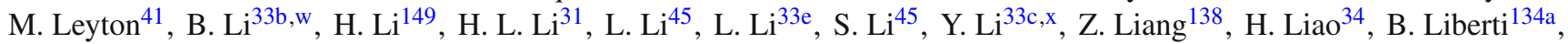
A. Liblong ${ }^{159}$, P. Lichard ${ }^{30}$, K. Lie ${ }^{166}$, J. Liebal ${ }^{21}$, W. Liebig ${ }^{14}$, C. Limbach ${ }^{21}$, A. Limosani151, S. C. Lin ${ }^{152, y}$, T. H. Lin ${ }^{83}$, F. Linde ${ }^{107}$, B. E. Lindquist ${ }^{149}$, J. T. Linnemann ${ }^{90}$, E. Lipeles ${ }^{122}$, A. Lipniacka ${ }^{14}$, M. Lisovyi ${ }^{42}$, T. M. Liss ${ }^{166}$, D. Lissauer ${ }^{25}$,

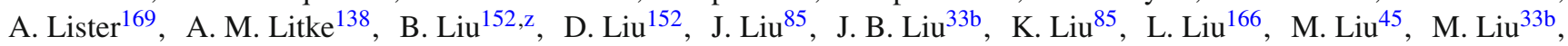
Y. Liu ${ }^{33 b}$, M. Livan ${ }^{121 a, 121 b}$, A. Lleres ${ }^{55}$, J. Llorente Merino ${ }^{82}$, S. L. Lloyd $d^{76}$, F. Lo Sterzo ${ }^{152}$, E. Lobodzinska ${ }^{42}$, 
P. Loch ${ }^{7}$, W. S. Lockman ${ }^{138}$, F. K. Loebinger ${ }^{84}$, A. E. Loevschall-Jensen ${ }^{36}$, A. Loginov ${ }^{177}$, T. Lohse ${ }^{16}$, K. Lohwasser ${ }^{42}$, M. Lokajicek ${ }^{127}$, B. A. Long ${ }^{22}$, J. D. Long ${ }^{89}$, R. E. Long ${ }^{72}$, K. A. Looper ${ }^{111}$, L. Lopes ${ }^{126 a}$, D. Lopez Mateos ${ }^{57}$, B. Lopez Paredes ${ }^{140}$, I. Lopez Paz ${ }^{12}$, J. Lorenz ${ }^{100}$, N. Lorenzo Martinez ${ }^{61}$, M. Losada ${ }^{163}$, P. Loscutoff ${ }^{15}$, P. J. Lösel ${ }^{100}$, X. Lou ${ }^{33 a}$, A. Lounis ${ }^{117}$, J. Love ${ }^{6}$, P. A. Love ${ }^{72}$, N. Lu ${ }^{89}$, H. J. Lubatti ${ }^{139}$, C. Luci 133a,133b , A. Lucotte ${ }^{55}$, F. Luehring ${ }^{61}$, W. Lukas ${ }^{62}$, L. Luminari ${ }^{133 a}$, O. Lundberg ${ }^{147 a, 147 b}$, B. Lund-Jensen ${ }^{148}$, M. Lungwitz ${ }^{83}$, D. Lynn ${ }^{25}$, R. Lysak ${ }^{127}$, E. Lytken ${ }^{81}$, H. $\mathrm{Ma}^{25}$, L. L. Ma ${ }^{33 \mathrm{~d}}$, G. Maccarrone ${ }^{47}$, A. Macchiolo ${ }^{101}$, C. M. Macdonald ${ }^{140}$, J. Machado Miguens ${ }^{122,126 b}$, D. Macina ${ }^{30}$, D. Madaffari ${ }^{85}$, R. Madar ${ }^{34}$, H. J. Maddocks ${ }^{72}$, W. F. Mader ${ }^{44}$, A. Madsen ${ }^{167}$, S. Maeland ${ }^{14}$, T. Maeno ${ }^{25}$, A. Maevskiy ${ }^{99}$, E. Magradze ${ }^{54}$, K. Mahboubi ${ }^{48}$ ， J. Mahlstedt ${ }^{107}$, C. Maiani ${ }^{137}$ ， C. Maidantchik ${ }^{24 a}$ ， A. A. Maier ${ }^{101}$, T. Maier ${ }^{100}$, A. Maio ${ }^{126 a, 126 b, 126 d}$, S. Majewski ${ }^{116}$, Y. Makida ${ }^{66}$, N. Makovec ${ }^{117}$, B. Malaescu ${ }^{80}$, Pa. Malecki ${ }^{39}$, V. P. Maleev ${ }^{123}$, F. Malek ${ }^{55}$, U. Mallik ${ }^{63}$, D. Malon ${ }^{6}$, C. Malone ${ }^{144}$, S. Maltezos ${ }^{10}$, V. M. Malyshev ${ }^{109}$, S. Malyukov ${ }^{30}$, J. Mamuzic ${ }^{42}$, G. Mancini ${ }^{47}$, B. Mandelli30, L. Mandelli ${ }^{91 a}$, I. Mandić ${ }^{75}$ ，R. Mandrysch ${ }^{63}$ ，J. Maneira ${ }^{126 a, 126 b}$, A. Manfredini ${ }^{101}$, L. Manhaes de Andrade Filho ${ }^{24 b}$, J. Manjarres Ramos ${ }^{160 b}$, A. Mann ${ }^{100}$, P. M. Manning ${ }^{138}$, A. Manousakis-Katsikakis9, B. Mansoulie ${ }^{137}$, R. Mantifel ${ }^{87}$, M. Mantoani ${ }^{54}$, L. Mapelli ${ }^{30}$, L. March ${ }^{146 c}$, G. Marchiori ${ }^{80}$, M. Marcisovsky ${ }^{127}$, C. P. Marino ${ }^{170}$, M. Marjanovic ${ }^{13}$, F. Marroquim ${ }^{24 a}$, S. P. Marsden ${ }^{84}$, Z. Marshall ${ }^{15}$, L. F. Marti ${ }^{17}$, S. Marti-Garcia ${ }^{168}$, B. Martin ${ }^{90}$, T. A. Martin ${ }^{171}$, V. J. Martin ${ }^{46}$, B. Martin dit Latour ${ }^{14}$, M. Martinez ${ }^{12, o}$, S. Martin-Haugh ${ }^{131}$, V. S. Martoiu ${ }^{26 a}$, A. C. Martyniuk ${ }^{78}$, M. Marx ${ }^{139}$, F. Marzano ${ }^{133 a}$, A. Marzin $^{30}$, L. Masetti ${ }^{83}$, T. Mashimo ${ }^{156}$, R. Mashinistov ${ }^{96}$, J. Masik ${ }^{84}$, A. L. Maslennikov ${ }^{109, c}$, I. Massa ${ }^{20 a, 20 b}$ ， L. Massa ${ }^{20 a, 20 b}$ ， N. Massol ${ }^{5}$ ，P. Mastrandrea ${ }^{149} ，$ A. Mastroberardino ${ }^{37 a, 37 b}$, T. Masubuchi ${ }^{156}$, P. Mättig ${ }^{176}$ ， J. Mattmann ${ }^{83}$ ， J. Maurer ${ }^{26 a}$ ， S. J. Maxfield ${ }^{74}$ ， D. A. Maximov ${ }^{109, c}$, R. Mazini ${ }^{152}$, S. M. Mazza ${ }^{91 a, 91 b}$, L. Mazzaferro ${ }^{134 a, 134 b}$, G. Mc Goldrick ${ }^{159}$, S. P. Mc Kee ${ }^{89}$, A. McCarn ${ }^{89}$, R. L. McCarthy ${ }^{149}$, T. G. McCarthy ${ }^{29}$, N. A. McCubbin ${ }^{131}$, K. W. McFarlane ${ }^{56, *}$, J. A. Mcfayden ${ }^{78}$, G. Mchedlidze ${ }^{54}$, S. J. McMahon ${ }^{131}$, R. A. McPherson ${ }^{170, k}$, M. Medinnis ${ }^{42}$, S. Meehan ${ }^{146 a}$, S. Mehlhase ${ }^{100}$, A. Mehta ${ }^{74}$, K. Meier ${ }^{58 a}$, C. Meineck ${ }^{100}$, B. Meirose ${ }^{41}$, B. R. Mellado Garcia ${ }^{146 c}$, F. Meloni ${ }^{17}$, A. Mengarelli ${ }^{20 a, 20 b}$, S. Menke ${ }^{101}$, E. Meoni ${ }^{162}$, K. M. Mercurio ${ }^{57}$, S. Mergelmeyer ${ }^{21}$, P. Mermod ${ }^{49}$, L. Merola ${ }^{104 a, 104 b}$, C. Meroni ${ }^{91 a}$, F. S. Merritt ${ }^{31}$, A. Messina ${ }^{133 a, 133 a}$, J. Metcalfe ${ }^{25}$, A. S. Mete ${ }^{164}$, C. Meyer ${ }^{83}$, C. Meyer ${ }^{122}$ ， J-P. Meyer ${ }^{137}$, J. Meyer ${ }^{107}$ ， R. P. Middleton ${ }^{131}$, S. Miglioranzi ${ }^{165 a, 165 c}$, L. Mijović21, G. Mikenberg ${ }^{173}$, M. Mikestikova ${ }^{127}$, M. Mikuž $\check{z}^{75}$, M. Milesi ${ }^{88}$, A. Milic ${ }^{30}$, D. W. Miller ${ }^{31}$, C. Mills ${ }^{46}$, A. Milov ${ }^{173}$, D. A. Milstead ${ }^{147 a, 147 b}$, A. A. Minaenko ${ }^{130}$, Y. Minami ${ }^{156}$, I. A. Minashvili65, A. I. Mincer ${ }^{110}$, B. Mindur ${ }^{38 a}$, M. Mineev ${ }^{65}$, Y. Ming ${ }^{174}$, L. M. Mir ${ }^{12}$, T. Mitani ${ }^{172}$, J. Mitrevski ${ }^{100}$, V. A. Mitsou ${ }^{168}$, A. Miucci ${ }^{49}$, P. S. Miyagawa ${ }^{140}$, J. U. Mjörnmark ${ }^{81}$, T. Moa ${ }^{147 a, 147 b}$, K. Mochizuki ${ }^{85}$, S. Mohapatra ${ }^{35}$, W. Mohr ${ }^{48}$, S. Molander ${ }^{14 a, 147 b}$, R. Moles-Valls ${ }^{168}$, K. Mönig ${ }^{42}$, C. Monini ${ }^{55}$, J. Monk ${ }^{36}$, E. Monnier ${ }^{85}$, J. Montejo Berlingen ${ }^{12}$, F. Monticelli ${ }^{71}$, S. Monzani ${ }^{133 a, 133 b}$, R. W. Moore ${ }^{3}$, N. Morange ${ }^{117}$, D. Moreno ${ }^{163}$, M. Moreno Llácer ${ }^{54}$, P. Morettini ${ }^{50 a}$, M. Morgenstern ${ }^{44}$, M. Morii ${ }^{57}$, V. Morisbak ${ }^{119}$, S. Moritz ${ }^{83}$, A. K. Morley ${ }^{148}$, G. Mornacchi ${ }^{30}$, J. D. Morris ${ }^{76}$, S. S. Mortensen ${ }^{36}$, A. Morton ${ }^{53}$, L. Morvaj ${ }^{103}$, M. Mosidze ${ }^{51 b}$, J. Moss ${ }^{111}$, K. Motohashi ${ }^{158}$, R. Mount ${ }^{144}$, E. Mountricha ${ }^{25}$, S. V. Mouraviev ${ }^{96, *}$, E. J. W. Moyse ${ }^{86}$, S. Muanza ${ }^{85}$, R. D. Mudd ${ }^{18}$, F. Mueller ${ }^{101}$, J. Mueller ${ }^{125}$, K. Mueller ${ }^{21}$, R. S. P. Mueller ${ }^{100}$, T. Mueller ${ }^{28}$, D. Muenstermann ${ }^{49}$, P. Mullen ${ }^{53}$, Y. Munwes ${ }^{154}$, J. A. Murillo Quijada ${ }^{18}$, W. J. Murray ${ }^{171,131}$, H. Musheghyan ${ }^{54}$, E. Musto ${ }^{153}$, A. G. Myagkov ${ }^{130, a a}$, M. Myska ${ }^{128}$, O. Nackenhorst ${ }^{54}$, J. Nadal ${ }^{54}$, K. Nagai ${ }^{120}$, R. Nagai ${ }^{158}$, Y. Nagai ${ }^{85}$, K. Nagano ${ }^{66}$, A. Nagarkar ${ }^{111}$, Y. Nagasaka ${ }^{59}$, K. Nagata ${ }^{161}$, M. Nagel ${ }^{101}$, E. Nagy ${ }^{85}$, A. M. Nairz ${ }^{30}$, Y. Nakahama ${ }^{30}$, K. Nakamura ${ }^{66}$, T. Nakamura ${ }^{156}$, I. Nakano ${ }^{112}$, H. Namasivayam ${ }^{41}$, R. F. Naranjo Garcia ${ }^{42}$, R. Narayan ${ }^{58 b}$, T. Naumann ${ }^{42}$, G. Navarro ${ }^{163}$, R. Nayyar ${ }^{7}$, H. A. Neal ${ }^{89}$, P. Yu. Nechaeva ${ }^{96}$, T. J. Neep ${ }^{84}$, P. D. Nef ${ }^{144}$, A. Negri ${ }^{121 a, 121 b}$, M. Negrini ${ }^{20 a}$, S. Nektarijevic ${ }^{106}$, C. Nellist ${ }^{117}$, A. Nelson ${ }^{164}$, S. Nemecek ${ }^{127}$, P. Nemethy ${ }^{110}$, A. A. Nepomuceno ${ }^{24 a}$, M. Nessi ${ }^{30, a b}$, M. S. Neubauer ${ }^{166}$, M. Neumann ${ }^{176}$, R. M. Neves ${ }^{110}$, P. Nevski ${ }^{25}$, P. R. Newman ${ }^{18}$, D. H. Nguyen ${ }^{6}$, R. B. Nickerson ${ }^{120}$,

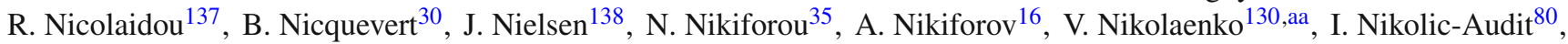
K. Nikolopoulos ${ }^{18}$, J. K. Nilsen ${ }^{119}$, P. Nilsson ${ }^{25}$, Y. Ninomiya ${ }^{156}$, A. Nisati ${ }^{133 a}$, R. Nisius ${ }^{101}$, T. Nobe ${ }^{158}$, M. Nomachi ${ }^{118}$, I. Nomidis ${ }^{29}$, T. Nooney ${ }^{76}$, S. Norberg ${ }^{113}$, M. Nordberg ${ }^{30}$, O. Novgorodova ${ }^{44}$, S. Nowak ${ }^{101}$, M. Nozaki ${ }^{66}$, L. Nozka ${ }^{115}$,

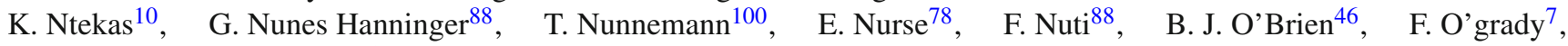
D. C. O’Neil ${ }^{143}$, V. O'Shea ${ }^{53}$, F. G. Oakham ${ }^{29, \mathrm{~d}}$, H. Oberlack ${ }^{101}$, T. Obermann ${ }^{21}$, J. Ocariz ${ }^{80}$, A. Ochi ${ }^{67}$, I. Ochoa ${ }^{78}$, J. P. Ochoa-Ricoux ${ }^{32 a}$, S. Oda ${ }^{70}$, S. Odaka ${ }^{66}$, H. Ogren ${ }^{61}$, A. $\mathrm{Oh}^{84}$, S. H. Oh${ }^{45}$, C. C. Ohm ${ }^{15}$, H. Ohman ${ }^{167}$, H. Oide ${ }^{30}$, W. Okamura ${ }^{18}$, H. Okawa ${ }^{161}$, Y. Okumura ${ }^{31}$, T. Okuyama ${ }^{156}$, A. Olariu ${ }^{26 a}$, S. A. Olivares Pino ${ }^{46}$, D. Oliveira Damazio ${ }^{25}$, E. Oliver Garcia ${ }^{168}$, A. Olszewski ${ }^{39}$, J. Olszowska ${ }^{39}$, A. Onofre ${ }^{126 a, 126 e}$, P. U. E. Onyisi ${ }^{31, q}$, C. J. Oram ${ }^{160 a}$, M. J. Oreglia ${ }^{31}$, Y. Oren ${ }^{154}$ ，D. Orestano ${ }^{135 a, 135 b}$ ，N. Orlando ${ }^{155}$, C. Oropeza Barrera ${ }^{53}$ ， R. S. Orr ${ }^{159}$ ，B. Osculati ${ }^{50 a, 50 b}$ ， R. Ospanov ${ }^{84}$, G. Otero y Garzon ${ }^{27}$, H. Otono ${ }^{70}$, M. Ouchrif ${ }^{136 d}$, E. A. Ouellette ${ }^{170}$, F. Ould-Saada ${ }^{119}$, A. Ouraou ${ }^{137}$, K. P. Oussoren ${ }^{107}$, Q. Ouyang ${ }^{33 a}$, A. Ovcharova ${ }^{15}$, M. Owen ${ }^{53}$, R. E. Owen ${ }^{18}$, V. E. Ozcan ${ }^{19 a}$, N. Ozturk ${ }^{8}$, K. Pachal ${ }^{120}$, A. Pacheco Pages ${ }^{12}$, C. Padilla Aranda ${ }^{12}$, M. Pagáčová48 ${ }^{48}$, S. Pagan Griso ${ }^{15}$, E. Paganis ${ }^{140}$, C. Pahl ${ }^{101}$, F. Paige ${ }^{25}$, P. Pais ${ }^{86}$, K. Pajchel ${ }^{119}$, G. Palacino ${ }^{160 b}$, S. Palestini ${ }^{30}$, M. Palka ${ }^{38 b}$, D. Pallin ${ }^{34}$, A. Palma ${ }^{126 a, 126 b}, \quad$ Y. B. Pan ${ }^{174}$, E. Panagiotopoulou ${ }^{10}$, 


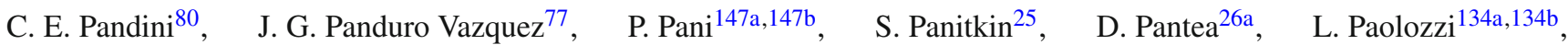
Th. D. Papadopoulou ${ }^{10}$, K. Papageorgiou ${ }^{155}$, A. Paramonov ${ }^{6}$, D. Paredes Hernandez ${ }^{155}$, M. A. Parker ${ }^{28}$, K. A. Parker ${ }^{140}$, F. Parodi ${ }^{50,50 b}$, J. A. Parsons ${ }^{35}$, U. Parzefall ${ }^{48}$, E. Pasqualucci ${ }^{133 a}$, S. Passaggio ${ }^{50 a}$, F. Pastore ${ }^{135 a, 135 b, *}$, Fr. Pastore ${ }^{77}$, G. Pásztor ${ }^{29}$ ， S. Pataraia ${ }^{176}$ ， N. D. Patel ${ }^{151}$ ， J. R. Pater ${ }^{84}$ ， T. Pauly ${ }^{30}$ ， J. Pearce ${ }^{170}$ ， B. Pearson ${ }^{113}$ ， L. E. Pedersen ${ }^{36}$, M. Pedersen ${ }^{119}$, S. Pedraza Lopez ${ }^{168}$, R. Pedro ${ }^{126 a, 126 b}$, S. V. Peleganchuk ${ }^{109}$, D. Pelikan ${ }^{167}$, H. Peng ${ }^{33 b}$, B. Penning ${ }^{31}$, J. Penwell ${ }^{61}$ ， D. V. Perepelitsa ${ }^{25}$, E. Perez Codina ${ }^{160 a}$, M. T. Pérez García-Estañ ${ }^{168}$, L. Perini ${ }^{91 a, 91 b}$, H. Pernegger ${ }^{30}$, S. Perrella ${ }^{104 a, 104 b}$, R. Peschke ${ }^{42}$, V. D. Peshekhonov ${ }^{65}$, K. Peters ${ }^{30}$, R. F. Y. Peters ${ }^{84}$, B. A. Petersen ${ }^{30}$, T. C. Petersen ${ }^{36}$, E. Petit ${ }^{42}$, A. Petridis ${ }^{147 a, 147 b}$, C. Petridou ${ }^{155}$, E. Petrolo ${ }^{133 a}$, F. Petrucci135a,135b , N. E. Pettersson ${ }^{158}$, R. Pezoa ${ }^{32 b}$, P. W. Phillips ${ }^{131}$, G. Piacquadio ${ }^{144}$, E. Pianori ${ }^{171}$, A. Picazio ${ }^{49}$, E. Piccaro ${ }^{76}$, M. Piccinini ${ }^{20 a, 20 b}$, M. A. Pickering ${ }^{120}$, R. Piegaia $^{27}$, D. T. Pignotti111, J. E. Pilcher ${ }^{31}$, A. D. Pilkington ${ }^{84}$, J. Pina ${ }^{126 a, 126 b, 126 d, ~ M . ~ P i n a m o n t i ~}{ }^{165 a, 165 c, a c}$, J. L. Pinfold ${ }^{3}$, A. Pingel ${ }^{36}$, B. Pinto ${ }^{126 a}$, S. Pires ${ }^{80}$, M. Pitt ${ }^{173}$, C. Pizio91a,91b, L. Plazak ${ }^{145 a}$ M. - A. Pleier ${ }^{25}$, V. Pleskot ${ }^{129}$, E. Plotnikova ${ }^{65}$, P. Plucinski ${ }^{147 a, 147 b}$, D. Pluth ${ }^{64}$, R. Poettgen ${ }^{83}$, L. Poggioli ${ }^{117}$, D. Pohl ${ }^{21}$, G. Polesello ${ }^{121 a}$, A. Policicchio ${ }^{37 a, 37 b}$, R. Polifka ${ }^{159}$, A. Polini ${ }^{20 a}$, C. S. Pollard ${ }^{53}$, V. Polychronakos ${ }^{25}$, K. Pommès ${ }^{30}$, L. Pontecorvo ${ }^{133 a}$, B. G. Pope ${ }^{90}$, G. A. Popeneciu ${ }^{26 b}$, D. S. Popovic ${ }^{13}$, A. Poppleton ${ }^{30}$, S. Pospisil ${ }^{128}$, K. Potamianos ${ }^{15}$, I. N. Potrap ${ }^{65}$, C. J. Potter ${ }^{150}$, C. T. Potter ${ }^{116}$, G. Poulard ${ }^{30}$, J. Poveda ${ }^{30}$, V. Pozdnyakov ${ }^{65}$, P. Pralavorio ${ }^{85}$, A. Pranko ${ }^{15}$, S. Prasad ${ }^{30}$, S. Prell ${ }^{64}$, D. Price ${ }^{84}$, J. Price ${ }^{74}$, L. E. Price ${ }^{6}$, M. Primavera ${ }^{73 a}$, S. Prince ${ }^{87}$, M. Proissl ${ }^{46}$, K. Prokofiev ${ }^{60 c}$, F. Prokoshin ${ }^{32 b}$, E. Protopapadaki ${ }^{137}$, S. Protopopescu ${ }^{25}$, J. Proudfoot ${ }^{6}$, M. Przybycien ${ }^{38 a}$, E. Ptacek ${ }^{116}$, D. Puddu ${ }^{135 a, 135 b}$, E. Pueschel ${ }^{86}$, D. Puldon ${ }^{149}$, M. Purohit ${ }^{25}$,ad , P. Puzo ${ }^{117}$, J. Qian ${ }^{89}$, G. Qin ${ }^{53}$, Y. Qin ${ }^{84}$, A. Quadt ${ }^{54}$, D. R. Quarrie ${ }^{15}$, W. B. Quayle ${ }^{165 a, 165 b}$, M. Queitsch-Maitland ${ }^{84}$, D. Quilty ${ }^{53}$, S. Raddum ${ }^{119}$, V. Radeka ${ }^{25}$, V. Radescu ${ }^{42}$, S. K. Radhakrishnan ${ }^{149}$, P. Radloff ${ }^{116}$, P. $\operatorname{Rados}^{88}$, F. Ragusa ${ }^{91 a}$,91b, G. Rahal ${ }^{179}$, S. Rajagopalan ${ }^{25}$, M. Rammensee ${ }^{30}$, C. Rangel-Smith ${ }^{167}$, F. Rauscher ${ }^{100}$, S. $\operatorname{Rave}^{83}$, T. Ravenscroft ${ }^{53}$, M. Raymond ${ }^{30}$, A. L. $\operatorname{Read}^{119}$, N. P. Readioff ${ }^{74}$, D. M. Rebuzzi ${ }^{121 a, 121 b}$, A. Redelbach ${ }^{175}$, G. Redlinger ${ }^{25}$, R. Reece ${ }^{138}$, K. Reeves ${ }^{41}$, L. Rehnisch ${ }^{16}$, H. Reisin ${ }^{27}$, M. Relich ${ }^{164}$, C. Rembser ${ }^{30}, \quad H$. Ren $^{33 a}$, A. Renaud ${ }^{117}$, M. Rescigno ${ }^{133 a}$, S. Resconi ${ }^{91 a}$ ， O. L. Rezanova ${ }^{109, c}$ ， P. Reznicek ${ }^{129}$ ， R. Rezvani ${ }^{95}$, R. Richter ${ }^{101}$, S. Richter ${ }^{78}$, E. Richter-Was ${ }^{38 b}$, O. Ricken ${ }^{21}$, M. Ridel ${ }^{80}$, P. Rieck ${ }^{16}$, C. J. Riegel ${ }^{176}$, J. Rieger ${ }^{54}$, M. Rijssenbeek ${ }^{149}$, A. Rimoldi121a,121b, L. Rinaldi ${ }^{20 a}$, B. Ristic ${ }^{49}$, E. Ritsch ${ }^{62}$, I. Riu ${ }^{12}$, F. Rizatdinova ${ }^{114}$, E. Rizvi ${ }^{76}$, S. H. Robertson ${ }^{87, k}$, A. Robichaud-Veronneau ${ }^{87}$, D. Robinson ${ }^{28}$, J. E. M. Robinson ${ }^{84}$, A. Robson ${ }^{53}$, C. Roda ${ }^{124 a, 124 b}$, S. Roe ${ }^{30}$, O. Røhne ${ }^{119}$, S. Rolli ${ }^{162}$, A. Romaniouk ${ }^{98}$, M. Romano ${ }^{20 \mathrm{a}, 20 \mathrm{~b}}$, S. M. Romano Saez ${ }^{34}$, E. Romero Adam ${ }^{168}$, N. Rompotis ${ }^{139}$, M. Ronzani ${ }^{48}$, L. $\operatorname{Roos}^{80}$, E. Ros ${ }^{168}$, S. Rosati ${ }^{133 a}$, K. Rosbach ${ }^{48}$, P. Rose ${ }^{138}$, P. L. Rosendahl ${ }^{14}$, O. Rosenthal ${ }^{142}$, V. Rossetti ${ }^{147 a, 147 b}$, E. Rossi ${ }^{104 a, 104 b}$, L. P. Rossi ${ }^{50 a}$, R. Rosten ${ }^{139}$, M. Rotaru ${ }^{26 a}$, I. Roth ${ }^{173}$, J. Rothberg ${ }^{139}$, D. Rousseau ${ }^{117}$, C. R. Royon ${ }^{137}$, A. Rozanov ${ }^{85}$, Y. Rozen ${ }^{153}$, X. Ruan ${ }^{146 c}$, F. Rubbo ${ }^{144}$, I. Rubinskiy ${ }^{42}$, V. I. Rud ${ }^{99}$, C. Rudolph ${ }^{44}$, M. S. Rudolph ${ }^{159}$, F. Rühr ${ }^{48}$, A. Ruiz-Martinez ${ }^{30}$, Z. Rurikova ${ }^{48}$, N. A. Rusakovich ${ }^{65}$, A. Ruschke ${ }^{100}$, H. L. Russel1 ${ }^{139}$, J. P. Rutherfoord ${ }^{7}$, N. Ruthmann ${ }^{48}$, Y. F. Ryabov ${ }^{123}$, M. Rybar ${ }^{129}$, G. Rybkin ${ }^{117}$, N. C. Ryder ${ }^{120}$, A. F. Saavedra ${ }^{151}$, G. Sabato ${ }^{107}$, S. Sacerdoti ${ }^{27}$, A. Saddique ${ }^{3}$, H. F-W. Sadrozinski ${ }^{138}$, R. Sadykov ${ }^{65}$, F. Safai Tehrani ${ }^{133 a}$, M. Saimpert ${ }^{137}$, H. Sakamoto ${ }^{156}$, Y. Sakurai ${ }^{172}$, G. Salamanna ${ }^{135 a, 135 b}$, A. Salamon ${ }^{134 a}$, M. Saleem ${ }^{113}$, D. Salek ${ }^{107}$, P. H. Sales De Bruin ${ }^{139}$, D. Salihagic ${ }^{101}$, A. Salnikov ${ }^{144}$, J. Salt ${ }^{168}$, D. Salvatore ${ }^{37 a, 37 b}$, F. Salvatore ${ }^{150}$, A. Salvucci ${ }^{106}, \quad$ A. Salzburger ${ }^{30}$, D. Sampsonidis ${ }^{155}$, A. Sanchez 104a,104b, J. Sánchez ${ }^{168}$, V. Sanchez Martinez ${ }^{168}$, H. Sandaker ${ }^{14}$, R. L. Sandbach ${ }^{76}$, H. G. Sander ${ }^{83}$, M. P. Sanders ${ }^{100}$, M. Sandhoff ${ }^{176}$, C. Sandoval ${ }^{163}$, R. Sandstroem ${ }^{101}$, D. P. C. Sankey ${ }^{131}$, M. Sannino ${ }^{50 a, 50 b}$, A. Sansoni ${ }^{47}$, C. Santoni ${ }^{34}$, R. Santonico ${ }^{134 a, 134 b}$, H. Santos ${ }^{126 a}$, I. Santoyo Castillo ${ }^{150}$, K. Sapp ${ }^{125}$, A. Sapronov ${ }^{65}$, J. G. Saraiva ${ }^{126 a, 126 d}$, B. Sarrazin ${ }^{21}$, O. Sasaki ${ }^{66}$, Y. Sasaki ${ }^{156}$, K. Sato ${ }^{161}$, G. Sauvage ${ }^{5, *}$, E. Sauvan ${ }^{5}$, G. Savage ${ }^{77}$, P. Savard ${ }^{159, d}$, C. Sawyer ${ }^{120}$, L. Sawyer ${ }^{79, n}$, J. Saxon ${ }^{31}$, C. Sbarra ${ }^{20 a}$, A. Sbrizzi ${ }^{20 a, 20 b}$, T. Scanlon ${ }^{78}$, D. A. Scannicchio ${ }^{164}$, M. Scarcella ${ }^{151}$, V. Scarfone ${ }^{37 a, 37 b}$, J. Schaarschmidt ${ }^{173}$, P. Schacht ${ }^{101}$, D. Schaefer ${ }^{30}$, R. Schaefer ${ }^{42}$, J. Schaeffer ${ }^{83}$, S. Schaepe ${ }^{21}$ ， S. Schaetzel ${ }^{58 b}$ ，U. Schäfer ${ }^{83}$ ，A. C. Schaffer ${ }^{117}$ ， D. Schaile ${ }^{100 ， \quad R . ~ D . ~ S c h a m b e r g e r ~}{ }^{149}$ ， V. Scharf ${ }^{58 a}$, V. A. Schegelsky ${ }^{123}$, D. Scheirich ${ }^{129}$, M. Schernau ${ }^{164}$, C. Schiavi ${ }^{50 a, 50 b}$, C. Schillo ${ }^{48}$, M. Schioppa ${ }^{37 a, 37 b}$, S. Schlenker ${ }^{30}$, E. Schmidt ${ }^{48}$, K. Schmieden ${ }^{30}$, C. Schmitt ${ }^{83}$, S. Schmitt ${ }^{58 b}$, S. Schmitt ${ }^{42}$, B. Schneider ${ }^{160 a}$, Y. J. Schnellbach ${ }^{74}$,

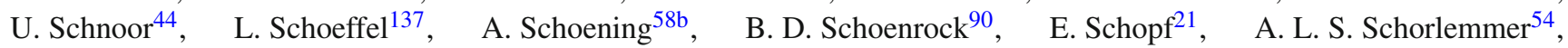
M. Schott ${ }^{83}$, D. Schouten ${ }^{160 a}$, J. Schovancova ${ }^{8}, \quad$ S. Schramm ${ }^{159}$, M. Schreyer ${ }^{175}, \quad$ C. Schroeder ${ }^{83}, \quad$ N. Schuh ${ }^{83}$, M. J. Schultens ${ }^{21}$, H.-C. Schultz-Coulon ${ }^{58 a}, \quad$ H. Schulz ${ }^{16}, \quad$ M. Schumacher ${ }^{48}$, B. A. Schumm ${ }^{138}, \quad$ Ph. Schune ${ }^{137}$, C. Schwanenberger ${ }^{84}$, A. Schwartzman ${ }^{144}$, T. A. Schwarz ${ }^{89}$, Ph. Schwegler ${ }^{101}$, Ph. Schwemling ${ }^{137}$, R. Schwienhorst ${ }^{90}$, J. Schwindling ${ }^{137}$, T. Schwindt ${ }^{21}$, M. Schwoerer ${ }^{5}$, F. G. Sciacca ${ }^{17}$, E. Scifo ${ }^{117}$, G. Sciolla ${ }^{23}$, F. Scuri ${ }^{124 a, 124 b}$, F. Scutti ${ }^{21}$, J. Searcy ${ }^{89}$ ， G. Sedov ${ }^{42}$, E. Sedykh ${ }^{123}$, P. Seema ${ }^{21}$, S. C. Seidel ${ }^{105}$, A. Seiden ${ }^{138}$, F. Seifert ${ }^{128}$, J. M. Seixas ${ }^{24 a}$, G. Sekhniaidze ${ }^{104 a}$ ， S. J. Sekula ${ }^{40}$ ， K. E. Selbach ${ }^{46}$ ， D. M. Seliverstov ${ }^{123, *}$ ， N. Semprini-Cesari20a,20b， C. Serfon ${ }^{30}$, L. Serin ${ }^{117}$, L. Serkin ${ }^{165 a, 165 b}$, T. Serre ${ }^{85}$, R. Seuster ${ }^{160 a}$, H. Severini ${ }^{113}$, T. Sfiligoj ${ }^{75}$, F. Sforza ${ }^{101}$, A. Sfyrla ${ }^{30}$, E. Shabalina ${ }^{54}$, M. Shamim ${ }^{116}$, L. Y. Shan ${ }^{33 a}$, R. Shang ${ }^{166}$, J. T. Shank ${ }^{22}$, M. Shapiro ${ }^{15}$, P. B. Shatalov ${ }^{97}$, K. Shaw ${ }^{165 a, 165 b}$, 
A. Shcherbakova ${ }^{147 a, 147 b}$, C. Y. Shehu ${ }^{150}$, P. Sherwood ${ }^{78}$, L. Shi ${ }^{152, a e}$, S. Shimizu ${ }^{67}$, C. O. Shimmin ${ }^{164}$, M. Shimojima ${ }^{102}$, M. Shiyakova ${ }^{65}$, A. Shmeleva ${ }^{96}$, D. Shoaleh Saadi ${ }^{95}$, M. J. Shochet ${ }^{31}$, S. Shojaii ${ }^{91 a, 91 b}$, S. Shrestha ${ }^{111}$, E. Shulga ${ }^{98}$, M. A. Shupe ${ }^{7}$, S. Shushkevich ${ }^{42}$, P. Sicho ${ }^{127}$, O. Sidiropoulou ${ }^{175}$, D. Sidorov ${ }^{114}$, A. Sidoti ${ }^{20,20 b}$, F. Siegert $^{44}$, Dj. Sijacki ${ }^{13}$, J. Silva ${ }^{126 a, 126 d}$, Y. Silver ${ }^{154}$ ，S. B. Silverstein ${ }^{147 a}$ ，V. Simak ${ }^{128}$ Ｏ. Simard ${ }^{5}$ ，Lj. Simic ${ }^{13}$ ，S. Simion ${ }^{117}$, E. Simioni ${ }^{83}$, B. Simmons ${ }^{78}$, D. Simon ${ }^{34}$, R. Simoniello ${ }^{91 a, 91 b}$, P. Sinervo ${ }^{159}$ ， N. B. Sinev ${ }^{116}$, G. Siragusa ${ }^{175}$, A. N. Sisakyan ${ }^{65, *}$, S. Yu. Sivoklokov ${ }^{99}$ ， J. Sjölin ${ }^{147 a, 147 b}$ ， T. B. Sjursen ${ }^{14}$, M. B. Skinner ${ }^{72}$, H. P. Skottowe ${ }^{57}$, P. Skubic ${ }^{113}$, M. Slater ${ }^{18}$, $\begin{array}{lll}\text { T. Slavicek } & 128\end{array}$ M. Slawinska ${ }^{107}$ ， K. Sliwa ${ }^{162}$, V. Smakhtin ${ }^{173}$ ， B. H. Smart ${ }^{46}$ ， L. Smestad ${ }^{14}$, S. Yu. Smirnov ${ }^{98}$, Y. Smirnov ${ }^{98}$, L. N. Smirnova ${ }^{99}$,af , O. Smirnova ${ }^{81}$, M. N. K. Smith ${ }^{35}$, M. Smizanska ${ }^{72}$, K. Smolek ${ }^{128}$, A. A. Snesarev ${ }^{96}$, G. Snidero ${ }^{76}$, S. Snyder ${ }^{25}$ ， R. Sobie ${ }^{170, k}$, F. Socher ${ }^{44}$, A. Soffer ${ }^{154}$ ， D. A. Soh ${ }^{152, \text { ae , C. A. Solans }}{ }^{30}$, M. Solar ${ }^{128}$, J. Solc ${ }^{128}$, E. Yu. Soldatov ${ }^{98}$, U. Soldevila ${ }^{168}$, A. A. Solodkov ${ }^{130}$, A. Soloshenko ${ }^{65}$, O. V. Solovyanov ${ }^{130}$, V. Solovyev ${ }^{123}$, P. Sommer ${ }^{48}$, H. Y. Song ${ }^{33 b}$, N. Soni ${ }^{1}$, A. Sood ${ }^{15}$, A. Sopczak ${ }^{128}$, B. Sopko ${ }^{128}$, V. Sopko ${ }^{128}$, V. Sorin ${ }^{12}$, D. Sosa ${ }^{58 b}$, M. Sosebee ${ }^{8}$, C. L. Sotiropoulou ${ }^{155}$, R. Soualah ${ }^{165 a, 165 c}$, P. Soueid ${ }^{95}$, A. M. Soukharev ${ }^{109, c}$, D. South ${ }^{42}$, S. Spagnolo ${ }^{73 a, 73 b}$, M. Spalla ${ }^{124 a, 124 b}$, F. Spanò ${ }^{77}$, W. R. Spearman ${ }^{57}$, F. Spettel ${ }^{101}$, R. Spighi ${ }^{20 a}$, G. Spigo ${ }^{30}$, L. A. Spiller ${ }^{88}$, M. Spousta ${ }^{129}$, T. Spreitzer ${ }^{159}$, R. D. St. Denis ${ }^{53, *}$, S. Staerz ${ }^{44}$, J. Stahlman ${ }^{122}$, R. Stamen ${ }^{58 a}$, S. Stamm ${ }^{16}$, E. Stanecka ${ }^{39}$, C. Stanescu ${ }^{135 a}$, M. Stanescu-Bellu ${ }^{42}$, M. M. Stanitzki ${ }^{42}$, S. Stapnes ${ }^{119}$, E. A. Starchenko ${ }^{130}$, J. Stark ${ }^{55}$, P. Staroba ${ }^{127}$, P. Starovoitov ${ }^{42}$, R. Staszewski ${ }^{39}$ ， P. Stavina ${ }^{145}$ a,* ，P. Steinberg ${ }^{25}$ ， B. Stelzer ${ }^{143}$ ， H. J. Stelzer ${ }^{30}$ ，O. Stelzer-Chilton ${ }^{160 a}$ ， H. Stenzel ${ }^{52}$, S. Stern ${ }^{101}$, G. A. Stewart ${ }^{53}$, J. A. Stillings ${ }^{21}$, M. C. Stockton ${ }^{87}$, M. Stoebe ${ }^{87}$, G. Stoicea ${ }^{26 a}$, P. Stolte ${ }^{54}$, S. Stonjek ${ }^{101}$, A. R. Stradling ${ }^{8}$, A. Straessner ${ }^{44}$, M. E. Stramaglia ${ }^{17}$, J. Strandberg ${ }^{148}$, S. Strandberg ${ }^{147 a, 147 b}$, A. Strandlie ${ }^{119}$, E. Strauss ${ }^{144}$, M. Strauss ${ }^{113}$, P. Strizenec ${ }^{145 b}$, R. Ströhmer ${ }^{175}$, D. M. Strom ${ }^{116}$, R. Stroynowski ${ }^{40}$, A. Strubig ${ }^{106}$, S. A. Stucci ${ }^{17}$, B. Stugu ${ }^{14}$, N. A. Styles ${ }^{42}$, D. Su ${ }^{144}$, J. Su ${ }^{125}$, R. Subramaniam ${ }^{79}$, A. Succurro ${ }^{12}$, Y. Sugaya ${ }^{118}$, C. Suhr ${ }^{108}$, M. Suk ${ }^{128}$, V. V. Sulin ${ }^{96}$, S. Sultansoy ${ }^{4 d}$, T. Sumida ${ }^{68}$, S. Sun ${ }^{57}$, X. Sun ${ }^{33 a}$, J. E. Sundermann ${ }^{48}$, K. Suruliz ${ }^{150}$, G. Susinno ${ }^{37 a, 37 b}$, M. R. Sutton ${ }^{150}$, S. Suzuki ${ }^{66}$, Y. Suzuki ${ }^{66}$, M. Svatos ${ }^{127}$, S. Swedish ${ }^{169}$, M. Swiatlowski ${ }^{144}$, I. Sykora ${ }^{145 a}$, T. Sykora ${ }^{129}$, D. Ta ${ }^{90}$,

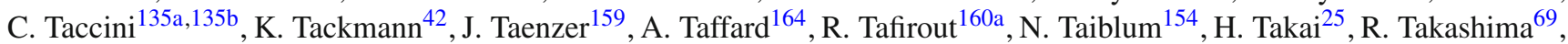
H. Takeda ${ }^{67}$, T. Takeshita' ${ }^{141}$, Y. Takubo ${ }^{66}$, M. Talby ${ }^{85}$, A. A. Talyshev ${ }^{109, c}$, J. Y. C. Tam ${ }^{175}$, K. G. Tan ${ }^{88}$, J. Tanaka ${ }^{156}$, R. Tanaka ${ }^{117}$, S. Tanaka ${ }^{132}$, S. Tanaka ${ }^{66}$, B. B. Tannenwald ${ }^{111}$, N. Tannoury ${ }^{21}$, S. Tapprogge ${ }^{83}$, S. Tarem ${ }^{153}$, F. Tarrade ${ }^{29}$, G. F. Tartarelli ${ }^{91 a}$, P. Tas ${ }^{129}$, M. Tasevsky ${ }^{127}$, T. Tashiro ${ }^{68}$, E. Tassi ${ }^{37 a, 37 b}$, A. Tavares Delgado ${ }^{126 a, 126 b}$, Y. Tayalati ${ }^{136 d}$, F. E. Taylor ${ }^{94}$, G. N. Taylor ${ }^{88}$, W. Taylor ${ }^{160 b}$, F. A. Teischinger ${ }^{30}$, M. Teixeira Dias Castanheira ${ }^{76}$, P. Teixeira-Dias ${ }^{77}$, K. K. Temming ${ }^{48}$, H. Ten Kate ${ }^{30}$, P. K. Teng ${ }^{152}$, J. J. Teoh ${ }^{118}$, F. Tepel ${ }^{176}$, S. Terada ${ }^{66}$, K. Terashi ${ }^{156}$, J. Terron ${ }^{82}$, S. Terzo ${ }^{101}$, M. Testa ${ }^{47}$, R. J. Teuscher ${ }^{159, k}$, J. Therhaag ${ }^{21}$, T. Theveneaux-Pelzer ${ }^{34}$, J. P. Thomas ${ }^{18}$, J. Thomas-Wilsker ${ }^{77}$, E. N. Thompson ${ }^{35}$, P. D. Thompson ${ }^{18}$, R. J. Thompson ${ }^{84}$, A. S. Thompson ${ }^{53}, \quad$ L. A. Thomsen ${ }^{36,}$ E. Thomson ${ }^{122}$, M. Thomson $^{28}$, R. P. Thun ${ }^{89, *}$, M. J. Tibbetts ${ }^{15}$, R. E. Ticse Torres ${ }^{85}$, V. O. Tikhomirov ${ }^{96, a g}$, Yu. A. Tikhonov ${ }^{109, c}$, S. Timoshenko ${ }^{98}$, E. Tiouchichine ${ }^{85}$, P. Tipton ${ }^{177}$, S. Tisserant ${ }^{85}$, T. Todorov ${ }^{5, *}$, S. Todorova-Nova ${ }^{129}$, J. Tojo ${ }^{70}$, S. Tokár ${ }^{145 a}$, K. Tokushuku ${ }^{66}, \quad$ K. Tollefson ${ }^{90}, \quad$ E. Tolley ${ }^{57}, \quad$ L. Tomlinson ${ }^{84}, \quad$ M. Tomoto ${ }^{103}, \quad$ L. Tompkins ${ }^{144, \text { ah }}$, K. Toms ${ }^{105}$, E. Torrence ${ }^{116}$, H. Torres ${ }^{143}$, E. Torró Pastor ${ }^{168}$, J. Toth ${ }^{85, a i}$, F. Touchard ${ }^{85}$, D. R. Tovey ${ }^{140}$, T. Trefzger ${ }^{175}$, L. Tremblet ${ }^{30}$, A. Tricoli $^{30}$, I. M. Trigger ${ }^{160 a}$, S. Trincaz-Duvoid ${ }^{80}$, M. F. Tripiana ${ }^{12}$, W. Trischuk ${ }^{159}$, B. Trocmé ${ }^{55}$, C. Troncon ${ }^{91 a}$, M. Trottier-McDonald ${ }^{15}$, M. Trovatelli ${ }^{135 a, 135 b}$, P. True ${ }^{90}$, M. Trzebinski ${ }^{39}$, A. Trzupek ${ }^{39}$, C. Tsarouchas $^{30}$, J. C-L. Tseng ${ }^{120}$, P. V. Tsiareshka ${ }^{92}$, D. Tsionou ${ }^{155}$, G. Tsipolitis ${ }^{10}$, N. Tsirintanis ${ }^{9}$, S. Tsiskaridze ${ }^{12}$, V. Tsiskaridze ${ }^{48}$, E. G. Tskhadadze ${ }^{51 \mathrm{a}}$, I. I. Tsukerman ${ }^{97}$, V. Tsulaia ${ }^{15}$, S. Tsuno ${ }^{66}$, D. Tsybychev ${ }^{149}$, A. Tudorache ${ }^{26 a}$, V. Tudorache ${ }^{26 a}$, A. N. Tuna ${ }^{122}$, S. A. Tupputi ${ }^{20 a, 20 b}$, S. Turchikhinn99,af, D. Turecek ${ }^{128}$, R. Turra ${ }^{91 a, 91 b}$, A. J. Turvey ${ }^{40}$, P. M. Tuts ${ }^{35}$,

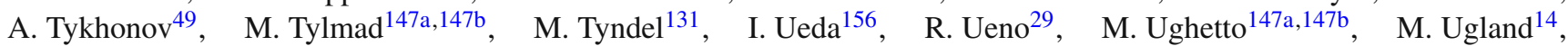
M. Uhlenbrock ${ }^{21}$, F. Ukegawa ${ }^{161}$, G. Unal $^{30}$, A. Undrus ${ }^{25}$, G. Unel ${ }^{164}$, F. C. Ungaro ${ }^{48}$, Y. Unno ${ }^{66}$, C. Unverdorben ${ }^{100}$, J. Urban ${ }^{145 b}$, P. Urquijo ${ }^{88}$, P. Urrejola ${ }^{83}$, G. Usai ${ }^{8}$, A. Usanova ${ }^{62}$, L. Vacavant ${ }^{85}$, V. Vacek ${ }^{128}$, B. Vachon ${ }^{87}$, C. Valderanis ${ }^{83}$, N. Valencic ${ }^{107}$, S. Valentinetti ${ }^{20 a}, 20 b$, A. Valero ${ }^{168}$, L. Valery ${ }^{12}$, S. Valkar ${ }^{129}$, E. Valladolid Gallego ${ }^{168}$, S. Vallecorsa ${ }^{49}$,

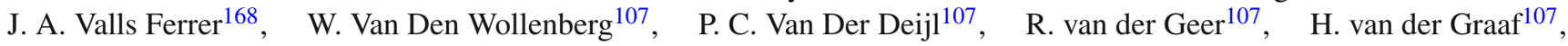
R. Van Der Leeuw ${ }^{107}$, N. van Eldik ${ }^{153}$, P. van Gemmeren ${ }^{6}$, J. Van Nieuwkoop ${ }^{143}$, I. van Vulpen ${ }^{107}$, M. C. van Woerden ${ }^{30}$, M. Vanadia ${ }^{133 a, 133 b}$, W. Vandelli ${ }^{30}$, R. Vanguri ${ }^{122}$, A. Vaniachine ${ }^{6}$, F. Vannucci ${ }^{80}$, G. Vardanyan ${ }^{178}$, R. Vari ${ }^{133 a}$, E. W. Varnes ${ }^{7}$, T. Varol ${ }^{40}$, D. Varouchas ${ }^{80}$, A. Vartapetian ${ }^{8}$, K. E. Varvell ${ }^{151}$, F. Vazeille ${ }^{34}$, T. Vazquez Schroeder ${ }^{87}$, J. Veatch ${ }^{7}$, F. Veloso ${ }^{126 a, 126 c}$, T. Velz ${ }^{21}$, S. Veneziano ${ }^{133 a}$, A. Ventura ${ }^{73 a, 73 b}$, D. Ventura ${ }^{86}$, M. Venturi ${ }^{170}$, N. Venturi ${ }^{159}$, A. Venturini ${ }^{23}$, V. Vercesi ${ }^{121 a}$, M. Verducci ${ }^{133 a, 133 b}$, W. Verkerke ${ }^{107}$, J. C. Vermeulen ${ }^{107}$, A. Vest ${ }^{44}$, M. C. Vetterli ${ }^{143, d}$, O. Viazlo ${ }^{81}$, I. Vichou ${ }^{166}$, T. Vickey ${ }^{140}$, O. E. Vickey Boeriu ${ }^{140}$ ， G. H. A. Viehhauser ${ }^{120}$ ， S. Viel ${ }^{15}$, R. Vigne ${ }^{30}$,

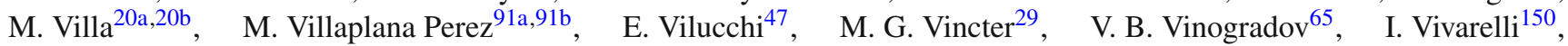
F. Vives Vaque ${ }^{3}$, S. Vlachos ${ }^{10}$, D. Vladoiu ${ }^{100}$, M. Vlasak ${ }^{128}$, M. Vogel ${ }^{32 a}$, P. Vokac ${ }^{128}$, G. Volpi ${ }^{124 a, 124 b}$, M. Volpi $^{88}$, H. von der Schmitt ${ }^{101}$, H. von Radziewski ${ }^{48}$, E. von Toerne ${ }^{21}$, V. Vorobel ${ }^{129}$, K. Vorobev ${ }^{98}$, M. Vos ${ }^{168}$, R. Voss ${ }^{30}$, 
J. H. Vossebeld ${ }^{74}$, N. Vranjes ${ }^{13}$, M. Vranjes Milosavljevic ${ }^{13}$, V. Vrba ${ }^{127}$, M. Vreeswijk ${ }^{107}$, R. Vuillermet ${ }^{30}$, I. Vukotic ${ }^{31}$, Z. Vykydal ${ }^{128}$, P. Wagner ${ }^{21}$, W. Wagner ${ }^{176}$, H. Wahlberg ${ }^{71}$, S. Wahrmund ${ }^{44}$, J. Wakabayashi ${ }^{103}$, J. Walder ${ }^{72}$, R. Walker ${ }^{100}$, W. Walkowiak ${ }^{142}$, C. Wang ${ }^{33 \mathrm{c}}$, F. Wang ${ }^{174}$, H. Wang ${ }^{15}$, H. Wang ${ }^{40}$, J. Wang ${ }^{42}$, J. Wang ${ }^{33 a}$, K. Wang ${ }^{87}$, R. Wang ${ }^{6}$, S. M. Wang ${ }^{152}$, T. Wang ${ }^{21}$, X. Wang ${ }^{177}$, C. Wanotayaroj ${ }^{116}$, A. Warburton ${ }^{87}$, C. P. Ward ${ }^{28}$, D. R. Wardrope ${ }^{78}$, M. Warsinsky ${ }^{48}$, A. Washbrook ${ }^{46}$, C. Wasicki ${ }^{42}$, P. M. Watkins ${ }^{18}$, A. T. Watson ${ }^{18}$, I. J. Watson ${ }^{151}$, M. F. Watson ${ }^{18}$, G. Watts ${ }^{139}$, S. Watts ${ }^{84}$, B. M. Waugh ${ }^{78}$, S. Webb ${ }^{84}$, M. S. Weber ${ }^{17}$, S. W. Weber ${ }^{175}$, J. S. Webster ${ }^{31}$, A. R. Weidberg ${ }^{120}$, B. Weinert ${ }^{61}$, J. Weingarten ${ }^{54}$, C. Weiser ${ }^{48}$, H. Weits ${ }^{107}$, P. S. Wells ${ }^{30}$, T. Wenaus ${ }^{25}$, T. Wengler ${ }^{30}$, S. Wenig ${ }^{30}$, N. Wermes ${ }^{21}$, M. Werner ${ }^{48}$, P. Werner ${ }^{30}$, M. Wessels ${ }^{58 a}$, J. Wetter ${ }^{162}$, K. Whalen ${ }^{29}$, A. M. Wharton ${ }^{72}$, A. White ${ }^{8}$, M. J. White ${ }^{1}$, R. White ${ }^{32 b}$, S. White ${ }^{124 a, 124 b}$, D. Whiteson ${ }^{164}$, F. J. Wickens ${ }^{131}$, W. Wiedenmann ${ }^{174}$, M. Wielers ${ }^{131}$, P. Wienemann ${ }^{21}$, C. Wiglesworth ${ }^{36}$, L. A. M. Wiik-Fuchs ${ }^{21}$, A. Wildauer ${ }^{101}$, H. G. Wilkens ${ }^{30}$, H. H. Williams ${ }^{122}$, S. Williams ${ }^{107}$, C. Willis ${ }^{90}$, S. Willocq ${ }^{86}$, A. Wilson ${ }^{89}$, J. A. Wilson ${ }^{18}$, I. Wingerter-Seez ${ }^{5}$, F. Winklmeier ${ }^{116}$, B. T. Winter ${ }^{21}$, M. Wittgen ${ }^{144}$, J. Wittkowski ${ }^{100}$, S. J. Wollstadt ${ }^{83}$, M. W. Wolter ${ }^{39}$, H. Wolters ${ }^{126 a, 126 c}$, B. K. Wosiek ${ }^{39}$, J. Wotschack ${ }^{30}$, M. J. Woudstra ${ }^{84}$, K. W. Wozniak ${ }^{39}$, M. Wu ${ }^{55}$, M. Wu ${ }^{31}$, S. L. Wu ${ }^{174}$, X. Wu ${ }^{49}$, Y. Wu ${ }^{89}$, T. R. Wyatt ${ }^{84}$, B. M. Wynne ${ }^{46}$, S. Xella ${ }^{36}$, D. Xu ${ }^{33 a}$, L. Xu ${ }^{33 b, a j}$, B. Yabsley ${ }^{151}$, S. Yacoob ${ }^{146 b, a k}$, R. Yakabe ${ }^{67}$, M. Yamada ${ }^{66}$, Y. Yamaguchi ${ }^{118}$, A. Yamamoto ${ }^{66}$, S. Yamamoto ${ }^{156}$, T. Yamanaka ${ }^{156}$, K. Yamauchi ${ }^{103}$, Y. Yamazaki ${ }^{67}$, Z. Yan ${ }^{22}$, H. Yang ${ }^{33 e}$, H. Yang ${ }^{174}$, Y. Yang ${ }^{152}$, L. Yao ${ }^{33 a}$, W-M. Yao ${ }^{15}$, Y. Yasu ${ }^{66}$, E. Yatsenko ${ }^{42}$, K. H. Yau Wong ${ }^{21}$, J. Ye ${ }^{40}$, S. Ye ${ }^{25}$, I. Yeletskikh ${ }^{65}$, A. L. Yen ${ }^{57}$, E. Yildirim ${ }^{42}$, K. Yorita ${ }^{172}$, R. Yoshida ${ }^{6}$, K. Yoshihara ${ }^{122}$, C. Young ${ }^{144}$, C. J. S. Young ${ }^{30}$, S. Youssef ${ }^{22}$, D. R. Yu ${ }^{15}$, J. Yu ${ }^{8}$, J. M. Yu ${ }^{89}$, J. Yu ${ }^{114}$, L. Yuan ${ }^{67}$, A. Yurkewicz ${ }^{108}$, I. Yusuff ${ }^{28, a l}$, B. Zabinski ${ }^{39}$, R. Zaidan ${ }^{63}$, A. M. Zaitsev ${ }^{130, a a,}$ J. Zalieckas ${ }^{14}$, A. Zaman ${ }^{149}$, S. Zambito ${ }^{23}$, L. Zanello133a,133b, D. Zanzi ${ }^{88}$, C. Zeitnitz ${ }^{176}$, M. Zeman ${ }^{128}$, A. Zemla ${ }^{38 a}$, K. Zengel ${ }^{23}$, O. Zenin ${ }^{130}$, T. Ženišs ${ }^{145 a}$, D. Zerwas ${ }^{117}$, D. Zhang ${ }^{89}$, F. Zhang ${ }^{174}$, J. Zhang ${ }^{6}$, L. Zhang ${ }^{48}$, R. Zhang ${ }^{33 b}$, X. Zhang ${ }^{33 d}$, Z. Zhang ${ }^{117}$, X. Zhao ${ }^{40}$, Y. Zhao ${ }^{33 d, 117}$, Z. Zhao ${ }^{33 b}$, A. Zhemchugov ${ }^{65}$, J. Zhong ${ }^{120}$, B. Zhou ${ }^{89}$, C. Zhou ${ }^{45}$, L. Zhou ${ }^{35}$, L. Zhou ${ }^{40}$, N. Zhou ${ }^{164}$, C. G. Zhu ${ }^{33 d}$, H. Zhu ${ }^{33 a}$, J. Zhu ${ }^{89}$, Y. Zhu ${ }^{33 b}$, X. Zhuang ${ }^{33 a}$, K. Zhukov ${ }^{96}$, A. Zibell ${ }^{175}$, D. Zieminska ${ }^{61}$, N. I. Zimine ${ }^{65}$, C. Zimmermann ${ }^{83}$, R. Zimmermann ${ }^{21}$, S. Zimmermann ${ }^{48}$, Z. Zinonos ${ }^{54}$, M. Zinser ${ }^{83}$, M. Ziolkowski ${ }^{142}$, L. Živković ${ }^{13}$, G. Zobernig ${ }^{174}$, A. Zoccoli ${ }^{20 a, 20 b}$, M. zur Nedden ${ }^{16}$, G. Zurzolo ${ }^{104 a, 104 b}$, L. Zwalinski ${ }^{30}$

${ }^{1}$ Department of Physics, University of Adelaide, Adelaide, Australia

${ }^{2}$ Physics Department, SUNY Albany, Albany, NY, USA

${ }^{3}$ Department of Physics, University of Alberta, Edmonton, AB, Canada

4 (a) Department of Physics, Ankara University, Ankara, Turkey; (b) Istanbul Aydin University, Istanbul, Turkey;

(c) Division of Physics, TOBB University of Economics and Technology, Ankara, Turkey

${ }^{5}$ LAPP, CNRS/IN2P3 and Université Savoie Mont Blanc, Annecy-le-Vieux, France

${ }^{6}$ High Energy Physics Division, Argonne National Laboratory, Argonne, IL, USA

${ }^{7}$ Department of Physics, University of Arizona, Tucson, AZ, USA

${ }^{8}$ Department of Physics, The University of Texas at Arlington, Arlington, TX, USA

${ }^{9}$ Physics Department, University of Athens, Athens, Greece

${ }^{10}$ Physics Department, National Technical University of Athens, Zografou, Greece

${ }^{11}$ Institute of Physics, Azerbaijan Academy of Sciences, Baku, Azerbaijan

12 Institut de Física d'Altes Energies and Departament de Física de la Universitat Autònoma de Barcelona, Barcelona, Spain

${ }^{13}$ Institute of Physics, University of Belgrade, Belgrade, Serbia

${ }^{14}$ Department for Physics and Technology, University of Bergen, Bergen, Norway

${ }^{15}$ Physics Division, Lawrence Berkeley National Laboratory and University of California, Berkeley, CA, USA

${ }^{16}$ Department of Physics, Humboldt University, Berlin, Germany

${ }^{17}$ Albert Einstein Center for Fundamental Physics and Laboratory for High Energy Physics, University of Bern, Bern, Switzerland

${ }^{18}$ School of Physics and Astronomy, University of Birmingham, Birmingham, UK

19 (a) Department of Physics, Bogazici University, Istanbul, Turkey; ${ }^{(b)}$ Department of Physics, Dogus University, Istanbul,

Turkey; (c) Department of Physics Engineering, Gaziantep University, Gaziantep, Turkey

20 (a) INFN Sezione di Bologna, Bologna, Italy; (b) Dipartimento di Fisica e Astronomia, Università di Bologna, Bologna, Italy

${ }^{21}$ Physikalisches Institut, University of Bonn, Bonn, Germany

${ }^{22}$ Department of Physics, Boston University, Boston, MA, USA

${ }^{23}$ Department of Physics, Brandeis University, Waltham, MA, USA 
24 (a) Universidade Federal do Rio De Janeiro COPPE/EE/IF, Rio de Janeiro, Brazil; (b) Electrical Circuits Department, Federal University of Juiz de Fora (UFJF), Juiz de Fora, Brazil; (c) Federal University of Sao Joao del Rei (UFSJ), Sao Joao del Rei, Brazil; ${ }^{(d)}$ Instituto de Fisica, Universidade de Sao Paulo, São Paulo, Brazil

${ }^{25}$ Physics Department, Brookhaven National Laboratory, Upton, NY, USA

26 (a) National Institute of Physics and Nuclear Engineering, Bucharest, Romania; (b) Physics Department, National Institute for Research and Development of Isotopic and Molecular Technologies, Cluj Napoca, Romania; (c) University Politehnica Bucharest, Bucharest, Romania; (d) West University in Timisoara, Timisoara, Romania

${ }^{27}$ Departamento de Física, Universidad de Buenos Aires, Buenos Aires, Argentina

${ }^{28}$ Cavendish Laboratory, University of Cambridge, Cambridge, UK

${ }^{29}$ Department of Physics, Carleton University, Ottawa, ON, Canada

${ }^{30}$ CERN, Geneva, Switzerland

${ }^{31}$ Enrico Fermi Institute, University of Chicago, Chicago, IL, USA

32 (a) Departamento de Física, Pontificia Universidad Católica de Chile, Santiago, Chile; (b) Departamento de Física, Universidad Técnica Federico Santa María, Valparaiso, Chile

33 (a) Institute of High Energy Physics, Chinese Academy of Sciences, Beijing, China; (b) Department of Modern Physics, University of Science and Technology of China, Anhui, China; ${ }^{(c)}$ Department of Physics, Nanjing University, Jiangsu, China; (d) School of Physics, Shandong University, Shandong, China; (e) Department of Physics and Astronomy, Shanghai Key Laboratory for Particle Physics and Cosmology, Shanghai Jiao Tong University, Shanghai, China;

(f) Physics Department, Tsinghua University, 100084 Beijing, China

${ }^{34}$ Laboratoire de Physique Corpusculaire, Clermont Université and Université Blaise Pascal and CNRS/IN2P3,

Clermont-Ferrand, France

${ }^{35}$ Nevis Laboratory, Columbia University, Irvington, NY, USA

${ }^{36}$ Niels Bohr Institute, University of Copenhagen, Copenhagen, Denmark

37 (a) INFN Gruppo Collegato di Cosenza, Laboratori Nazionali di Frascati, Frascati, Italy; (b) Dipartimento di Fisica, Università della Calabria, Rende, Italy

38 (a) Faculty of Physics and Applied Computer Science, AGH University of Science and Technology, Kraków, Poland;

(b) Marian Smoluchowski Institute of Physics, Jagiellonian University, Kraków, Poland

${ }^{39}$ Institute of Nuclear Physics, Polish Academy of Sciences, Kraków, Poland

${ }^{40}$ Physics Department, Southern Methodist University, Dallas, TX, USA

${ }^{41}$ Physics Department, University of Texas at Dallas, Richardson, TX, USA

42 DESY, Hamburg and Zeuthen, Germany

${ }^{43}$ Institut für Experimentelle Physik IV, Technische Universität Dortmund, Dortmund, Germany

${ }^{44}$ Institut für Kern- und Teilchenphysik, Technische Universität Dresden, Dresden, Germany

${ }^{45}$ Department of Physics, Duke University, Durham, NC, USA

46 SUPA-School of Physics and Astronomy, University of Edinburgh, Edinburgh, UK

${ }^{47}$ INFN Laboratori Nazionali di Frascati, Frascati, Italy

${ }^{48}$ Fakultät für Mathematik und Physik, Albert-Ludwigs-Universität, Freiburg, Germany

${ }^{49}$ Section de Physique, Université de Genève, Geneva, Switzerland

50 (a) INFN Sezione di Genova, Genova, Italy; (b) Dipartimento di Fisica, Università di Genova, Genova, Italy

51 (a) E. Andronikashvili Institute of Physics, Iv. Javakhishvili Tbilisi State University, Tbilisi, Georgia; (b) High Energy

Physics Institute, Tbilisi State University, Tbilisi, Georgia

52 II Physikalisches Institut, Justus-Liebig-Universität Giessen, Giessen, Germany

${ }^{53}$ SUPA-School of Physics and Astronomy, University of Glasgow, Glasgow, UK

54 II Physikalisches Institut, Georg-August-Universität, Göttingen, Germany

${ }^{55}$ Laboratoire de Physique Subatomique et de Cosmologie, Université Grenoble-Alpes, CNRS/IN2P3, Grenoble, France

${ }^{56}$ Department of Physics, Hampton University, Hampton, VA, USA

${ }^{57}$ Laboratory for Particle Physics and Cosmology, Harvard University, Cambridge, MA, USA

58 (a) Kirchhoff-Institut für Physik, Ruprecht-Karls-Universität Heidelberg, Heidelberg, Germany; ${ }^{(b)}$ Physikalisches Institut, Ruprecht-Karls-Universität Heidelberg, Heidelberg, Germany; ${ }^{(c)}$ ZITI Institut für technische Informatik, Ruprecht-Karls-Universität Heidelberg, Mannheim, Germany

${ }^{59}$ Faculty of Applied Information Science, Hiroshima Institute of Technology, Hiroshima, Japan 
60 (a) Department of Physics, The Chinese University of Hong Kong, Shatin, NT, Hong Kong; (b) Department of Physics, The University of Hong Kong, Pok Fu Lam, Hong Kong; (c) Department of Physics, The Hong Kong University of Science and Technology, Clear Water Bay, Kowloon, Hong Kong, China

${ }^{61}$ Department of Physics, Indiana University, Bloomington, IN, USA

${ }^{62}$ Institut für Astro- und Teilchenphysik, Leopold-Franzens-Universität, Innsbruck, Austria

${ }^{63}$ University of Iowa, Iowa City, IA, USA

${ }^{64}$ Department of Physics and Astronomy, Iowa State University, Ames, IA, USA

65 Joint Institute for Nuclear Research, JINR Dubna, Dubna, Russia

${ }^{66}$ KEK, High Energy Accelerator Research Organization, Tsukuba, Japan

${ }^{67}$ Graduate School of Science, Kobe University, Kobe, Japan

${ }^{68}$ Faculty of Science, Kyoto University, Kyoto, Japan

${ }^{69}$ Kyoto University of Education, Kyoto, Japan

70 Department of Physics, Kyushu University, Fukuoka, Japan

${ }^{71}$ Instituto de Física La Plata, Universidad Nacional de La Plata and CONICET, La Plata, Argentina

72 Physics Department, Lancaster University, Lancaster, UK

73 (a) INFN Sezione di Lecce, Lecce, Italy; ${ }^{\text {(b) }}$ Dipartimento di Matematica e Fisica, Università del Salento, Lecce, Italy

${ }^{74}$ Oliver Lodge Laboratory, University of Liverpool, Liverpool, UK

${ }^{75}$ Department of Physics, Jožef Stefan Institute and University of Ljubljana, Ljubljana, Slovenia

${ }^{76}$ School of Physics and Astronomy, Queen Mary University of London, London, UK

77 Department of Physics, Royal Holloway University of London, Surrey, UK

${ }^{78}$ Department of Physics and Astronomy, University College London, London, UK

${ }^{79}$ Louisiana Tech University, Ruston, LA, USA

${ }^{80}$ Laboratoire de Physique Nucléaire et de Hautes Energies, UPMC and Université Paris-Diderot and CNRS/IN2P3, Paris, France

${ }^{81}$ Fysiska institutionen, Lunds universitet, Lund, Sweden

${ }^{82}$ Departamento de Fisica Teorica C-15, Universidad Autonoma de Madrid, Madrid, Spain

${ }^{83}$ Institut für Physik, Universität Mainz, Mainz, Germany

${ }^{84}$ School of Physics and Astronomy, University of Manchester, Manchester, UK

${ }^{85}$ CPPM, Aix-Marseille Université and CNRS/IN2P3, Marseille, France

${ }^{86}$ Department of Physics, University of Massachusetts, Amherst, MA, USA

${ }^{87}$ Department of Physics, McGill University, Montreal, QC, Canada

${ }^{88}$ School of Physics, University of Melbourne, Melbourne, VIC, Australia

${ }^{89}$ Department of Physics, The University of Michigan, Ann Arbor, MI, USA

${ }^{90}$ Department of Physics and Astronomy, Michigan State University, East Lansing, MI, USA

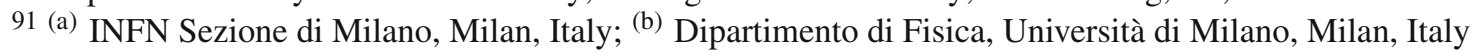

92 B.I. Stepanov Institute of Physics, National Academy of Sciences of Belarus, Minsk, Republic of Belarus

${ }^{93}$ National Scientific and Educational Centre for Particle and High Energy Physics, Minsk, Republic of Belarus

${ }^{94}$ Department of Physics, Massachusetts Institute of Technology, Cambridge, MA, USA

${ }^{95}$ Group of Particle Physics, University of Montreal, Montreal, QC, Canada

${ }^{96}$ P.N. Lebedev Institute of Physics, Academy of Sciences, Moscow, Russia

${ }^{97}$ Institute for Theoretical and Experimental Physics (ITEP), Moscow, Russia

98 National Research Nuclear University MEPhI, Moscow, Russia

${ }^{99}$ D.V. Skobeltsyn Institute of Nuclear Physics, M.V. Lomonosov Moscow State University, Moscow, Russia

${ }^{100}$ Fakultät für Physik, Ludwig-Maximilians-Universität München, Munich, Germany

${ }^{101}$ Max-Planck-Institut für Physik (Werner-Heisenberg-Institut), Munich, Germany

102 Nagasaki Institute of Applied Science, Nagasaki, Japan

${ }^{103}$ Graduate School of Science and Kobayashi-Maskawa Institute, Nagoya University, Nagoya, Japan

104 (a) INFN Sezione di Napoli, Naples, Italy; ${ }^{\text {(b) }}$ Dipartimento di Fisica, Università di Napoli, Naples, Italy

105 Department of Physics and Astronomy, University of New Mexico, Albuquerque, NM, USA

106 Institute for Mathematics, Astrophysics and Particle Physics, Radboud University Nijmegen/Nikhef, Nijmegen,

The Netherlands

${ }^{107}$ Nikhef National Institute for Subatomic Physics and University of Amsterdam, Amsterdam, The Netherlands

108 Department of Physics, Northern Illinois University, De Kalb, IL, USA 
109 Budker Institute of Nuclear Physics, SB RAS, Novosibirsk, Russia

${ }^{110}$ Department of Physics, New York University, New York, NY, USA

111 Ohio State University, Columbus, OH, USA

${ }^{112}$ Faculty of Science, Okayama University, Okayama, Japan

${ }^{113}$ Homer L. Dodge Department of Physics and Astronomy, University of Oklahoma, Norman, OK, USA

${ }^{114}$ Department of Physics, Oklahoma State University, Stillwater, OK, USA

115 Palacký University, RCPTM, Olomouc, Czech Republic

116 Center for High Energy Physics, University of Oregon, Eugene, OR, USA

${ }^{117}$ LAL, Université Paris-Sud and CNRS/IN2P3, Orsay, France

${ }^{118}$ Graduate School of Science, Osaka University, Osaka, Japan

119 Department of Physics, University of Oslo, Oslo, Norway

120 Department of Physics, Oxford University, Oxford, UK

121 (a) INFN Sezione di Pavia, Pavia, Italy; (b) Dipartimento di Fisica, Università di Pavia, Pavia, Italy

122 Department of Physics, University of Pennsylvania, Philadelphia, PA, USA

${ }^{123}$ Petersburg Nuclear Physics Institute, Gatchina, Russia

124 (a) INFN Sezione di Pisa, Pisa, Italy; ${ }^{(b)}$ Dipartimento di Fisica E. Fermi, Università di Pisa, Pisa, Italy

125 Department of Physics and Astronomy, University of Pittsburgh, Pittsburgh, PA, USA

126 (a) Laboratorio de Instrumentacao e Fisica Experimental de Particulas-LIP, Lisbon, Portugal; (b) Faculdade de Ciências, Universidade de Lisboa, Lisbon, Portugal; (c) Department of Physics, University of Coimbra, Coimbra, Portugal; (d) Centro de Física Nuclear da Universidade de Lisboa, Lisbon, Portugal; (e) Departamento de Fisica, Universidade do Minho, Braga, Portugal; (f) Departamento de Fisica Teorica y del Cosmos and CAFPE, Universidad de Granada, Granada, Spain; ${ }^{(g)}$ Dep Fisica and CEFITEC of Faculdade de Ciencias e Tecnologia, Universidade Nova de Lisboa, Caparica, Portugal

${ }^{127}$ Institute of Physics, Academy of Sciences of the Czech Republic, Prague, Czech Republic

${ }^{128}$ Czech Technical University in Prague, Prague, Czech Republic

${ }^{129}$ Faculty of Mathematics and Physics, Charles University in Prague, Prague, Czech Republic

${ }^{130}$ State Research Center Institute for High Energy Physics, Protvino, Russia

${ }^{131}$ Particle Physics Department, Rutherford Appleton Laboratory, Didcot, UK

${ }^{132}$ Ritsumeikan University, Kusatsu, Shiga, Japan

133 (a) INFN Sezione di Roma, Rome, Italy; (b) Dipartimento di Fisica, Sapienza Università di Roma, Rome, Italy

134 (a) INFN Sezione di Roma Tor Vergata, Rome, Italy; ${ }^{(b)}$ Dipartimento di Fisica, Università di Roma Tor Vergata, Rome, Italy

135 (a) INFN Sezione di Roma Tre, Rome, Italy; (b) Dipartimento di Matematica e Fisica, Università Roma Tre, Rome, Italy

136 (a) Faculté des Sciences Ain Chock, Réseau Universitaire de Physique des Hautes Energies-Université Hassan II, Casablanca, Morocco; ${ }^{(b)}$ Centre National de l'Energie des Sciences Techniques Nucleaires, Rabat, Morocco; ${ }^{(c)}$ Faculté des Sciences Semlalia, Université Cadi Ayyad, LPHEA-Marrakech, Marrakech, Morocco; (d) Faculté des Sciences, Université Mohamed Premier and LPTPM, Oujda, Morocco; ${ }^{(e)}$ Faculté des Sciences, Université Mohammed V-Agdal, Rabat, Morocco

${ }^{137}$ DSM/IRFU (Institut de Recherches sur les Lois Fondamentales de l'Univers), CEA Saclay (Commissariat à l'Energie Atomique et aux Energies Alternatives), Gif-sur-Yvette, France

${ }^{138}$ Santa Cruz Institute for Particle Physics, University of California Santa Cruz, Santa Cruz, CA, USA

${ }^{139}$ Department of Physics, University of Washington, Seattle, WA, USA

${ }^{140}$ Department of Physics and Astronomy, University of Sheffield, Sheffield, UK

${ }^{141}$ Department of Physics, Shinshu University, Nagano, Japan

${ }^{142}$ Fachbereich Physik, Universität Siegen, Siegen, Germany

${ }^{143}$ Department of Physics, Simon Fraser University, Burnaby, BC, Canada

${ }^{144}$ SLAC National Accelerator Laboratory, Stanford, CA, USA

145 (a) Faculty of Mathematics, Physics and Informatics, Comenius University, Bratislava, Slovak Republic; (b) Department of Subnuclear Physics, Institute of Experimental Physics of the Slovak Academy of Sciences, Kosice, Slovak Republic

146 (a) Department of Physics, University of Cape Town, Cape Town, South Africa; (b) Department of Physics, University of Johannesburg, Johannesburg, South Africa; ${ }^{\text {(c) }}$ School of Physics, University of the Witwatersrand, Johannesburg, South Africa

147 (a) Department of Physics, Stockholm University, Stockholm, Sweden; ${ }^{\text {(b) }}$ The Oskar Klein Centre, Stockholm, Sweden 
148 Physics Department, Royal Institute of Technology, Stockholm, Sweden

${ }^{149}$ Departments of Physics and Astronomy and Chemistry, Stony Brook University, Stony Brook, NY, USA

${ }^{150}$ Department of Physics and Astronomy, University of Sussex, Brighton, UK

${ }^{151}$ School of Physics, University of Sydney, Sydney, Australia

152 Institute of Physics, Academia Sinica, Taipei, Taiwan

${ }^{153}$ Department of Physics, Technion: Israel Institute of Technology, Haifa, Israel

154 Raymond and Beverly Sackler School of Physics and Astronomy, Tel Aviv University, Tel Aviv, Israel

${ }^{155}$ Department of Physics, Aristotle University of Thessaloniki, Thessaloniki, Greece

${ }^{156}$ International Center for Elementary Particle Physics and Department of Physics, The University of Tokyo, Tokyo, Japan

${ }^{157}$ Graduate School of Science and Technology, Tokyo Metropolitan University, Tokyo, Japan

${ }^{158}$ Department of Physics, Tokyo Institute of Technology, Tokyo, Japan

${ }^{159}$ Department of Physics, University of Toronto, Toronto, ON, Canada

160 (a) TRIUMF, Vancouver, BC, Canada; (b) Department of Physics and Astronomy, York University, Toronto, ON, Canada

${ }^{161}$ Faculty of Pure and Applied Sciences, University of Tsukuba, Tsukuba, Japan

162 Department of Physics and Astronomy, Tufts University, Medford, MA, USA

${ }^{163}$ Centro de Investigaciones, Universidad Antonio Narino, Bogota, Colombia

${ }^{164}$ Department of Physics and Astronomy, University of California Irvine, Irvine, CA, USA

165 (a) INFN Gruppo Collegato di Udine, Sezione di Trieste, Udine, Italy; ${ }^{(b)}$ ICTP, Trieste, Italy; ${ }^{(c)}$ Dipartimento di

Chimica, Fisica e Ambiente, Università di Udine, Udine, Italy

${ }^{166}$ Department of Physics, University of Illinois, Urbana, IL, USA

${ }^{167}$ Department of Physics and Astronomy, University of Uppsala, Uppsala, Sweden

${ }^{168}$ Instituto de Física Corpuscular (IFIC) and Departamento de Física Atómica, Molecular y Nuclear and Departamento de Ingeniería Electrónica and Instituto de Microelectrónica de Barcelona (IMB-CNM), University of Valencia and CSIC, Valencia, Spain

169 Department of Physics, University of British Columbia, Vancouver, BC, Canada

${ }^{170}$ Department of Physics and Astronomy, University of Victoria, Victoria, BC, Canada

171 Department of Physics, University of Warwick, Coventry, UK

172 Waseda University, Tokyo, Japan

${ }^{173}$ Department of Particle Physics, The Weizmann Institute of Science, Rehovot, Israel

174 Department of Physics, University of Wisconsin, Madison, WI, USA

175 Fakultät für Physik und Astronomie, Julius-Maximilians-Universität, Würzburg, Germany

${ }^{176}$ Fachbereich C Physik, Bergische Universität Wuppertal, Wuppertal, Germany

177 Department of Physics, Yale University, New Haven, CT, USA

178 Yerevan Physics Institute, Yerevan, Armenia

${ }^{179}$ Centre de Calcul de l'Institut National de Physique Nucléaire et de Physique des Particules (IN2P3), Villeurbanne, France

a Also at Department of Physics, King's College London, London, UK

${ }^{b}$ Also at Institute of Physics, Azerbaijan Academy of Sciences, Baku, Azerbaijan

${ }^{c}$ Also at Novosibirsk State University, Novosibirsk, Russia

${ }^{\mathrm{d}}$ Also at TRIUMF, Vancouver, BC, Canada

e Also at Department of Physics, California State University, Fresno, CA, USA

${ }^{\mathrm{f}}$ Also at Department of Physics, University of Fribourg, Fribourg, Switzerland

g Also at Departamento de Fisica e Astronomia, Faculdade de Ciencias, Universidade do Porto, Porto, Portugal

h Also at Tomsk State University, Tomsk, Russia

${ }^{i}$ Also at CPPM, Aix-Marseille Université and CNRS/IN2P3, Marseille, France

${ }^{\mathrm{j}}$ Also at Università di Napoli Parthenope, Naples, Italy

${ }^{\mathrm{k}}$ Also at Institute of Particle Physics (IPP), Victoria, Canada

${ }^{1}$ Also at Particle Physics Department, Rutherford Appleton Laboratory, Didcot, UK

${ }^{m}$ Also at Department of Physics, St. Petersburg State Polytechnical University, St. Petersburg, Russia

${ }^{\mathrm{n}}$ Also at Louisiana Tech University, Ruston, LA, USA

o Also at Institucio Catalana de Recerca i Estudis Avancats, ICREA, Barcelona, Spain

p Also at Department of Physics, National Tsing Hua University, Hsinchu, Taiwan

${ }^{q}$ Also at Department of Physics, The University of Texas at Austin, Austin, TX, USA 
${ }^{\mathrm{r}}$ Also at Institute of Theoretical Physics, Ilia State University, Tbilisi, Georgia

${ }^{\mathrm{s}}$ Also at CERN, Geneva, Switzerland

${ }^{\mathrm{t}}$ Also at Georgian Technical University (GTU), Tbilisi, Georgia

u Also at Ochadai Academic Production, Ochanomizu University, Tokyo, Japan

${ }^{v}$ Also at Manhattan College, New York, NY, USA

${ }^{w}$ Also at Institute of Physics, Academia Sinica, Taipei, Taiwan

${ }^{x}$ Also at LAL, Université Paris-Sud and CNRS/IN2P3, Orsay, France

y Also at Academia Sinica Grid Computing, Institute of Physics, Academia Sinica, Taipei, Taiwan

${ }^{\mathrm{z}}$ Also at School of Physics, Shandong University, Shandong, China

${ }^{\text {aa }}$ Also at Moscow Institute of Physics and Technology State University, Dolgoprudny, Russia

ab Also at Section de Physique, Université de Genève, Geneva, Switzerland

${ }^{a c}$ Also at International School for Advanced Studies (SISSA), Trieste, Italy

${ }^{\text {ad }}$ Also at Department of Physics and Astronomy, University of South Carolina, Columbia, SC, USA

${ }^{\text {ae }}$ Also at School of Physics and Engineering, Sun Yat-sen University, Guangzhou, China

${ }^{\text {af }}$ Also at Faculty of Physics, M.V. Lomonosov Moscow State University, Moscow, Russia

ag Also at National Research Nuclear University MEPhI, Moscow, Russia

ah Also at Department of Physics, Stanford University, Stanford, CA, USA

ai Also at Institute for Particle and Nuclear Physics, Wigner Research Centre for Physics, Budapest, Hungary

aj Also at Department of Physics, The University of Michigan, Ann Arbor, MI, USA

ak Also at Discipline of Physics, University of KwaZulu-Natal, Durban, South Africa

al Also at University of Malaya, Department of Physics, Kuala Lumpur, Malaysia

* Deceased 